\title{
W-algebras and superalgebras from constrained WZW models: a group theoretical classification.
}

\author{
L. Frappat因, E. Ragoucy* and P. Sorba*田 \\ Laboratoire de Physique Théorique ENSLAPP 目
}

\begin{abstract}
We present a classification of $W$ algebras and superalgebras arising in Abelian as well as non Abelian Toda theories. Each model, obtained from a constrained WZW action, is related with an $S l(2)$ subalgebra (resp. $O S p(1 \mid 2)$ superalgebra) of a simple Lie algebra (resp. superalgebra) $\mathcal{G}$. However, the determination of an $U(1)_{Y}$ factor, commuting with $S l(2)$ (resp. $O S p(1 \mid 2)$ ), appears, when it exists, particularly useful to characterize the corresponding $W$ algebra. The (super) conformal spin contents of each $W$ (super)algebra is performed. The class of all the superconformal algebras (i.e. with conformal spins $s \leq 2$ ) is easily obtained as a byproduct of our general results.

ENSLAPP-AL-391/92

July 1992

\footnotetext{
${ }^{*}$ Groupe d'Annecy, LAPP Chemin de Bellevue BP 110, F-74941 Annecy-le-Vieux Cedex, France

${ }^{\dagger}$ Groupe de Lyon, ENS Lyon 46 allée d'Italie, F-69364 Lyon Cedex 07, France

${ }^{\ddagger}$ URA 14-36 du CNRS, associée à l’E.N.S. de Lyon, et au L.A.P.P. (IN2P3-CNRS)
} 


\section{Contents}

1 Introduction 3

$2 W$ algebras and (half-)integral gradings 4

2.1 $W$ algebras in Toda theories ..............

2.2 Properties of (half) integral gradations . . . . . . . . . . . 7 ( . . . . . .

2.3 Primary fields of $W$ algebras . . . . . . . . . . . . . 8

2.4 Classification of constrained WZW models. . . . . . . . . . 9

I $W$ algebras built on Lie algebras

3 The different $S l(2)$ subalgebras in a simple Lie algebra $\mathcal{G}$

3.1 $S l(2)$ associated to regular subalgebras ............ 11

3.2 Exceptions . . . . . . . . . . . . . . . . . 12

3.3 Example: subalgebras of $S p(6) \ldots \ldots \ldots \ldots$

$4 S l(2)$ decompositions of simple Lie algebras 14

4.1 The $\mathcal{G}$ fundamental representation with respect to a $S l(2)$ subalgebra 14

4.1.1 $S l(n)$ case . . . . . . . . . . . 14

4.1.2 $S p(2 n)$ case ..................... 15

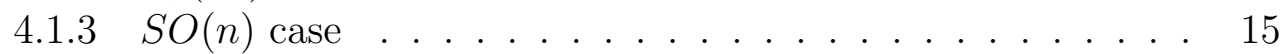

4.2 The $\mathcal{G}$ adjoint representation with respect to $S l(2)$ subalgebras . . 15

4.3 Example: case of $S O(8) \ldots \ldots \ldots \ldots$

$5 S l(2) \oplus U(1)_{Y}$ decompositions of simple Lie algebras 17

$5.1 S l(n)$ algebras .......................... 17

$5.2 S O(n)$ algebras $\ldots \ldots \ldots \ldots \ldots \ldots$

$5.3 \quad S p(2 n)$ algebras ..................... 18

5.4 The algebra $G_{2} \ldots \ldots \ldots \ldots \ldots$

5.5 The algebra $F_{4} \ldots \ldots \ldots \ldots \ldots$

6 Classification of (half-)integral gradings. 20

6.1 Defining vectors . . . . . . . . . . . . . . 20

6.2 Calculation of the defining vector from the $S l(2)$ decomposition . 21

6.3 Case of $S l(2) \oplus U(1)$ decomposition . . . . . . . . . . . 23

77 Poisson brackets of $W$ algebras 24

7.1 Generalities ..................... 24

7.2 Use of the stress-energy tensor . . . . . . . . . . . . . . 26

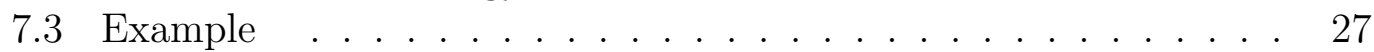

$8 W$ algebras from Lie algebras of rank up to 4 


\section{Super $W$ algebras built on Lie superalgebras}

9 The $O S p(1 \mid 2)$ subsuperalgebras of simple Lie superalgebras 37

$10 \operatorname{OSp}(1 \mid 2)$ decompositions of simple Lie superalgebras 38

10.1 The unitary superalgebras $S l(m \mid n) \ldots \ldots \ldots \ldots$

10.2 The orthosymplectic superalgebras $O S p(M \mid 2 n) \ldots \ldots \ldots \ldots$

10.2.1 Products of $O S p(1 \mid 2)$ irreducible representations . . . . 41

10.2 .2 The superalgebras $O S p(2 m \mid 2 n) \ldots \ldots \ldots \ldots \ldots$

10.2.3 The superalgebras $\operatorname{OSp}(2 m+1 \mid 2 n) \ldots \ldots \ldots \ldots$

10.2 .4 The irregular embeddings $\ldots \ldots \ldots \ldots \ldots$

10.2 .5 The superalgebras $O S p(2 \mid 2 n) \ldots \ldots \ldots \ldots \ldots$

10.3 Summary of the results $\ldots \ldots \ldots \ldots \ldots$

10.4 The exceptional superalgebra $\mathrm{G}(3) \ldots \ldots \ldots \ldots \ldots$

10.5 The exceptional superalgebra $\mathrm{F}(4) \ldots \ldots \ldots \ldots \ldots$

$11 \operatorname{OSp}(1 \mid 2) \oplus U(1)$ decompositions of simple Lie superalgebras 53

11.1 Introduction of the $U(1) \ldots \ldots \ldots \ldots$

11.2 Superdefining vector . . . . . . . . . . . . . 56

$12 W$ superalgebras from Lie superalgebras of rank up to 4

13 Quadratic-, Quasi- and $\mathbb{Z}_{2} \times \mathbb{Z}_{2^{-}}$Superconformal algebras 63

13.1 Quasi-superconformal algebras . . . . . . . . . . . . 63

13.2 Quadratic-superconformal algebras . . . . . . . . . . . 64

$13.3 \mathbb{Z}_{2} \times \mathbb{Z}_{2}$ superconformal algebras . . . . . . . . . 64

14. Conclusion 68 


\section{Introduction}

Lots of efforts have been done these recent years to detect and understand the infinite dimensional symmetries which underly two dimensional field theories. A particular role is played by Toda theories, since each of them possesses a $W$ symmetry [1, 2]. More recently, it has been shown that in fact Toda models can be seen as constrained WZW models [3]. One can say that such a property reenforces the fundamental role of WZW models in the realm of conformal field theories. It also provides a natural framework to compute explicitly the $W$ algebras which then appear.

In order to reduce a WZW model to a Toda one, some of the conserved current components have to be set to constants or zero. It can be realized that, from a given simple Lie algebra (or superalgebra) $\mathcal{G}$, different choices of constraints can be proposed, each of them giving rise to a different Toda model, to which will be associated a $W$ (super)algebra. Actually, to each such a Toda model corresponds

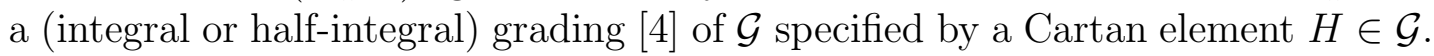
In other words, $\mathcal{G}$, which is chosen maximally non-compact, admits a vector space decomposition:

$$
\mathcal{G}=\bigoplus_{h \in \frac{1}{2} \mathbf{Z}} \mathcal{G}_{h} \quad \text { with } \quad\left[H, X_{h}\right]=h X_{h} \quad \text { for any } \quad X_{h} \in \mathcal{G}_{h}
$$

As an example, the usual or Abelian Toda model associated to $\mathcal{G}$ is obtained by taking $H$ as the Cartan generator of the principal $S l(2)$ in the algebra (or superprincipal $O S p(1 \mid 2)$ in the superalgebra) $\mathcal{G}$.

For each such a grading $H$ can be defined either an $S l(2)$ [4, 5, 6, 7] or an $S l(2) \oplus U(1)$ [8] (resp. $O S p(1 \mid 2)$ [9, 10] or $O S p(1 \mid 2) \oplus U(1))$ sub(super)algebra of $\mathcal{G}$ generated by $\left\{M_{0}, M_{ \pm}\right\} \oplus\{Y\}$ (resp. $\left.\left\{M_{0}, M_{ \pm}, F_{ \pm}\right\} \oplus\{Y\}\right)$ and such that $H=M_{0}+Y$. More precisely, even when the $U(1)$ part is not zero, the $S l(2)$ (resp. $O S p(1 \mid 2))$ subalgebra is sufficient to characterize the $W$ algebra: one can then say that the different Toda models in $\mathcal{G}$ are classified by the different $S l(2)$ (resp. $O S p(1 \mid 2))$ subalgebras of $\mathcal{G}$. However, interesting informations on the structure of the corresponding $W$ algebra can be obtained when the $Y$ generator exists. As will be shown below, a conserved hypercharge can be associated to it, which may greatly simplify the Poisson bracket computation of the different primary fields constituting the $W$ algebra.

The determination of the semi-simple subalgebras of a simple Lie algebra has been considered by Dynkin [11] and explicited for algebras of rank up to 6 by Lorente and Gruber [12]. Hereafter, we have reconsidered in some details this classification in order to study the $S l(2)$ and $S l(2) \oplus U(1)$ subalgebras. The reduction of the fundamental and adjoint representations of a (semi-)simple Lie 
algebra is achieved in the general case, allowing to determine, by working in the so-called highest weight Drinfeld-Sokolov gauge [13, 3], the conformal spin content of the $W$ algebras. Tables relative to algebras of low rank $(r \leq 4)$ are provided. The usefulness of the conserved hypercharge $Y$ is illustrated to calculate the $\mathrm{PB}$ of the algebra of spins $2, \frac{3}{2}, \frac{3}{2}, 1$ first considered in [14, 15].

As already mentioned, in the supersymmetric case when $\mathcal{G}$ is a simple Lie superalgebra, the $S l(2)$ algebra is replaced by its supersymmetric "extension" $O S p(1 \mid 2)$ 9, 10]. It is therefore the classification of $O S p(1 \mid 2) \oplus U(1)$ subsuperalgebras in $\mathcal{G}$ which is now of interest. The perfect knowledge of the algebra case helps a lot for this supersymmetric generalization. Note that a first attempt in this direction can be found in [9]. Hereafter, we explicitly achieve this classification in a way which, we believe, is clear and allows a direct use. As in the algebra case, general formulae for the decomposition of the fundamental and adjoint representations of a simple Lie superalgebra with respect to $O S p(1 \mid 2) \oplus U(1)$ subsuperalgebras are given, and the (super)conformal spin content of the super $W$ algebras determined. In order to illustrate these results, and mainly to allow a comparison with the extended superconformal algebras [16], tables are constructed for superalgebras of rank up to 4 .

\section{$2 \quad W$ algebras and (half-)integral gradings}

\section{1 $W$ algebras in Toda theories}

It has been elegantly shown that, starting from a WZW model, the action of which is $S(g)$ and the fields $g(x)$ (resp. superfields $g(x, \theta)$ ) belong to the group (resp. supergroup) $G$, and imposing some of the components of the conserved (super) currents to be constant or zero leads to a Toda model.

Let us, at this point, briefly fix some notations.

As far as $G$ is a group, the WZW conserved currents read:

$$
J_{+}=g^{-1} \partial_{+} g \quad J_{-}=\left(\partial_{-} g\right) g^{-1}
$$

with

$$
\partial_{-} J_{+}=\partial_{+} J_{-}=0
$$

When considering a supersymmetric WZW model [10], a supergroup element will locally be defined as:

$$
g(x, \theta)=\exp \left(\varphi^{i} B_{i}+\psi^{j} F_{j}\right)
$$


where the $\varphi^{i}$ (resp. $\psi^{j}$ ) are bosonic (resp. fermionic) superfields, and the $B_{i}$ (resp. $F_{j}$ ) commuting (resp. anticommuting) generators in the considered finite dimensional superalgebra $\mathcal{G}$. Then the corresponding supercurrents are:

$$
J_{+}=\hat{g}^{-1} D_{+} g \quad J_{-}=\left(D_{-} g\right) g^{-1}
$$

where $\hat{g}$ differs from $g$ by the change of sign on its fermionic generator part, the bosonic ones staying unchanged. We note that the fermionic character of $D_{ \pm}=\theta_{ \pm} \partial_{x_{ \pm}}+\partial_{\theta_{ \pm}}$implies the supercurrents to develop as:

$$
J=\Psi^{i} B_{i}+\Phi^{j} F_{j}
$$

the $\Psi^{i}$ being fermionic and the $\Phi^{j}$ bosonic superfields.

The choice of the $J$ components which are constrained to be constant with respect to those which are put to zero naturally defines a grading (see 1.1) on the (super)algebra $\mathcal{G}$. The simplest and most known example is the Abelian Toda model relative to $\mathcal{G}$. In this case the $\mathrm{J}$ components associated to the opposite of the simple roots have constant values while those relative to the other negative roots are put to zero. The grading is ruled by the generator $H$, sum of the Cartan generators in the Cartan Weyl basis. The $\mathcal{G}$ subalgebra $\mathcal{G}_{0}$ is exactly the Cartan subalgebra of $\mathcal{G}$ in this basis, the simple root generators $E_{+\alpha}$ form the $\mathcal{G}$ subspace $\mathcal{G}_{+1}$, and their partners $E_{-\alpha}$ the subspace $\mathcal{G}_{-1}$; finally $\mathcal{G}_{+}$is constructed from the positive roots and $\mathcal{G}_{-}$from the negative ones.

As could be expected, imposing a set of constraints reduces the huge symmetry provided by the Kac-Moody current algebra to a subset of quantities, polynomials in the current components and their derivatives, which will constitute a $W$-algebra. For example, the original conformal symmetry of the WZW model itself is broken when constraints corresponding to the grading $H$ are imposed, and in order to construct the Virasoro symmetry for this Toda model a $H$ dependent correction term has to be added to the former one.

More precisely, the stress energy tensor reads [3]:

$$
T_{H}=\frac{1}{2} \operatorname{Tr} J^{2}-\operatorname{Tr} H \partial J
$$

when $\mathcal{G}$ is an algebra, and [10]:

$$
T_{H}=S t r\left(\frac{1}{3} J \hat{J} J+\frac{1}{2} J D J\right)-S \operatorname{tr}\left(H \cdot D^{2} J\right)
$$

when $\mathcal{G}$ is a superalgebra.

The determination of the other generators of the $W$ algebra can be achieved as follows. 
If $\mathcal{G}$ is an algebra, one selects in $\mathcal{G}_{-1}$ a (constant) element $M_{-}$such that 3

$$
\operatorname{Ker}\left(\operatorname{ad} M_{-}\right) \cap \mathcal{G}_{+}=\{0\} .
$$

Then one expresses $J$ as:

$$
J=M_{-}+J_{>-1}
$$

where the variable dependent part $J_{>-1}$ belongs to $\oplus_{h} \mathcal{G}_{h}$ with $h>-1$.

If $\mathcal{G}$ is a superalgebra, then one picks up in $\mathcal{G}_{-1 / 2}$ a fermionic (constant) element $F_{-}$with $\left\{F_{-}, F_{-}\right\}=M_{-} \neq 0$ such that:

$$
\operatorname{Ker}\left(a d F_{-}\right) \cap \mathcal{G}_{+}=\{0\}
$$

and one expresses $J$ as:

$$
J=F_{-}+J_{>-\frac{1}{2}}
$$

Finally one has just to use the gauge transformations:

$$
J \rightarrow g J g^{-1}+(\partial g) g^{-1}
$$

where $g$ belongs to the local Lie groups generated by $\mathcal{G}_{+}$, or:

$$
J \rightarrow \hat{g} J g^{-1}+\left(D_{-} g\right) g^{-1}
$$

in the supersymmetric case, to transform $J$ into:

$$
J^{\prime}=\mu_{-}+\sum_{h} W_{h+1}(J) X_{h} \quad \text { with } \quad \mu_{-}=M_{-}\left(\operatorname{resp} . F_{-}\right)
$$

where the $W_{h+1}(J)$ are gauge invariant polynomials generating the $W$ algebra associated to the Toda theory. The PB among $W$ generators will be calculated from the PB:

$$
\left\{J^{a}(x), J^{b}\left(y^{\prime}\right)\right\}_{P B}=i f_{c}^{a b} \delta\left(x-x^{\prime}\right) J^{c}\left(x^{\prime}\right)+k^{a b} \partial_{x} \delta\left(x-x^{\prime}\right) .
$$

when $\mathcal{G}$ is a Lie algebra and:

$$
\left\{J^{a}(X), J^{b}\left(X^{\prime}\right)\right\}_{P B}=i(-1)^{[a](1+[b])} f_{c}^{a b} \delta\left(X-X^{\prime}\right) J^{c}\left(X^{\prime}\right)+k \eta^{a b} D_{x} \delta\left(X-X^{\prime}\right) .
$$

when $\mathcal{G}$ is a superalgebra. $f_{c}^{a b}$ are the structure constants, $\eta^{a b}$ the scalar product and $k$ the central extension parameter of the Kac Moody (super)algebra; by $[a]$ is expressed the $\mathbb{Z}_{2}$ grading of the generator $T^{a}:[a]=0$ (resp. 1) if $T^{a}$ is a commuting (resp. anticommuting) generator (see [10 for more details).

Using (2.7) one understands that $W_{h+1}(J)$ has a (super) conformal weight $1+h$ under $T_{H}$. 
Note that the condition (2.8) expresses the non degeneracy for $h>0$, of the operator:

$$
a d M_{-}: \mathcal{G}_{h} \rightarrow \mathcal{G}_{h-1} .
$$

Then Drinfeld-Sokolov (D.S.) gauges can be used to determine a complete set of gauge invariant quantities $W_{h+1}(J)$. In the highest weight D.S. gauge, each $W_{h+1}(J)$ is "carried" by the highest weight of a given $S l(2)$ subalgebra built from $M_{-}$.

\subsection{Properties of (half) integral gradations}

We have presented in [8] three propositions establishing a correspondence between (integral and half integral) gradings of a simple Lie algebra $\mathcal{G}$ which specify Toda theories, and $S l(2) \oplus U(1)$ subalgebras of $\mathcal{G}$. The generalisation to the superalgebra case is straightforward, replacing the $S l(2)$ part by its "supersymmetric extension" $O S p(1 \mid 2)$. Therefore, we limit ourselves to enounce hereafter these properties.

Let $H$ be a grading operator of a (super)algebra $\mathcal{G}$. Then:

Proposition 1:

i) $\mathcal{G}$ being an algebra, any element $M_{-} \in \mathcal{G}_{-}$can be embedded in one of its $S l(2)$ subalgebra.

ii) $\mathcal{G}$ being a superalgebra, any fermionic element $F_{-} \in \mathcal{G}_{-}$with $\left\{F_{-}, F_{-}\right\}=$ $M_{-} \neq 0$ can be embedded in one of its $O S p(1 \mid 2)$ subalgebra.

Proposition 2:

$\overline{\text { Let } M_{-} \in \mathcal{G}_{-1}}$ (resp. $\left.F_{-} \in \mathcal{G}_{-1 / 2}\right)$. Then, it is always possible to write $H$ as:

$$
H=M_{0}+Y
$$

with $M_{0}$ being the Cartan part of an $S l(2)$ algebra constructed from $M_{-}$(resp. an $O S p(1 \mid 2)$ superalgebra built on $\left.F_{-}\right)$, and the generator $Y$ commuting, when non zero, with this three (resp. five) dimensional subalgebra.

Moreover, the $S l(2)$ part constructed from $M_{-}$(resp. $O S p(1 \mid 2)$ superalgebra built on $F_{-}$) is unique up to a conjugation by group elements generated from the subalgebra $\hat{\mathcal{G}}_{0}=\operatorname{Ker}\left(a d M_{-}\right) \cap \mathcal{G}_{0}$.

Proposition 3:

i) Let $M_{-}, M_{0}, M_{+}$and $Y$ generate an $S l(2) \oplus U(1)$ subalgebra of $\mathcal{G}$ with $M_{-} \in \mathcal{G}_{-1}$ and $M_{0}+Y=H$. Decompose $\mathcal{G}$, considered as a vector space, into 
irreducible representations $\mathcal{D}_{j_{i}}\left(y_{i}\right)$ of this algebra, where $y_{i}$ denotes the eigenvalue of $Y$ on the $S l(2)$ representation $\mathcal{D}_{j_{i}}$. Then

$$
\operatorname{Ker}\left(a d M_{-}\right) \cap \mathcal{G}_{+}=\{0\} \quad \text { iff } \quad\left|y_{i}\right| \leq j_{i} \quad \text { for any } \quad \mathcal{D}_{j_{i}}\left(y_{i}\right) \quad \text { in } \quad \mathcal{G} .
$$

ii) Let $M_{-}, F_{-}, M_{0}, F_{+}, M_{+}$and $Y$ generate an $O S p(1 \mid 2) \oplus U(1)$ subsuperalgebra of $\mathcal{G}$ with $F_{-} \in \mathcal{G}_{-1 / 2}$ and $M_{0}+Y=H$. Decompose $\mathcal{G}$, considered as a vector space, into irreducible representations $\mathcal{R}_{j_{i}}\left(y_{i}\right)$ of this algebra, where $y_{i}$ denotes the eigenvalue of $Y$ on the $O S p(1 \mid 2)$ representation $\mathcal{R}_{j_{i}}=\mathcal{D}_{j_{i}} \oplus \mathcal{D}_{j_{i}-1 / 2}$. Then

$$
\operatorname{Ker}\left(a d F_{-}\right) \cap \mathcal{G}_{+}=\{0\} \quad \text { iff } \quad\left|y_{i}\right| \leq j_{i} \quad \text { for any } \quad \mathcal{R}_{j_{i}}\left(y_{i}\right) \quad \text { in } \quad \mathcal{G} .
$$

in the following, we will call the condition (2.19) (resp. 2.20) a non degeneracy condition for $a d M_{-}$(resp. for $\left.a d F_{-}\right)$. Of course, as the grades satisfy $h_{i}=j_{i}+y_{i}$, one must impose $h_{i} \in \frac{1}{2} \mathbb{Z}$ in the $\mathcal{G}$ adjoint representation to have (half)integral grading.

These three propositions have to be completed by:

Proposition 4:

The gradations $H=M_{0}+Y$ and $M_{0}$ lead to the same $W$ algebra.

This last proposition has been proven in [7]. From the point of view of the decomposition under $S l(2) \oplus U(1)$, note that (2.19) ensures that the highest weight of the $S l(2)$ subalgebra are in the $\mathcal{G}_{\geq 0}$ part of $\mathcal{G}$ for both $H=M_{0}$ and $H=M_{0}+Y$ gradations. This is in agreement with the "halving" used in [7].

\subsection{Primary fields of $W$ algebras}

The spin of the $W$ generators corresponding to a given gradation $H$ are obtained from the highest weights of the $S l(2) \oplus U(1)$ (resp. $O S p(1 \mid 2) \oplus U(1)$ ) decomposition of the $\mathcal{G}$-adjoint representation (DS gauge). Now, we have to know whether the $W$ generators are (super) primary fields under $T_{H}$. The (super) primary fields satisfy the following Poisson bracket:

$$
\begin{aligned}
\left\{T_{H}(x), W_{h+1}\left(x^{\prime}\right)\right\}_{P B}= & (h+1) W_{h+1}\left(x^{\prime}\right) \partial_{x} \delta\left(x-x^{\prime}\right)+\partial W_{h+1}\left(x^{\prime}\right) \delta\left(x-x^{\prime}\right) \\
\left\{T_{H}(X), W_{h+1 / 2}\left(X^{\prime}\right)\right\}_{P B}= & \left(h+\frac{1}{2}\right) \partial_{x} \delta\left(X-X^{\prime}\right) W_{h+1}\left(X^{\prime}\right)+\delta\left(X-X^{\prime}\right) \partial W_{h+1}\left(X^{\prime}\right)+ \\
& -\frac{1}{2} D_{X} \delta\left(X-X^{\prime}\right) W_{h+1 / 2}\left(X^{\prime}\right)
\end{aligned}
$$

where we have used for the supersymmetric case the conventions

$$
X=(x, \theta) \quad \text { and } \quad \delta\left(X-X^{\prime}\right)=\left(\theta-\theta^{\prime}\right) \delta\left(x-x^{\prime}\right)
$$


Note that (2.21) corresponds to PB between fields and (2.22) between superfields. We will say, in the former case, that $W_{h+1}$ has spin $h+1$, whereas, in the latter case, $W_{h+1 / 2}$ carries a superspin $h+\frac{1}{2}$. In fact, it is clear from the expression of $T_{H}$ that the only generators $W_{h+1}$ (resp. $W_{h+1 / 2}$ ) which are not primary are those which satisfy $<H, X_{h}>\neq 0$, where $<,>$ is the $\mathcal{G}$ non degenerated scalar product and $X_{h}$ is the generator of $\mathcal{G}$ carrying $W_{h+1}$ (resp. $W_{h+1 / 2}$ ) in (2.14). This implies that $X_{h}$ is a Cartan generator, so that $h=0$ and $W_{h+1} \equiv W_{1}$ (resp. $W_{h+1 / 2} \equiv W_{1 / 2}$ ) forms a singlet representation of $S l(2)$ (resp. $O S p(1 \mid 2)$ ). Actually, by linear combinations, one can always eliminate these non primary generators, but one. Since for $H=M_{0}$ all the $W$ generators are primary (except $T_{M_{0}}$ of course), we can think at the non primary generator as carried by $Y$ itself [7]. This is ensured by the equality

$$
<H, Y>=<M_{0}+Y, Y>=<Y, Y>\neq 0 \quad \text { iff } \quad Y \neq 0
$$

We will call this (super) generator $W_{1}^{Y}$ (resp. $W_{1 / 2}^{Y}$ ). Note that because of its spin 1 (resp. superspin $\frac{1}{2}$ ), the $\mathrm{PB}$ of $T_{H}$ with $W_{1}^{Y}\left(W_{1 / 2}^{Y}\right)$ differs from the $\mathrm{PB}$ of $T_{H}$ with a (super) primary field only by a central extension term, corresponding to a second order derivative (resp. fermionic derivative $D$ ) of a (super)delta distribution.

Thus, all the $W$ generators are primary with respect to $T_{H}$, except $T_{H}$ itself and, when $Y \neq 0$, a spin 1 generator $W_{1}^{Y}$ (resp. a superspin $\frac{1}{2}$ generator $W_{1 / 2}^{Y}$ ) carried by $Y$. In that case, $W_{1}^{Y}\left(W_{1 / 2}^{Y}\right)$ differs from a primary field (resp. superfield) by a central extension term.

\subsection{Classification of constrained WZW models.}

The above properties suggest a way to determine all the different (super)Toda models associated with (half-)integral gradings of a simple Lie (super)algebra $\mathcal{G}$, and their corresponding (super) $W$ algebras, namely:

i) Classify all the $S l(2)$ (resp. $O S p(1 \mid 2))$ sub(super)algebras of $\mathcal{G}$

ii) Add to each of these simple sub(super)algebras a commuting $U(1)$ factor such that in the decomposition of the $\mathcal{G}$ adjoint representation into $S l(2) \oplus U(1)$ representations $\mathcal{D}_{j_{i}}\left(y_{i}\right)$ (resp. $O S p(1 \mid 2) \oplus U(1)$ representations $\mathcal{R}_{j_{i}}\left(y_{i}\right)$ ), the following conditions hold:

$$
\begin{aligned}
& \left|y_{i}\right| \leq j_{i} \quad i=1, \cdots, n \\
& j_{i}+y_{i} \in \mathbb{Z} \quad \text { (integral grading) } \quad j_{i}+y_{i} \in \frac{1}{2} \mathbb{Z} \quad \text { (half-integral grading(g)26) }
\end{aligned}
$$

\footnotetext{
${ }^{1}$ Note that the two components of the superfield $W_{h+1 / 2}$ are of conformal spins $h+\frac{1}{2}$ and $h+1$.
} 
Note that the $y_{i}$ values are naturally restricted when calculating the $S l(2) \oplus$ $U(1)$ (resp. $O S p(1 \mid 2) \oplus U(1))$ decomposition of the adjoint representation of $\mathcal{G}$ coming from the product of fundamental representations already decomposed into $S l(2) \oplus U(1)$ (resp. $O S p(1 \mid 2) \oplus U(1)$ ) representations: this will be explicited in the following.

iii) Then to each such an $S l(2) \oplus U(1)$ (resp. $O S p(1 \mid 2) \oplus U(1))$ sub(super)algebra of $\mathcal{G}$ satisfying (2.25) and (2.26) there will correspond a classical (i.e. PB) $W$ algebra generated by the $n$ elements $W_{h_{1}+1}, \ldots, W_{h_{n}+1}\left(\operatorname{resp} . W_{h_{1}+1 / 2}, \ldots, W_{h_{n}+1 / 2}\right)$ of conformal (super)spin under the (super)Virasoro algebra defined in (2.6, 2.7) $h_{1}+1, \ldots, h_{n}+1$ (resp. $\left.h_{1}+\frac{1}{2}, \ldots, h_{n}+\frac{1}{2}\right)$ with $h_{i}$ given by

$$
h_{i}=y_{i}+j_{i}
$$

as a consequence of a Drinfeld-Sokolov highest weight gauge [3, 13].

$i v)$ Reconstruct the grading $H$ from the $S l(2) \oplus U(1)$ (resp. $O S p(1 \mid 2) \oplus U(1)$ ) decomposition. Varying $Y$ for a fixed $S l(2)(O S p(1 \mid 2)$ super)algebra will give all the isomorphic gradations.

v) Deduce informations of the $\mathrm{PB}$ from the $S l(2) \oplus U(1)$ (resp. $O S p(1 \mid 2) \oplus$ $U(1)$ ) reduction.

These five steps will be explicited in the following. In the part $\mathbb{I}$, we will focus on the algebras case, while in the part $\mathbb{1}$ the previous results will be used to state the superalgebras case. 


\section{Part I}

\section{$W$ algebras built on Lie algebras}

\section{The different $S l(2)$ subalgebras in a simple Lie algebra $\mathcal{G}$}

\section{1 $S l(2)$ associated to regular subalgebras}

The classification of $S l(2)$ subalgebras of a simple Lie algebra $\mathcal{G}$ has been achieved by Dynkin [11]. His technics can be summarized as follows:

Any $S l(2)$ subalgebra in $\mathcal{G}$ can be seen, up to a few exceptions occuring in $D_{n}$ and $E_{6,7,8}$ algebras (which will be discussed in a moment), as the principal $S l(2)$ algebra of a regular $\mathcal{G}$ subalgebra.

Let us remind that the principal $S l(2)$ subalgebra of a (semi) simple algebra $\mathcal{G}$ admits as a positive vector root, the generator:

$$
E_{+}=\sum_{\alpha \in \Phi_{+}} E_{\alpha}
$$

if $\Phi_{+}$denotes a system of simple roots in $\mathcal{G}$.

In order to define a regular subalgebra of $\mathcal{G}$, let us consider, once given a Cartan subalgebra $\mathcal{H}$, the canonical vector space decomposition:

$$
\mathcal{G}=\left(\bigoplus_{\alpha \in \Delta} \mathcal{G}_{\alpha}\right) \oplus \mathcal{H}
$$

where $\Delta$ is the root system of $\mathcal{G}$, and $\mathcal{G}_{\alpha}$ the one dimensional $\mathcal{G}$ subspace associated to the root $\alpha$. Then, a subalgebra $\mathcal{G}^{\prime}$ of $\mathcal{G}$ is said regular if it can be seen, up to a conjugation of $\mathcal{G}$, as:

$$
\mathcal{G}^{\prime}=\left(\bigoplus_{\beta \in \Delta^{\prime}} \mathcal{G}_{\beta}\right) \oplus \mathcal{H}^{\prime}
$$

with $\Delta^{\prime} \subset \Delta$ and $\mathcal{H}^{\prime} \subset \mathcal{H}$. Obviously, the principal $S l(2)$ of $\mathcal{G}$ is not regular (except if $\mathcal{G}=S l(2)$ ). When a subalgebra is not regular, it is said singular.

Discarding the possible $U(1)$ factors, one can assert that a semi-simple regular subalgebra of $\mathcal{G}$ admits as Dynkin diagram (DD) a subdiagram of the DD of $\mathcal{G}$. By subdiagram of $\mathcal{G}$, we understand a DD obtained in the following way. 
i) First extend the $\mathcal{G}-\mathrm{DD}$ by adjunction of the lowest root of $\mathcal{G}$, and consider the different DD's obtained by removing one dot.

ii) For these diagrams repeat the procedure $i$ ) to each of their connected parts until no new (connected or not connected) diagram with $r=\operatorname{rank}(\mathcal{G})$ dots is obtained.

iii) Finally, from each such a "maximal" subdiagram, remove $m$ dots with $0 \leq m<r$. One gets in this way all the $\mathcal{G}$ subdiagrams.

Of course, by this construction, the same subdiagram may appear several times. Such identical diagrams correspond in general to conjugate subalgebras, with three exceptions as long as we discard the $E$ series: see below.

When the $\mathcal{G}-\mathrm{DD}$ possesses roots of different length (the long ones represented by $\bigcirc$, and the short ones by $\bigcirc$ ) it is understood (and rather obvious) that the two subdiagrams $\bigcirc$ and correspond to two non-conjugate $S l(2)$ algebras.

\subsection{Exceptions}

We have explicited in section 3.1 how to get the regular subalgebras of a given algebra $\mathcal{G}$ using the method of subdiagrams. In that way, we get most of the $S l(2)$ embeddings of $\mathcal{G}$, the other ones being obtained as follows:

1) Regular subalgebras of the type $A_{n_{1}}+A_{n_{2}}+\cdots+A_{n_{k}}$, with $n_{1}+n_{2}+\cdots+n_{k}=$ $2 n-k$ and $n_{i}$ 's all odd, show up twice in $D_{2 n}, n>2$ (three times in $D_{4}$ ), each of them being related to the other by an outer automorphism of $D_{2 n}$. As an example, there are two regular subalgebras of the type $3 A_{1}, A_{1}+A_{3}$ and $A_{5}$ in $D_{6}$.

2) For $\mathcal{G}=B_{n}$ and $D_{n}, n>3$, the diagram $\bigcirc-\bigcirc$ must be considered twice, one been related to an "algebra $A_{3}$ ", and the other one to " $D_{3}$ ". Indeed, the $\mathcal{G}$ subdiagram

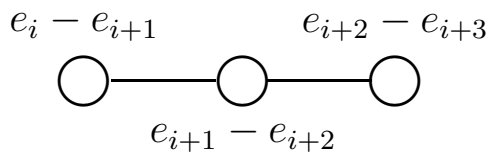

defines a system of simple roots for " $A_{3}$ ", while the subdiagram

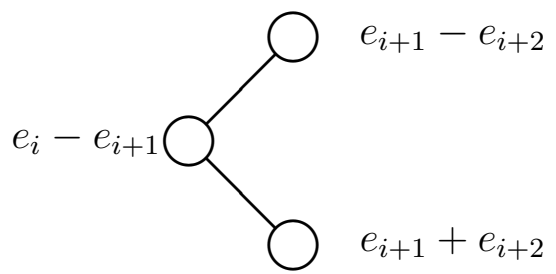


provides a system of simple roots of " $D_{3}$ ". In order to convince the reader, we remark that the fundamental representation of $D_{n}$ reduces with respect to $A_{3}$ as $\underline{2 n}=\underline{4}+\overline{4}+(2 n-8) \underline{1}$, and with respect to $D_{3}$ as $\underline{2 n}=\underline{6}+(2 n-6) \underline{1}$.

3) Again $B_{n}$ and $D_{n}$ admit two different types of $2 A_{1}$ subalgebras associated to the diagrams

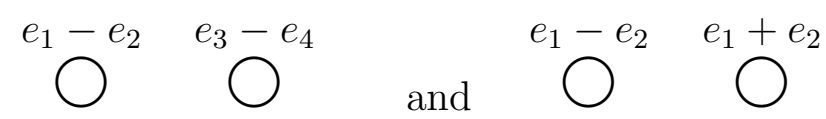

The fundamental of $D_{n}$ reduces with respect to the first algebra as $\underline{2 n}=$ $(\underline{2}+\overline{2}, \underline{0})+(\underline{0}, \underline{2}+\overline{2})+(2 n-8)(\underline{0}, \underline{0})$ and with respect to the second as $\underline{2 n}=(\underline{2}, \underline{2})+$ $(2 n-4)(\underline{0}, \underline{0})$. We can note that as well as in case 1$)$, it is the bifurcation appearing in the (extended) DD of $\left(B_{n}\right) D_{n}$ which is responsible for these doublings.

4) As mentioned at the beginning of the previous paragraph, there exist also $S l(2)$ subalgebras which cannot be seen as principal $S l(2)$ part of a regular subalgebra. This concerns only the $D_{n}$ and $E_{6,7,8}$ algebras. In the $D_{n}$ case, one has to add $\left[\frac{n-2}{2}\right] S l(2)$ subalgebras, each of them being a principal subalgebra of the singular ones:

$$
B_{i} \oplus B_{j} \quad \text { with } \quad i+j=n-1 \quad \text { and } \quad i \neq j
$$

For example, in $D_{4} \equiv S O(8)$ and $D_{5} \equiv S O(10)$, there exists one algebra of this type, associated to $S O(3) \oplus S O(5)$ and $S O(3) \oplus S O(7)$ respectively. In $D_{6} \equiv S O(12)$, two new $S l(2)$ algebras, principal with respect to $S O(3) \oplus S O(9)$ and $S O(5) \oplus S O(7)$ have to be considered.

\subsection{Example: subalgebras of $S p(6)$}

We illustrate the method on the algebral $C_{3}$. Its DD and extended DD are respectively:
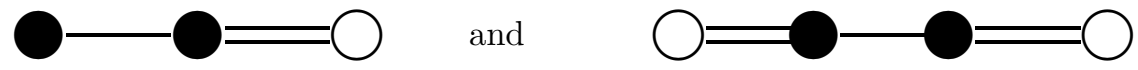

Deleting one dot, we get at the first step:
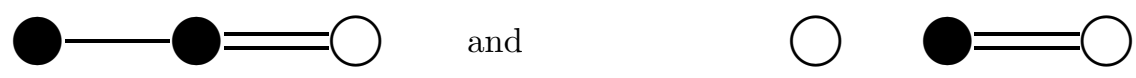

\footnotetext{
${ }^{2}$ We remind the notations: $A_{n} \equiv S l(n+1), B_{n} \equiv S O(2 n+1), C_{n} \equiv S p(2 n)$ and $D_{n} \equiv$ $S O(2 n)$.
} 
which correspond to the subalgebras $C_{3}$ and $A_{1} \oplus C_{2}$ respectively. Then, extending once more this last diagram, we get a new three dots DD:

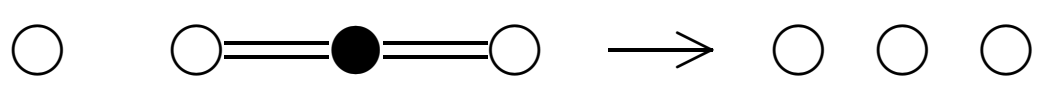

There is no new possibility to get three dots DD by the extension-deletion procedure. A direct chirurgy on them leads to the DD:

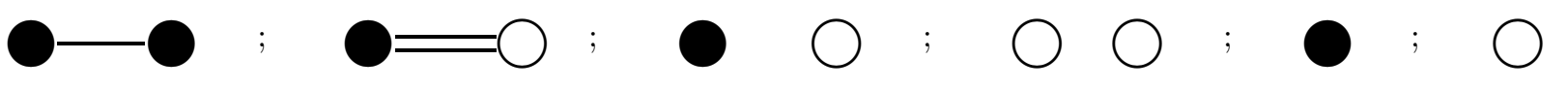

Gathering all these DD's, we can list the regular (semi-)simple subalgebras of $C_{3}$ :

$$
\begin{array}{ll}
\text { Rank } 3 \text { subalgebras } & C_{3} ; C_{2} \oplus A_{1} ; 3 A_{1} \\
\text { Rank } 2 \text { subalgebras } & C_{2} ; A_{2}^{2} ; 2 A_{1} ; A_{1} \oplus A_{1}^{2} \\
\text { Rank } 1 \text { subalgebras } & A_{1} ; A_{1}^{2}
\end{array}
$$

where the $S l(2)$ and $S l(3)$ subalgebras built on short roots are marked with the superscript (Dynkin index) 2. To each regular subalgebra of $C_{3}$ will be associated a principal $S l(2)$ subalgebra, and this construction will exhaust the set of $S l(2)$ subalgebras.

\section{$4 \quad S l(2)$ decompositions of simple Lie algebras}

Given any $S l(2)$ subalgebra of a Lie algebra $\mathcal{G}$ in the $A, B, C, D$ series, we need to know the decomposition of the adjoint representation of $\mathcal{G}$ with respect to this three dimensional subalgebra. For such a purpose, we will first compute the $S l(2)$ decomposition of the $(1,0,0, \ldots, 0)$ fundamental representation of $\mathcal{G}$. We will deduce the $S l(2)$ decomposition of the $\mathcal{G}$ adjoint representation by computing the product of the fundamental representation by its contragredient one: for the $A_{n}$ series, the adjoint representation is given by this product, once throwing away a trivial representation, while in the $B_{n}$ and $D_{n}$ (resp. $C_{n}$ ) cases, one has to select the antisymmetric (resp. symmetric) part.

\subsection{The $\mathcal{G}$ fundamental representation with respect to a $S l(2)$ subalgebra}

\subsection{1 $S l(n)$ case}

Any $S l(2)$ subalgebra is the principal subalgebra of a (sum of) $S l(p)$ subalgebra(s) in $S l(n)$. For each $S l(p)$ subalgebra will correspond a $\mathcal{D}_{(p-1) / 2}$ representa- 
tion of $S l(2)$ in the $\underline{n}$ of $S l(n)$, which will be completed with singlets.

For instance, if we look at the $S l(2)$ principal subalgebra of $S l(p) \oplus S l(q)$ in $S l(n)$, we will have

$$
\underline{n}=\mathcal{D}_{(p-1) / 2} \oplus \mathcal{D}_{(q-1) / 2} \oplus(n-p-q) \mathcal{D}_{0}
$$

\subsection{2 $S p(2 n)$ case}

An $S l(2)$ subalgebra is the principal subalgebra of a (sum of) $S p(2 p)$ subalgebra(s), $S l(2)^{1}$ subalgebra(s), or $S l(q)^{2}$ subalgebra(s). The superscript refers to the Dynkin index of the $S l(m)$ subalgebra considered: it is 1 when the $S l(2)$ subalgebra is constructed on a long root, and 2 in the other cases. The $S p(2 p)$ subalgebra contributes to the fundamental representation via a $\mathcal{D}_{p-(1 / 2)} S l(2)$ representation, while the $S l(q)^{2}$ (resp. $\left.S l(2)^{1}\right)$ yields the $\mathcal{D}_{(q-1) / 2}+\overline{\mathcal{D}}_{(q-1) / 2}$ (resp. $\left.\mathcal{D}_{1 / 2}\right)$ representations. The $\underline{2 n}$ representation is then completed by singlets. For example, for the decomposition of $S p(2 n)$ under the principal $S l(2)$ of $S p(2 p) \oplus S l(q)^{2} \oplus r S l(2)^{1}$, we have:

$$
\underline{2 n}=\mathcal{D}_{p-(1 / 2)} \oplus\left(\mathcal{D}_{(q-1) / 2} \oplus \overline{\mathcal{D}}_{(q-1) / 2}\right) \oplus r \mathcal{D}_{1 / 2} \oplus(2 n-2 p-2 q-2 r) \mathcal{D}_{0}
$$

\subsection{3 $S O(n)$ case}

When $S l(2)$ is principal subalgebra of either an $S O(2 p+1)$ or an $S O(2 p+2)$ one, the $\underline{n}$ fundamental of $S O(n)$ contains a $\mathcal{D}_{p}$ representation. In the case of an $S l(q), q \neq 2$, subalgebra, then it is the sum $\mathcal{D}_{(q-1) / 2} \oplus \overline{\mathcal{D}}_{(q-1) / 2}$ which shows up. For $q=2$, one must distinguish the case $S l(2)^{1}$ (long root) which leads to $\mathcal{D}_{1 / 2} \oplus \overline{\mathcal{D}}_{1 / 2}$ from the case $S l(2)^{2}$ (short root) leading to $\mathcal{D}_{1}$.

Finally, we have mentionned in Section 3 the existence of two $S l(2) \oplus S l(2)$ and two $S l(4) \equiv S O(6)$ algebras. The corresponding decompositions are:

$$
\begin{aligned}
& S l(2) \oplus S l(2)\left\{\begin{array}{l}
\underline{n}=2\left(\mathcal{D}_{1 / 2} \oplus \overline{\mathcal{D}}_{1 / 2}\right) \oplus(n-8) \mathcal{D}_{0} \\
\underline{n}=\mathcal{D}_{1} \oplus(n-3) \mathcal{D}_{0}
\end{array}\right. \\
& S l(4)\left\{\begin{array}{l}
\underline{n}=\mathcal{D}_{3 / 2} \oplus \overline{\mathcal{D}}_{3 / 2} \oplus(n-8) \mathcal{D}_{0} \\
\underline{n}=\mathcal{D}_{2} \oplus(n-5) \mathcal{D}_{0}
\end{array}\right.
\end{aligned}
$$

We remind that for each $S O(2 n)$ subalgebras, there exist $S l(2)$ algebras related to the singular embeddings $S O(2 k+1) \oplus S O(2 n-2 k-1), 0<2 k<n$.

\subsection{The $\mathcal{G}$ adjoint representation with respect to $S l(2)$ subalgebras}

To achieve the $S l(2)$ reduction of the adjoint representation for any simple Lie algebra $\mathcal{G}$ from the knowledge of the fundamental representation, the following 
formulae are especially convenient:

$$
\begin{aligned}
\left(\mathcal{D}_{n} \times \mathcal{D}_{n}\right)_{A} & =\mathcal{D}_{2 n-1} \oplus \mathcal{D}_{2 n-3} \oplus \cdots \oplus \mathcal{D}_{1} \quad n \in \mathbb{Z} \\
\left(\mathcal{D}_{n-(1 / 2)} \times \mathcal{D}_{n-(1 / 2)}\right)_{A} & =\mathcal{D}_{2 n-2} \oplus \mathcal{D}_{2 n-4} \oplus \cdots \oplus \mathcal{D}_{0} \quad n \in \mathbb{Z} \\
\left(\mathcal{D}_{n} \times \mathcal{D}_{n}\right)_{S} & =\mathcal{D}_{2 n} \oplus \mathcal{D}_{2 n-2} \oplus \cdots \oplus \mathcal{D}_{0} \quad n \in \mathbb{Z} \\
\left(\mathcal{D}_{n-(1 / 2)} \times \mathcal{D}_{n-(1 / 2)}\right)_{S} & =\mathcal{D}_{2 n-1} \oplus \mathcal{D}_{2 n-3} \oplus \cdots \oplus \mathcal{D}_{1} \quad n \in \mathbb{Z}
\end{aligned}
$$

the subscript (A) S standing for (Anti-)Symmetric part of the product. We have also, for $m, p \in \mathbb{Z}$ and $j, k \in \frac{1}{2} \mathbb{Z}$ :

$$
\begin{aligned}
\left\{\left(m \mathcal{D}_{j}\right) \times\left(m \mathcal{D}_{j}\right)\right\}_{A} & =\frac{m(m+1)}{2}\left(\mathcal{D}_{j} \times \mathcal{D}_{j}\right)_{A} \oplus \frac{m(m-1)}{2}\left(\mathcal{D}_{j} \times \mathcal{D}_{j}\right)_{S} \\
& =m\left(\mathcal{D}_{j} \times \mathcal{D}_{j}\right)_{A} \oplus \frac{m(m-1)}{2}\left(\mathcal{D}_{j} \times \mathcal{D}_{j}\right) \\
\left\{\left(m \mathcal{D}_{j}\right) \times\left(m \mathcal{D}_{j}\right)\right\}_{S} & =\frac{m(m+1)}{2}\left(\mathcal{D}_{j} \times \mathcal{D}_{j}\right)_{S} \oplus \frac{m(m-1)}{2}\left(\mathcal{D}_{j} \times \mathcal{D}_{j}\right)_{A} \\
& =m\left(\mathcal{D}_{j} \times \mathcal{D}_{j}\right)_{S} \oplus \frac{m(m-1)}{2}\left(\mathcal{D}_{j} \times \mathcal{D}_{j}\right) \\
\left\{\left(m \mathcal{D}_{j}\right) \times\left(p \mathcal{D}_{k}\right) \oplus\left(p \mathcal{D}_{k}\right) \times\left(m \mathcal{D}_{j}\right)\right\}_{A} & =\left\{\left(m \mathcal{D}_{j}\right) \times\left(p \mathcal{D}_{k}\right) \oplus\left(p \mathcal{D}_{k}\right) \times\left(m \mathcal{D}_{j}\right)\right\}_{S} \\
& =m p\left(\mathcal{D}_{j} \times \mathcal{D}_{k}\right)
\end{aligned}
$$

where $m \mathcal{D}_{j}$ stands for the direct sum of $m$ representations $\mathcal{D}_{j}$.

\subsection{Example: case of $S O(8)$}

We illustrate our methods on $S O(8)$. First, we give the decompositions of its fundamental and adjoint representations with respect to the $S l(2)$ subalgebras associated to regular subalgebras:

$$
\begin{aligned}
& S O(8) \quad \underline{8}=\mathcal{D}_{3} \oplus \mathcal{D}_{0} \quad(8 \times 8)_{A}=\mathcal{D}_{5} \oplus 2 \mathcal{D}_{3} \oplus \mathcal{D}_{1} \\
& S l(4) \quad \underline{8}=\mathcal{D}_{3 / 2} \oplus \overline{\mathcal{D}}_{3 / 2} \quad(8 \times 8)_{A}=\mathcal{D}_{3} \oplus 3 \mathcal{D}_{2} \oplus \mathcal{D}_{1} \oplus 3 \mathcal{D}_{0} \\
& S l(4)^{\prime} \quad \underline{8}=\mathcal{D}_{2} \oplus 3 \mathcal{D}_{0} \quad(8 \times 8)_{A}=\mathcal{D}_{3} \oplus 3 \mathcal{D}_{2} \oplus \mathcal{D}_{1} \oplus 3 \mathcal{D}_{0} \\
& \operatorname{Sl}(3) \quad \underline{8}=2 \mathcal{D}_{1} \oplus 2 \mathcal{D}_{0} \quad(8 \times 8)_{A}=\mathcal{D}_{2} \oplus 7 \mathcal{D}_{1} \oplus 2 \mathcal{D}_{0} \\
& S l(2) \quad \underline{8}=\mathcal{D}_{1 / 2} \oplus \overline{\mathcal{D}}_{1 / 2} \oplus 4 \mathcal{D}_{0} \quad(8 \times 8)_{A}=\mathcal{D}_{1} \oplus 8 \mathcal{D}_{1 / 2} \oplus 9 \mathcal{D}_{0} \\
& 4 S l(2) \quad \underline{8}=2 \mathcal{D}_{1} \oplus 2 \mathcal{D}_{0} \quad(8 \times 8)_{A}=\mathcal{D}_{2} \oplus 7 \mathcal{D}_{1} \oplus 2 \mathcal{D}_{0} \\
& 3 S l(2) \quad \underline{8}=\mathcal{D}_{1} \oplus 2 \mathcal{D}_{1 / 2} \oplus \mathcal{D}_{0} \quad(8 \times 8)_{A}=2 \mathcal{D}_{3 / 2} \oplus 3 \mathcal{D}_{1} \oplus 4 \mathcal{D}_{1 / 2} \oplus 3 \mathcal{D}_{0} \\
& 2 S l(2) \quad \underline{8}=\mathcal{D}_{1} \oplus 5 \mathcal{D}_{0} \quad(8 \times 8)_{A}=6 \mathcal{D}_{1} \oplus 10 \mathcal{D}_{0} \\
& (2 S l(2))^{\prime} \quad \underline{8}=2\left(\mathcal{D}_{1 / 2} \oplus \overline{\mathcal{D}}_{1 / 2}\right) \quad(8 \times 8)_{A}=6 \mathcal{D}_{1} \oplus 10 \mathcal{D}_{0}
\end{aligned}
$$

One remarks that the reductions associated to the two $S l(4)$ on the one hand, and the two $2 S l(2)$ on the other hand, give the same decomposition of the adjoint: this special case has to be related to the triality in $S O(8)$. 
Finally, one has to add the singular embedding:

$$
S O(3) \oplus S O(5) \quad \underline{8}=\mathcal{D}_{1} \oplus \mathcal{D}_{2} \quad(8 \times 8)_{A}=2 \mathcal{D}_{3} \oplus \mathcal{D}_{2} \oplus 3 \mathcal{D}_{1}
$$

\section{$5 \quad S l(2) \oplus U(1)_{Y}$ decompositions of simple Lie al- gebras}

\section{$5.1 S l(n)$ algebras}

We start by considering the case $\mathcal{G}=S l(n)$, which has already been studied in some detail in [8]. Let us recall that, for such an algebra, all the $S l(2)$ representations of equal dimension $\mathcal{D}_{j}$ have the same $U(1)_{Y}$ eigenvalue $y_{j}$ in the $\underline{n}$ fundamental representation, so that a general decomposition reads

$$
\underline{n}=\bigoplus_{j} n_{j} \mathcal{D}_{j}\left(y_{j}\right) \quad \text { with } \quad j^{\prime} s \quad \text { all different }
$$

where $n_{j}$ is the multiplicity of $\mathcal{D}_{j}$. One will have to impose to the product $\underline{n} \times \bar{n}-\mathcal{D}_{0}(0)$, the non degeneracy condition $|y| \leq j$ for any representation $\mathcal{D}_{j}(y)$ in the $\mathcal{G}$ adjoint representation. Note that the condition $y \in \frac{1}{2} \mathbb{Z}$, which ensures a (half-)integral gradation, has to be imposed only in the adjoint representation, and not in the fundamental.

As an example, consider the $S l(2)$ which is principal with respect to $A_{n}$ in $A_{n+2}$. Then

$$
\begin{aligned}
& \underline{n+3}=\mathcal{D}_{n / 2}(y) \oplus 2 \mathcal{D}_{0}\left(-\frac{n+1}{2} y\right) \\
& \overline{n+3}=\mathcal{D}_{n / 2}(-y) \oplus 2 \mathcal{D}_{0}\left(\frac{n+1}{2} y\right)
\end{aligned}
$$

where we have, of course, imposed the traceless condition for $Y$. It follows:

$\underline{n+3} \times \overline{n+3}-\mathcal{D}_{0}(0)=\left(\oplus_{j=1}^{n} \mathcal{D}_{j}(0)\right) \oplus 2 \mathcal{D}_{n / 2}\left(\frac{n+3}{2} y\right) \oplus 2 \mathcal{D}_{n / 2}\left(-\frac{n+3}{2} y\right) \oplus 4 \mathcal{D}_{0}(0)$

with the condition $\left|\frac{n+3}{2} y\right| \leq \frac{n}{2}$ and $y^{\prime}=\frac{n+3}{2} y \in \frac{1}{2} \mathbb{Z}$.

\section{$5.2 S O(n)$ algebras}

Now, let us turn to the $\mathcal{G}=B_{n}$ or $D_{n}$ case. These algebras have a real fundamental representation, so that if $\mathcal{D}_{j}(y), y \neq 0$, appears in the decomposition, then $\mathcal{D}_{j}(-y)$ must also be present with the same multiplicity. To get the adjoint 
representation, we have to improve the formulae (4.5-4.11) by specifying the $U(1)$ dependence. Using the reality of the adjoint representation, one is led to

$$
\begin{aligned}
& \left\{\left[n \mathcal{D}_{j}(y) \oplus n \mathcal{D}_{j}(-y)\right] \times\left[n \mathcal{D}_{j}(y) \oplus n \mathcal{D}_{j}(-y)\right]\right\}_{A}=n^{2}\left(\mathcal{D}_{j} \times \mathcal{D}_{j}\right)(0) \oplus \\
& \oplus\left(\left(n \mathcal{D}_{j}\right) \times\left(n \mathcal{D}_{j}\right)\right)_{A}(2 y) \oplus\left(\left(n \mathcal{D}_{j}\right) \times\left(n \mathcal{D}_{j}\right)\right)_{A}(-2 y) \quad \text { for } \quad j \in \frac{1}{2} \mathbb{Z} \quad \text { and } \quad n \in \mathbb{Z}
\end{aligned}
$$

where $\left(\left(n \mathcal{D}_{j}\right) \times\left(n \mathcal{D}_{j}\right)\right)_{A}$ is computed via (4.9). This formula shows that from a term $n \mathcal{D}_{j}(y)$ in the fundamental, we will always get a term $\mathcal{D}_{0}(2 y)$ in the adjoint, except if $n=1$ and $j$ is integer. Moreover, when $n=1$ and $j$ is integer but non zero, there will always exists a $\mathcal{D}_{1}( \pm 2 y)$ term in the adjoint representation. The non degeneracy condition $|y| \leq j$ for $\mathcal{D}_{j}(y)$ will then led to set $y=0$, except for $n=1$ and $j$ integer, where, for $j \neq 0$, we will have $|2 y| \leq 1$ and $2 y \in \frac{1}{2} \mathbb{Z}$, that is $y=0$, or $y= \pm \frac{1}{4}$, or $y= \pm \frac{1}{2}$.

Thus, for the orthogonal series, the only $S l(2)$ representation with non zero $U(1)$ eigenvalues are those which appear in the fundamental representation as $n\left(\mathcal{D}_{p}(y) \oplus \mathcal{D}_{p}(-y)\right)$ with $n=1$ and $p$ integer. Moreover, for $p \neq 0$, we have $|2 y|=0$, or $\frac{1}{2}$, or 1 .

Note that these restrictions are necessary but not sufficient conditions on $y$ : we still have to impose the non degeneracy condition in the $\mathcal{G}$ adjoint. To be complete, let us add the formula:

$$
\begin{aligned}
& \left\{2\left[n \mathcal{D}_{j}(y) \oplus n \mathcal{D}_{j}(-y)\right] \times\left[p \mathcal{D}_{k}\left(y^{\prime}\right) \oplus p \mathcal{D}_{k}\left(-y^{\prime}\right)\right]\right\}_{A}=\left(\mathcal{D}_{n} \times \mathcal{D}_{p}\right)\left(y+y^{\prime}\right) \oplus \\
& \oplus\left(\mathcal{D}_{n} \times \mathcal{D}_{p}\right)\left(-\left(y+y^{\prime}\right)\right) \oplus\left(\mathcal{D}_{n} \times \mathcal{D}_{p}\right)\left(y-y^{\prime}\right) \oplus\left(\mathcal{D}_{n} \times \mathcal{D}_{p}\right)\left(-\left(y-\left(\mathfrak{y}^{\prime} \cdot \grave{\phi}\right)\right)\right.
\end{aligned}
$$

As an example, we look at the principal $S l(2)$ of $S O(2 n-1)$ in $S O(2 n+1)$ :

$$
\begin{aligned}
& \underline{2 n+1}=\mathcal{D}_{n-1}(0) \oplus \mathcal{D}_{0}(y) \oplus \mathcal{D}_{0}(-y)=\overline{2 n+1} \\
& (\underline{2 n+1} \times \underline{2 n+1})_{A}=\left(\mathcal{D}_{2 n-3} \oplus \mathcal{D}_{2 n-1} \oplus \cdots \oplus \mathcal{D}_{1} \mathcal{D}_{0}\right)(0) \oplus \mathcal{D}_{n-1}(y) \oplus \mathcal{D}_{n-1}(-y)
\end{aligned}
$$

with the condition $|y| \leq n-1$.

\section{$5.3 \quad S p(2 n)$ algebras}

Finally, let us study the case $\mathcal{G}=C_{n}$. From the $S O(n)$ case, it is easy to deduce the rule:

$$
\begin{aligned}
& \left\{\left[n \mathcal{D}_{j}(y) \oplus n \mathcal{D}_{j}(-y)\right] \times\left[n \mathcal{D}_{j}(y) \oplus n \mathcal{D}_{j}(-y)\right]\right\}_{S}=n^{2}\left(\mathcal{D}_{j} \times \mathcal{D}_{j}\right)(0) \oplus \\
& \oplus\left(\left(n \mathcal{D}_{j}\right) \times\left(n \mathcal{D}_{j}\right)\right)_{S}(2 y) \oplus\left(\left(n \mathcal{D}_{j}\right) \times\left(n \mathcal{D}_{j}\right)\right)_{S}(-2 y) \quad \text { for } \quad j \in \frac{1}{2} \mathbb{Z} \quad \text { and } \quad n \in \mathbb{Z}
\end{aligned}
$$


where $\left(\left(n \mathcal{D}_{j}\right) \times\left(n \mathcal{D}_{j}\right)\right)_{A}$ is computed via $(4.10)$.

Then, the $S l(2) \oplus U(1)$ decomposition of $C_{n}$ is deduced from the $B_{n}$ one by exchanging integer and half-integer:

For the symplectic series, the only $S l(2)$ representations with non zero $U(1)$ eigenvalues are those which appear in the fundamental representation as $n\left(\mathcal{D}_{p+\frac{1}{2}}(y) \oplus\right.$ $\left.\mathcal{D}_{p+\frac{1}{2}}(-y)\right)$ with $n=1$ and $p$ integer. Moreover, the allowed eigenvalues for the $U(1)$ generator $Y$ are $|2 y|=0$, or $\frac{1}{2}$, or 1 .

We illustrate these results on the decomposition under the $S l(2)$ of $S l(2)^{2} \oplus$ $S p(2 n-2)$ in $S p(2 n+2)$ :

$$
\begin{aligned}
\frac{2 n+2}{\overline{2 n+2}}= & \mathcal{D}_{n-(3 / 2)}(0) \oplus \mathcal{D}_{\frac{1}{2}}(y) \oplus \mathcal{D}_{\frac{1}{2}}(-y) \\
(\underline{2 n+2} \times \overline{2 n+2})_{S}= & \left(\mathcal{D}_{2 n-3} \oplus \mathcal{D}_{2 n-5} \oplus \cdots \oplus \mathcal{D}_{1}\right)(0) \oplus\left(\mathcal{D}_{1} \oplus \mathcal{D}_{0}\right)(0) \oplus \\
& \oplus \mathcal{D}_{1}(2 y) \oplus \mathcal{D}_{1}(-2 y) \oplus\left(\mathcal{D}_{n-1} \oplus \mathcal{D}_{n-2}\right)(y) \oplus\left(\mathcal{D}_{n-1} \oplus \mathcal{D}_{n-2}\right)(-y)
\end{aligned}
$$

with $|2 y| \leq 1$.

\subsection{The algebra $G_{2}$}

This (rank 2) algebra admits the system of roots:

$$
\pm\left(e_{i} \pm e_{j}\right) \quad ; \quad \pm\left(2 e_{i}-e_{j}-e_{k}\right) \quad \text { with } \quad i, j, k=1,2,3 \quad \text { all different }
$$

The fundamental representation of $G_{2}$ is seven-dimensional, and its adjoint has the dimension 14. These representations are real. To simplify the discussion about $S l(2) \oplus U(1)$ decomposition, we remark that $G_{2}$ can be embedded in $S O(7)$ (in a singular way). As a consequence, its adjoint representation will be present in the antisymmetric part of the product $\underline{7} \times \underline{7}$. Indeed, we have [17:

$$
(\underline{7} \times \underline{7})_{A}=\underline{7} \oplus \underline{14}
$$

Thus, we can obtain the adjoint representation from the fundamental by $\underline{14}=(\underline{7} \times$ 7) ${ }_{A}-\underline{7}$. It is then sufficient to know the decomposition of the fundamental. This is done with the same rules as for the $S O(n)$ algebras (because of the embedding $\left.G_{2} \subset S O(7)\right)$. Note that none of the $S l(2)$ subalgebras of $G_{2}$ can be extended to a $S l(2) \oplus U(1)$ subalgebra in such a way that (2.19) is still satisfied. The results are presented in table 8 . The defining vector is given in the Cartan basis of $S O(7)$, the Cartan generators of $G_{2}$ being given by $H_{1}-H_{2}$ and $2 H_{2}-H_{1}-H_{3}$ (see section 6). 


\subsection{The algebra $F_{4}$}

This exceptional algebra has rank 4 and dimension 52. Its fundamental representation has dimension 26 , and $F_{4}$ can be (irregularily) embedded in $S O(26)$. However, one cannot directly obtains the adjoint representation from the fundamental one, since a new representation appears in the antisymmetric part of the product:

$$
(26 \times 26)_{A}=52+273
$$

Thus, the only information on the $U(1)$ dependence comes from the non degeneracy condition (2.19). The $S l(2)$ algebras has already been studied in [11, where the decomposition of the fundamental representation was given: we recall in table 9 this decomposition giving the conformal spin content.

\section{Classification of (half-)integral gradings.}

The decomposition of the adjoint of a simple Lie algebra $\mathcal{G}$ in terms of $S l(2) \oplus$ $U(1)$ representations gives an exhaustive classification of the different constrained WZW theory arising from a (half-)integral grading. Moreover, the different values of $Y$ (at fixed $S l(2)$ subalgebra) leads to the equivalent theories [7]. Thus, if we know how to reconstruct the gradation $H$ from this decomposition, we will be able to give an explicit classification of gradations. This is the aim of this section.

\subsection{Defining vectors}

An $S l(2)$ algebra in a simple Lie algebra $\mathcal{G}$ is specified [11] by its defining vector $\left(f_{1}, \ldots, f_{r}\right)$, itself defined from the relation

$$
M_{0}=\sum_{i=1}^{r} f_{i} H_{i} \quad f_{i} \quad \text { rational }
$$

where $M_{0}$ denotes the Cartan part of $S l(2)$ and $\left\{H_{1}, \ldots, H_{r}\right\}$ a Cartan subalgebra of $\mathcal{G}$. For the A,B,C,D algebras of rank up to 6 , a defining vector for all $S l(2)$ subalgebras has been explicitely computed in [12], and we will use the same normalization here, up to a global factor 2. Let us precise the Cartan basis for these algebras.

For $B_{n}\left(D_{n}\right)$ algebras, where the roots are $\pm e_{i} \pm e_{j}$ and $\pm e_{i}\left( \pm e_{i} \pm e_{j}\right) i, j=$ $1, \ldots, n$. A basis of simple roots is given by $e_{i}-e_{i+1}(i=1, \ldots, n-1)$ and $e_{n}$ $\left(e_{n-1}+e_{n}\right)$ for $B_{n}\left(D_{n}\right)$. The Cartan generators satisfy

$$
\left[H_{i}, E_{e_{j}}\right]=\delta_{i j} E_{e_{j}} \forall i, j=1, \ldots, n
$$


For $C_{n}$ algebras, the roots are $\pm e_{i} \pm e_{j}$ and $\pm 2 e_{i} i, j=1, \ldots, n$, a basis of simple roots being $e_{i}-e_{i+1} i=1, . . n-1$ and $2 e_{n}$. The Cartan generators satisfy

$$
\left[H_{i}, E_{2 e_{j}}\right]=2 \delta_{i j} E_{2 e_{j}} \quad \forall i, j=1, \ldots, n
$$

For $A_{n}$ algebras, the $H_{i}$ generators (with $i=1, \ldots, n+1$ ) satisfy

$$
\left[H_{i}, E_{e_{j}-e_{k}}\right]=\left(\delta_{i j}-\delta_{i k}\right) E_{e_{j}-e_{k}} \forall i, j, k=1, \ldots, n+1 \quad \text { with } \quad j \neq k
$$

where the roots are $\pm\left(e_{i}-e_{j}\right) i, j=1, \ldots, n+1$ and the Cartan generators take the form $H_{i}-H_{j}, i \neq j$. Note that here, the definition of the defining vector is slightly modified, since it has a dimension which is no longer the rank of $A_{n}$, but the rank plus one. The tracelessness condition is then used to recover the rank.

The defining vectors can always be chosen such that

$$
f_{n} \leq f_{n-1} \leq \cdots \leq f_{1} \quad \text { if } \quad f=\left(f_{1}, f_{2}, \ldots, f_{n}\right)
$$

Moreover, for $\mathcal{G}=B_{n}, C_{n}, D_{n}$, we have

$$
f_{i} \geq 0 \quad \forall i
$$

For $\mathcal{G}=A_{n},(6.6)$ becomes the tracelessness condition

$$
\sum_{i=1}^{n+1} f_{i}=0
$$

When adopting this convention for the defining vector, the gradation is such that the simple roots introduced above have a positive grade.

If $f^{\prime}=\left(f_{1}^{\prime}, f_{2}^{\prime}, \ldots, f_{p}^{\prime}, 0, . ., 0\right)$ and $f^{\prime \prime}=\left(f_{1}^{\prime \prime}, f_{2}^{\prime \prime}, \ldots, f_{q}^{\prime \prime}, 0, . ., 0\right)$ are the defining vectors associated to the two principal $S l(2)$ 's of two subalgebras $\mathcal{G}^{\prime}$ and $\mathcal{G}^{\prime \prime}$ of $\mathcal{G}$, and if $\mathcal{G}^{\prime} \oplus \mathcal{G}^{\prime \prime}$ is also a subalgebra of $\mathcal{G}$, then a defining vector of the principal $S l(2)$ of $\mathcal{G}^{\prime} \oplus \mathcal{G}^{\prime \prime}$ is $f=\left(f_{1}, \ldots, f_{p+q}\right)$, where the set $\left\{f_{1}, \ldots, f_{p+q}\right\}$ is the union of the sets $\left\{f_{1}^{\prime}, \ldots, f_{p}^{\prime}\right\}$ and $\left\{f_{1}^{\prime \prime}, \ldots, f_{q}^{\prime \prime}\right\}$, and can be arranged according to (6.5).

\subsection{Calculation of the defining vector from the $S l(2)$ de- composition}

We set for a while $Y=0$, and look at the gradation produced by $M_{0}$, Cartan generator of a given $S l(2)$ subalgebra of $\mathcal{G}$. This $S l(2)$ subalgebra can always be seen as the principal embedding of a (regular or singular) subalgebra of $\mathcal{G}$. From the properties mentionned above, we just have to specify the defining vector associated to simple subalgebras (except the case $2 A_{1}$ in $S O(n)$, see below). Due 
to the Cartan basis we have adopted, the defining vectors are directly related to the eigenvalues of $M_{0}$ in the fundamental representation of $\mathcal{G}$.

First consider the case $\mathcal{G}=A_{n}$. The defining vector components are just the eigenvalues of $M_{0}$, since one can always diagonalize $M_{0}$ with hermitian matrices. Then, we have the rules:

$$
\begin{aligned}
A_{2 p} \subset A_{n} & \longrightarrow f=(p, p-1, \ldots, 1, \underbrace{0, \ldots, 0}_{n+1-2 p},-1,-2, \ldots,-p) \\
A_{2 p+1} \subset A_{n} & \longrightarrow f=(p+\frac{1}{2}, p-\frac{1}{2}, \ldots, \frac{1}{2}, \underbrace{0, \ldots, 0}_{n-2 p-1}, \frac{-1}{2}, \frac{-3}{2}, \ldots,-p-\frac{1}{2})
\end{aligned}
$$

For example, the defining vector of $A_{2}$ (resp. $\left.A_{1}\right)$ in $A_{4}$ is $(1,0,0,0,-1)$ (resp. $\left.\left(\frac{1}{2}, 0,0,0, \frac{-1}{2}\right)\right)$. The defining vector of $A_{2} \oplus A_{1}$ is $\left(1, \frac{1}{2}, 0,-\frac{1}{2},-1\right)$.

Let us now turn to the $S O(n)$ case. Because of the antisymmetry of the matrices in the fundamental representation, the Cartan generators cannot be diagonal. In fact, they are constructed with $\sigma_{2}$ matrices on the diagonal. Each $\sigma_{2}$ matrix possesses +1 and -1 as eigenvalues, so that one has only to specify the positive $M_{0}$-eigenvalues in the defining vector. The general rules are:

$$
\begin{aligned}
B_{p} \quad \text { or } \quad D_{p+1} \subset B_{n} & \longrightarrow f=(p, p-1, \ldots, 1,0, \ldots 0) \\
D_{p+1} & \subset D_{n} \longrightarrow f=(p, p-1, \ldots, 1,0, \ldots 0) \\
A_{2 p} \subset B_{n} \quad \text { or } \quad D_{n} & \longrightarrow f=(p, p, p-1, p-1, \ldots, 1,1,0, \ldots 0) \\
A_{2 p+1} \subset B_{n} \quad \text { or } \quad D_{n} \quad \longrightarrow f & =\left(p+\frac{1}{2}, p+\frac{1}{2}, p-\frac{1}{2}, p-\frac{1}{2}, \ldots, \frac{1}{2}, \frac{1}{2}, 0,\right.
\end{aligned}
$$

As there are some exceptional embeddings of $S l(2)$ algebras in orthogonal ones, there will be also exceptions for the defining vectors. For $A_{3} \equiv D_{3}$, they are two different defining vectors, one associated to " $A_{3}$ ", and the other one to " $D_{3}$ ":

$$
\begin{array}{ll}
" A_{3} " \subset B_{n} & \text { or } \quad D_{n} \longrightarrow f=\left(\frac{3}{2}, \frac{3}{2}, \frac{1}{2}, \frac{1}{2}, 0, \ldots 0\right) \\
" D_{3} " \subset B_{n} & \text { or } \quad D_{n} \longrightarrow f=(2,1,0, \ldots 0)
\end{array}
$$

They are also two defining vectors for $2 A_{1} \subset S O(m)$

$$
\begin{array}{ll}
" 2 A_{1} " \subset B_{n} & \text { or } \quad D_{n} \quad \longrightarrow\left(\frac{1}{2}, \frac{1}{2}, \frac{1}{2}, \frac{1}{2}, 0, \ldots, 0\right) \\
" 2 A_{1} " \subset B_{n} & \text { or } \quad D_{n} \quad \longrightarrow(1,0, \ldots, 0)
\end{array}
$$

Finally, for the short root of $B_{n}$, we have

$$
A_{1}^{2} \subset B_{n} \longrightarrow(1,0, \ldots, 0)
$$


The defining vectors associated to the singular embeddings $\left(B_{i} \oplus B_{j}\right) \subset D_{n}$ (with $i+j=n-1, i \neq j)$ are computed with the above rules.

Finally, we study the case of $S p(2 n)$ algebras. The rules are similar to those of $S O(n)$ algebras:

$$
\begin{aligned}
& A_{1}^{1} \subset C_{n} \longrightarrow f=\left(\frac{1}{2}, 0, \ldots, 0\right) \\
& A_{2 p}^{2} \subset C_{n} \longrightarrow f=(p, p, p-1, p-1, \ldots, 1,1,0, \ldots 0) \\
& A_{2 p+1}^{2} \subset C_{n} \longrightarrow f=\left(p+\frac{1}{2}, p+\frac{1}{2}, p-\frac{1}{2}, p-\frac{1}{2}, \ldots, \frac{1}{2}, \frac{1}{2}, 0, \ldots 0\right) \\
& C_{p} \subset C_{n} \longrightarrow f=\left(p+\frac{1}{2}, p-\frac{1}{2}, \ldots, \frac{1}{2}, 0, \ldots 0\right)
\end{aligned}
$$

\subsection{Case of $S l(2) \oplus U(1)$ decomposition}

When $H \neq M_{0}$, we can no longer speak about defining vector for $H$, since $H$ cannot be embedded in an $S l(2)$ algebra. However, it is still possible to compute a vector $f=\left(f_{1}, \ldots, f_{n}\right)$ that defines $H$, using the relation (6.1). We give hereafter the rules to compute this vector associated to $H$.

Let us first look at the $S O(n)$ case, where $Y$ appears, in the fundamental representation, only in combinations $\mathcal{D}_{m}(y) \oplus \mathcal{D}_{m}(-y)$ with $m$ integer. The rule is then

$$
\begin{aligned}
& \mathcal{D}_{m}(y) \oplus \mathcal{D}_{m}(-y) \text { in } \text { Fund }^{\mathrm{l}}\left(m \in \mathbb{Z}_{+}\right) \\
& \longrightarrow f=(m+y, m-y, m-1+y, m-1-y, \ldots, 1+y, 1-y, y, 0, \ldots, 0)
\end{aligned}
$$

before ordering following (6.5). For example, for $A_{4} \subset D_{6}$, we have

$$
\begin{aligned}
& \underline{12}=\mathcal{D}_{2}\left(y_{2}\right) \oplus \overline{\mathcal{D}}_{2}\left(-y_{2}\right) \oplus \mathcal{D}_{0}\left(y_{0}\right) \oplus \overline{\mathcal{D}}_{0}\left(-y_{0}\right) \\
& f=\left(2+y_{2}, 2-y_{2}, 1+y_{2}, 1-y_{2}, y_{2}, y_{0}\right)
\end{aligned}
$$

For the case $\mathcal{G}=A_{n}$, the defining vector can be read directly in the fundamental decomposition: the piece corresponding to a representation $\mathcal{D}_{i}\left(y_{i}\right)$ in the fundamental is $\left(i+y_{i}, i-1+y_{i}, \ldots,-i+y_{i}\right)$. Note that the different eigenvalues $y_{i}$ are related by a traceless condition:

$$
\sum_{i} m_{i}(2 i+1) y_{i}=0 \quad \text { for } \quad \underline{n+1}=\bigoplus_{i} m_{i} \mathcal{D}_{i}\left(y_{i}\right)
$$

They are determined in the adjoint representation, by the usual condition $|y| \leq j$ and $y \in \frac{1}{2} \mathbb{Z}$ for any representation $\mathcal{D}_{j}(y)$ in the adjoint. 
For example, for the reduction of $A_{2}$ with respect to its regular $A_{1}$ algebra, we have

$$
\begin{aligned}
& \underline{3}=\mathcal{D}_{\frac{1}{2}}(y) \oplus \mathcal{D}_{0}(-2 y) \quad \text { thus } \quad f=\left(\frac{1}{2}+y,-\frac{1}{2}+y,-2 y\right) \\
& \underline{8}=\left(\mathcal{D}_{1} \oplus \mathcal{D}_{0}\right)(0) \oplus \mathcal{D}_{\frac{1}{2}}(3 y) \oplus \mathcal{D}_{\frac{1}{2}}(-3 y) \\
& | \pm 3 y| \leq \frac{1}{2} \quad \text { and } \quad \pm 3 y \in \frac{1}{2} \mathbb{Z} \Rightarrow y=0, \pm \frac{1}{6}
\end{aligned}
$$

Finally, for the symplectic algebras, the rules are analogous to those of the $B_{n}$ case, that is:

$$
\begin{aligned}
& \mathcal{D}_{m+\frac{1}{2}}(y) \oplus \mathcal{D}_{m+\frac{1}{2}}(-y) \text { in Fund }{ }^{\mathrm{l}}\left(m \in \mathbb{Z}_{+}\right) \\
& \longrightarrow f=\left(m+\frac{1}{2}+y, m+\frac{1}{2}-y, m-\frac{1}{2}+y, m-\frac{1}{2}-y, \ldots, \frac{1}{2}+y, \frac{1}{2}-y, 0, \ldots, 0\right)
\end{aligned}
$$

\section{Poisson brackets of $W$ algebras}

\subsection{Generalities}

The knowledge of the spin contents of a $W$ algebra with the use of a $\operatorname{Sl}(2) \oplus$ $U_{Y}(1)$ decomposition, together with the Proposition 4 of section 2.2, allows us to determine many of the PB of this algebra, when $Y$ exists. Indeed, let $W_{I}$ be the $W$ generators, $I \in \mathcal{I}$ indexing the generators. The theory possesses a grading operator $H$, and we suppose here that $H \neq M_{0}$. The spin content associated to the stress energy tensor $T_{H}$ is then given by $s_{I}=1+j_{I}+y_{I}$. It is conserved through the $\mathrm{PB}$, so that starting from the general form:

$$
\begin{aligned}
& \left\{W_{I}(x), W_{J}\left(x^{\prime}\right)\right\}_{P B}=\sigma_{0}(I, J) \partial^{j_{I}+y_{I}+j_{J}+y_{J}+1} \delta\left(x-x^{\prime}\right)+ \\
& \quad+\sum_{K} \sum_{p, q} \sigma_{1}(I, J, K, p, q)\left(\partial^{p} W_{K}\left(x^{\prime}\right)\right)\left(\partial^{q} \delta\left(x-x^{\prime}\right)\right)+ \\
& \quad+\sum_{K, L} \sum_{p, q, r} \sigma_{2}(I, J, K, L, p, q, r)\left(\partial^{p} W_{K}\left(x^{\prime}\right)\right)\left(\partial^{r} W_{L}\left(x^{\prime}\right)\right)\left(\partial^{r} \delta\left(x-x^{\prime}\right)\right)+ \\
& \quad \vdots
\end{aligned}
$$

where the $\sigma_{n}(.$.$) are coefficients, the conformal invariance imposes the sums to$ satisfy the equalities

$$
\begin{aligned}
& p, q, K \quad \text { such that } \quad p+j_{K}+y_{K}+q=j_{I}+y_{I}+j_{J}+y_{J} \\
& p, q, r, K, L \quad \text { such that } \quad p+j_{K}+y_{K}+q+j_{L}+y_{L}+r+1=j_{I}+y_{I}+j_{J}+y_{J} \\
& \vdots
\end{aligned}
$$


But Proposition 4 ensures that this algebra is the same as the one obtained from the grading operator $M_{0}$. The main change between these two algebras is the stress energy tensor $\left(T_{H}\right.$ or $\left.T_{M_{0}}\right)$. Then, the conformal invariance of the $\mathrm{PB}$ when the gradation is given by $M_{0}$ imposes:

$$
\begin{aligned}
& p+j_{K}+q=j_{I}+j_{J} \\
& p+j_{K}+q+j_{L}+r+1=j_{I}+j_{J}
\end{aligned}
$$

Gathering (7.1) and (7.2) leads to:

$$
\begin{aligned}
& p+j_{K}+q=j_{I}+j_{J} \quad \text { and } \quad y_{K}=y_{I}+y_{J} \\
& p+j_{K}+q+j_{L}+r+1=j_{I}+j_{J} \quad \text { and } \quad y_{K}+y_{L}=y_{I}+y_{J}
\end{aligned}
$$

For each line, the second equality shows that the charge associated to the $U(1)_{Y}$ generator is conserved. This severely limits the number of allowed fields in the r.h.s. of the $\mathrm{PB}$, since not only the $T_{M_{0}}$-conformal spin (associated to $S l(2)$ ) but also the "hypercharge" associated to $Y$ is conserved. Note that in this context, $T_{M_{0}}$ has a zero $U(1)_{Y}$ value.

Finally, let us add that there may exist several independent Cartan generators $Y_{i}$ which can be added to $M_{0}$ in such a way that $H_{i}=M_{0}+Y_{i}$ is a non degenerate gradation, the corresponding $S l(2)$ subalgebra of which is still $\left(M_{ \pm}, M_{0}\right)$. For example, in the decomposition of $S O(8)$ with respect to $S l(3)$ (see section 4.3), we have

$$
\begin{aligned}
& \underline{8}=\mathcal{D}_{1}\left(y_{1}\right) \oplus \mathcal{D}_{1}\left(-y_{1}\right) \oplus \mathcal{D}_{0}\left(y_{0}\right) \oplus \mathcal{D}_{0}\left(-y_{0}\right) \\
& (8 \times 8)_{A}=\left(\mathcal{D}_{2} \oplus \mathcal{D}_{1} \oplus 2 \mathcal{D}_{0}\right)(0) \oplus \mathcal{D}_{1}\left(2 y_{1}\right) \oplus \mathcal{D}_{1}\left(-2 y_{1}\right) \oplus \\
& \oplus \mathcal{D}_{1}\left(y_{1}+y_{0}\right) \oplus \mathcal{D}_{1}\left(-\left(y_{1}+y_{0}\right)\right) \oplus \mathcal{D}_{1}\left(y_{1}-y_{0}\right) \oplus \mathcal{D}_{1}\left(-\left(y_{1}-y_{0}\right)(7.3)\right.
\end{aligned}
$$

In the above decomposition of the adjoint representation, one sees that $y_{0}$ and $y_{1}$ can take the values $0, \frac{1}{2}$, independently from one another, without violating the non degeneracy condition. So, we can decompose $Y$ in $Y_{0}+Y_{1}, Y_{0}$ and $Y_{1}$ being defined by the vectors $f_{0}=\left(\frac{3}{2}, \frac{1}{2}, \frac{1}{2}, 0\right)$ and $f_{1}=\left(1,1, \frac{1}{2}, 0\right)$.

Thus, we will now write the $W$ generators as

$$
W_{j+y+1} \equiv W_{j+1}^{(\vec{y})}
$$

$j+1$ being the conformal spin in the basis where all the fields (but $T$ ) are primary, and $\vec{y}$ being the set of "hypercharges" associated to the different possible $U(1)_{Y}$.

\footnotetext{
${ }^{3}$ This can be guessed if one remarks that the $S l(2)$ highest weights are the same for $H=M_{0}$ and $H=M_{0}+Y$.
} 
For instance, in the case of $S O(8)$ reduced by $S l(3)$, we will have as $W$ generators:

$$
\begin{array}{llll}
T_{M_{0}}^{(0,0)}, & W_{1}^{Y 1}, W_{1}^{Y 2} \\
W_{3}^{(0,0)}, & W_{2}^{(1,0)}, W_{2}^{(-1,0)} \\
W_{2}^{(1 / 2,1 / 2)} & , \quad W_{2}^{(-1 / 2,-1 / 2)} \quad, \quad W_{2}^{(1 / 2,-1 / 2)}, W_{2}^{(-1 / 2,1 / 2)}
\end{array}
$$

where the doublet superscript indicates the hypercharges of the $W$ generator with respect to $W_{1}^{Y 1}$ and $W_{1}^{Y 2}$.

\subsection{Use of the stress-energy tensor}

We know that the theory associated to $H$ contains a stress-energy tensor $T_{H}$, and that all the fields but $W_{1}^{Y}$ are primary. Moreover, from the equation (2.6), it is clear that

$$
T_{H}=T_{M_{0}}+\partial W_{1}^{Y} \quad \text { for } \quad H=M_{0}+Y
$$

Then, a generator $W_{j}^{(y)}$ being primary (we omit $T$ and $W_{1}^{Y}$ ) with respect to $T_{H}$ and $T_{M_{0}}$, we will have

$$
\left\{\partial_{x} W_{1}^{Y}(x), W_{j}^{(y)}\left(x^{\prime}\right)\right\}_{P B}=y W_{j}^{(y)}\left(x^{\prime}\right) \partial_{x} \delta\left(x-x^{\prime}\right)
$$

Note that although $T_{M_{0}}$ is not an eigenvector of $W_{Y}^{1}$, we associated to it an "eigenvalue" 0 . Of course, if there are several $U(1)$, each of them will satisfy this property.

Thus, the generator $W_{1}^{Y}$ associated to $Y=H-M_{0}$ generates a conserved "hypercharge", and all the $W$ generators except $T$ are $W_{1}^{Y}$-eigenvectors:

$$
\left\{W_{1}^{Y}(x), W_{j}^{(y)}\left(x^{\prime}\right)\right\}_{P B}=y W_{j}^{(y)}\left(x^{\prime}\right) \delta\left(x-x^{\prime}\right)
$$

$T$ possesses a zero hypercharge, but the PB reads:

$$
\left\{T(x), W_{1}^{Y}\left(x^{\prime}\right)\right\}_{P B}=\partial W_{1}^{Y}\left(x^{\prime}\right) \delta\left(x-x^{\prime}\right)+W_{1}^{Y}\left(x^{\prime}\right) \partial \delta\left(x-x^{\prime}\right)
$$

Finally, let us remark that the set of spin 1 generators must be closed, because of the conservation of the conformal spin. This shows that we will have a KM algebra, corresponding to the part of $\mathcal{G}$ which has not been used for the definition of the $S l(2)$ algebra. 


\subsection{Example}

As an example, let us look at the $W$ algebra coming from non Abelian Toda on $\operatorname{Sl}(3)$. The $W$ generators are

$$
W_{2}, W_{3 / 2+y}, W_{3 / 2-y}, W_{1} \quad \text { with } y=0 \quad \text { or } \quad \frac{1}{2}
$$

Applying the above procedure to the PB of this algebra, we can determine their structure. As a notation, we will write $\partial$ for $\partial_{x}$ and $\partial^{\prime}$ for $\partial_{x^{\prime}}$.

$$
\begin{aligned}
\left\{W_{2}(x), W_{2}\left(x^{\prime}\right)\right\}_{P B}= & \left(a_{1} \partial^{\prime} W_{2}\left(x^{\prime}\right)+a_{3} \partial^{\prime 2} W_{1}\left(x^{\prime}\right)+a_{4} \partial^{\prime}\left(W_{1} W_{1}\right)\left(x^{\prime}\right)\right. \\
& \left.+a_{2} W_{3 / 2+y} W_{3 / 2-y}\left(x^{\prime}\right)\right) \delta\left(x-x^{\prime}\right) \\
& +\left(a_{5} W_{2}\left(x^{\prime}\right)+a_{6} W_{1} W_{1}\left(x^{\prime}\right)+a_{7} W_{1}\left(x^{\prime}\right)\right) \partial \delta\left(x-x^{\prime}\right) \\
& +a_{8} W_{1}\left(x^{\prime}\right) \partial^{2} \delta\left(x-x^{\prime}\right)+a_{9} \partial^{3} \delta\left(x-x^{\prime}\right) \\
\left\{W_{2}(x), W_{3 / 2 \pm y}\left(x^{\prime}\right)\right\}_{P B}= & a_{10} \partial^{\prime} W_{3 / 2 \pm y}\left(x^{\prime}\right) \delta\left(x-x^{\prime}\right)+a_{11} W_{3 / 2 \pm y}\left(x^{\prime}\right) \partial \delta\left(x-\left(x^{\prime} .11\right)\right. \\
\left\{W_{2}(x), W_{1}\left(x^{\prime}\right)\right\}_{P B}= & \left(a_{12} \partial^{\prime} W_{1}\left(x^{\prime}\right)+a_{13} \partial^{\prime} W_{2}\left(x^{\prime}\right)\right) \delta\left(x-x^{\prime}\right)+ \\
& +a_{14} W_{1}\left(x^{\prime}\right) \partial \delta\left(x-x^{\prime}\right)+a_{15} \partial^{2} \delta\left(x-x^{\prime}\right) \\
\left\{W_{3 / 2 \pm y}(x), W_{3 / 2 \pm y}\left(x^{\prime}\right)\right\}_{P B}= & 0 \\
\left\{W_{3 / 2+y}(x), W_{3 / 2-y}\left(x^{\prime}\right)\right\}_{P B}= & \left(a_{16} \partial^{\prime} W_{1}\left(x^{\prime}\right)+a_{17} W_{1} W_{1}\left(x^{\prime}\right)+a_{18} W_{2}\left(x^{\prime}\right)\right) \delta\left(x-x^{\prime}\right)+ \\
& +a_{19} W_{1}\left(x^{\prime}\right) \partial \delta\left(x-x^{\prime}\right)+a_{20} \partial^{2} \delta\left(x-x^{\prime}\right) \\
\left\{W_{1}(x), W_{3 / 2 \pm y}\left(x^{\prime}\right)\right\}_{P B}= & a_{21}^{ \pm} W_{3 / 2 \pm y}\left(x^{\prime}\right) \delta\left(x-x^{\prime}\right) \\
\left\{W_{1}(x), W_{1}\left(x^{\prime}\right)\right\}_{P B}= & a_{22} \partial \delta\left(x-x^{\prime}\right)
\end{aligned}
$$

Now, assuming that $Y=0$, replacing $W_{2}$ by $T$ the Virasoro tensor, and recognizing in $W_{1}$ the $W_{1}^{Y}$ generator, we are led to the constraints:

$$
\begin{aligned}
& a_{1}=1, a_{5}=2, a_{2}=a_{3}=a_{4}=a_{6}=a_{7}=a_{8}=0 \\
& a_{10}=1, a_{11}=\frac{3}{2} \\
& a_{12}=1, a_{14}=1, a_{13}=a_{15}=0 \\
& a_{21}^{ \pm}= \pm 1
\end{aligned}
$$

Thus, the $W$ algebra associated to the regular $S l(2)$ in $S l(3)$ must satisfy:

$$
\begin{array}{rlr}
\left\{T(x), T\left(x^{\prime}\right)\right\}_{P B}= & \partial^{\prime} T\left(x^{\prime}\right) \delta\left(x-x^{\prime}\right)+2 T\left(x^{\prime}\right) \partial \delta\left(x-x^{\prime}\right)+c \partial^{3} \delta\left(x-\left(x^{2}\right) 1\right) \\
\left\{T(x), W_{3 / 2}^{ \pm}\left(x^{\prime}\right)\right\}_{P B}= & \partial^{\prime} W_{3 / 2}^{ \pm}\left(x^{\prime}\right) \delta\left(x-x^{\prime}\right)+\frac{3}{2} W_{3 / 2}^{ \pm}\left(x^{\prime}\right) \partial \delta\left(x-x^{\prime}\right) & (7.22) \\
\left\{T(x), W_{1}\left(x^{\prime}\right)\right\}_{P B}= & \partial^{\prime} W_{1}\left(x^{\prime}\right) \delta\left(x-x^{\prime}\right)+W_{1}\left(x^{\prime}\right) \partial \delta\left(x-x^{\prime}\right) & (7.23) \\
\left\{W_{3 / 2}^{ \pm}(x), W_{3 / 2}^{ \pm}\left(x^{\prime}\right)\right\}_{P B}= & 0 & (7.24) \\
\left\{W_{3 / 2}^{+}(x), W_{3 / 2}^{-}\left(x^{\prime}\right)\right\}_{P B}= & \left(a_{16} \partial^{\prime} W_{1}\left(x^{\prime}\right)+a_{17} W_{1} W_{1}\left(x^{\prime}\right)+a_{18} T\left(x^{\prime}\right)\right) \delta\left(x-x^{\prime}\right)+ \\
& +a_{19} W_{1}\left(x^{\prime}\right) \partial \delta\left(x-x^{\prime}\right)+a_{20} \partial^{2} \delta\left(x-x^{\prime}\right) & (7.25) \\
\left\{W_{1}(x), W_{3 / 2}^{ \pm}\left(x^{\prime}\right)\right\}_{P B}= & \pm W_{3 / 2}^{ \pm}\left(x^{\prime}\right) \delta\left(x-x^{\prime}\right) & \\
\left\{W_{1}(x), W_{1}\left(x^{\prime}\right)\right\}_{P B}= & k \partial \delta\left(x-x^{\prime}\right) &
\end{array}
$$


which has to be compared with the $W$ algebra explicited in [15]. Note that the Jacobi identities for the $\mathrm{PB}$ of the $W$ algebra will also constrain the remaining structure constants.

\section{$8 W$ algebras from Lie algebras of rank up to 4}

As an application of the above formulation, we present here an exhaustive classification of $W$ algebras arising from constrained WZW models based on classical algebras of rank up to 4 . For such a purpose, we follow the point of view developped in section 2.4, using the results presented in sections 3-6. Although the algebras $B_{2}$ and $C_{2}$ on the one hand, and $A_{3}$ and $D_{3}$ on the other hand are isomorphic, we have separately considered these four algebras to show the differences in the calculations. The classification is listed in tables 119, where the decomposition of the fundamental of $\mathcal{G}$ with respect to $S l(2) \oplus U(1)$ is given. We give the minimal (i.e. the lowest dimensional) regular subalgebras containing the $S l(2)$, when they exist. For the singular embedding associated to $D_{4}$, we mention the $S O(3) \oplus S O(5)$ subalgebra. Then, we give the conformal spin content $s=j+1$, with the convention: $n * s$ means that the spin $s$ appears $n$ times. In the same column, we give under the spin $s$ the hypercharge(s) $y$ when it exists. Finally, we write the different gradations that lead to this $W$ algebra. 


\begin{tabular}{|c|c|c|c|c|}
\hline $\mathcal{G}$ & Subalg. & $\begin{array}{l}S l(2) \oplus U(1) \text { decompos. } \\
\text { (fundamental rep.) }\end{array}$ & $\begin{array}{l}\begin{array}{l}\text { Spin contents } \\
\text { (Hypercharge) }\end{array} \\
\end{array}$ & Gradation \\
\hline$A_{1}$ & $A_{1}$ & $\mathcal{D}_{1 / 2}$ & 2 & $\left(\frac{1}{2}, \frac{-1}{2}\right)$ \\
\hline \multirow[t]{2}{*}{$A_{2}$} & $A_{1}$ & $\mathcal{D}_{1 / 2}(y) \oplus \mathcal{D}_{0}(-2 y)$ & $\begin{array}{l}2, \frac{3}{2}, \frac{3}{2}, 1 \\
(0,3 y,-3 y, 0)\end{array}$ & $\begin{array}{l}\left(\frac{1}{2}, 0, \frac{-1}{2}\right) \\
\left(\frac{2}{3}, \frac{-1}{3}, \frac{-1}{3}\right)\end{array}$ \\
\hline & $A_{2}$ & $\mathcal{D}_{1}$ & 3,2 & $(1,0,-1)$ \\
\hline \multirow[t]{4}{*}{$B_{2}$} & $A_{1}$ & $2 \mathcal{D}_{1 / 2} \oplus \mathcal{D}_{0}$ & $2,2 * \frac{3}{2}, 3 * 1$ & $\left(\frac{1}{2}, \frac{1}{2}\right)$ \\
\hline & $\left.\begin{array}{c}A_{1}^{2} \\
2 A_{1}\end{array}\right\}$ & $\mathcal{D}_{1} \oplus \mathcal{D}_{0}(y) \oplus \mathcal{D}_{0}(-y)$ & $2,2,2,1$ & $(1,0)$ \\
\hline & & & $(0, y,-y, 0)$ & $\begin{array}{l}\left(1, \frac{1}{2}\right) \\
(1,1)\end{array}$ \\
\hline & $B_{2}$ & $\mathcal{D}_{2}$ & 4,2 & $(2,1)$ \\
\hline \multirow[t]{4}{*}{$C_{2}$} & $A_{1}$ & $\mathcal{D}_{1 / 2} \oplus 2 \mathcal{D}_{0}$ & $2,2 * \frac{3}{2}, 3 * 1$ & $\left(\frac{1}{2}, 0\right)$ \\
\hline & $\left.\begin{array}{c}2 A_{1} \\
A_{1}^{2}\end{array}\right\}$ & $\mathcal{D}_{1 / 2}(y) \oplus \mathcal{D}_{1 / 2}(-y)$ & $2,2,2,1$ & $\left(\frac{1}{2}, \frac{1}{2}\right)$ \\
\hline & & & $(0,2 y,-2 y, 0)$ & $\begin{array}{l}\left(\frac{3}{4}, \frac{1}{4}\right) \\
(1,0)\end{array}$ \\
\hline & $C_{2}$ & $\mathcal{D}_{3 / 2}$ & 4,2 & $\left(\frac{3}{2}, \frac{1}{2}\right)$ \\
\hline
\end{tabular}

Table 1: $W$ algebras for Lie algebras of rank 1 and 2 . 


\begin{tabular}{|c|c|c|c|c|}
\hline$\overline{\mathcal{G}}$ & Subalg. & $\begin{array}{l}S l(2) \oplus U(1) \text { decompos. } \\
\text { (fundamental rep.) }\end{array}$ & $\begin{array}{l}\text { Spin contents } \\
\text { (Hypercharge) }\end{array}$ & Gradation \\
\hline \multirow[t]{4}{*}{$A_{3}$} & $A_{1}$ & $\mathcal{D}_{1 / 2}(y) \oplus 2 \mathcal{D}_{0}(-y)$ & $\begin{array}{l}2,4 * \frac{3}{2}, 4 * 1 \\
(0,2 y, 2 y,-2 y,-2 y, 4 * 0)\end{array}$ & $\begin{array}{l}\left(\frac{1}{2}, 0,0, \frac{-1}{2}\right) \\
\left(\frac{3}{4}, \frac{-1}{4}, \frac{-1}{4}, \frac{-1}{4}\right)\end{array}$ \\
\hline & $2 A_{1}$ & $2 \mathcal{D}_{1 / 2}$ & $4 * 2,3 * 1$ & $\left(\frac{1}{2}, \frac{1}{2}, \frac{-1}{2}, \frac{-1}{2}\right)$ \\
\hline & $A_{2}$ & $\mathcal{D}_{1}(y) \oplus \mathcal{D}_{0}(-3 y)$ & $\begin{array}{l}3,2,2,2,1 \\
(0,2 y,-2 y, 0,0)\end{array}$ & $\begin{array}{l}(1,0,0,-1) \\
\left(\frac{5}{4}, \frac{1}{4}, \frac{-3}{4}, \frac{-3}{4}\right) \\
\left(\frac{9}{8}, \frac{1}{8}, \frac{-3}{8}, \frac{-7}{8}\right)\end{array}$ \\
\hline & $A_{3}$ & $\mathcal{D}_{3 / 2}$ & $4,3,2$ & $\left(\frac{3}{2}, \frac{1}{2}, \frac{-1}{2}, \frac{-3}{2}\right)$ \\
\hline \multirow[t]{4}{*}{$D_{3}$} & $A_{1}$ & $2 \mathcal{D}_{1 / 2} \oplus \mathcal{D}_{0}(y) \oplus \mathcal{D}_{0}(-y)$ & $\begin{array}{l}2,4 * \frac{3}{2}, 4 * 1 \\
(0, y, y,-y,-y, 4 * 0)\end{array}$ & $\begin{array}{l}\left(\frac{1}{2}, \frac{1}{2}, 0\right) \\
\left(\frac{1}{2}, \frac{1}{2}, \frac{1}{2}\right)\end{array}$ \\
\hline & $2 A_{1}$ & $\mathcal{D}_{1} \oplus 3 \mathcal{D}_{0}$ & $4 * 2,3 * 1$ & $(1,0,0)$ \\
\hline & $A_{2}$ & $\mathcal{D}_{1}(y) \oplus \mathcal{D}_{1}(-y)$ & $\begin{array}{l}3,2,2,2,1 \\
(0,2 y,-2 y, 0,0)\end{array}$ & $\begin{array}{l}(1,1,0) \\
\left(\frac{5}{4}, \frac{3}{4}, \frac{1}{4}\right) \\
\left(\frac{3}{2}, \frac{1}{2}, \frac{1}{2}\right)\end{array}$ \\
\hline & $D_{3}$ & $\mathcal{D}_{2} \oplus \mathcal{D}_{0}$ & $4,3,2$ & $(2,1,0)$ \\
\hline
\end{tabular}

Table 2: $W$ algebras for $A_{3} \equiv D_{3}$. 


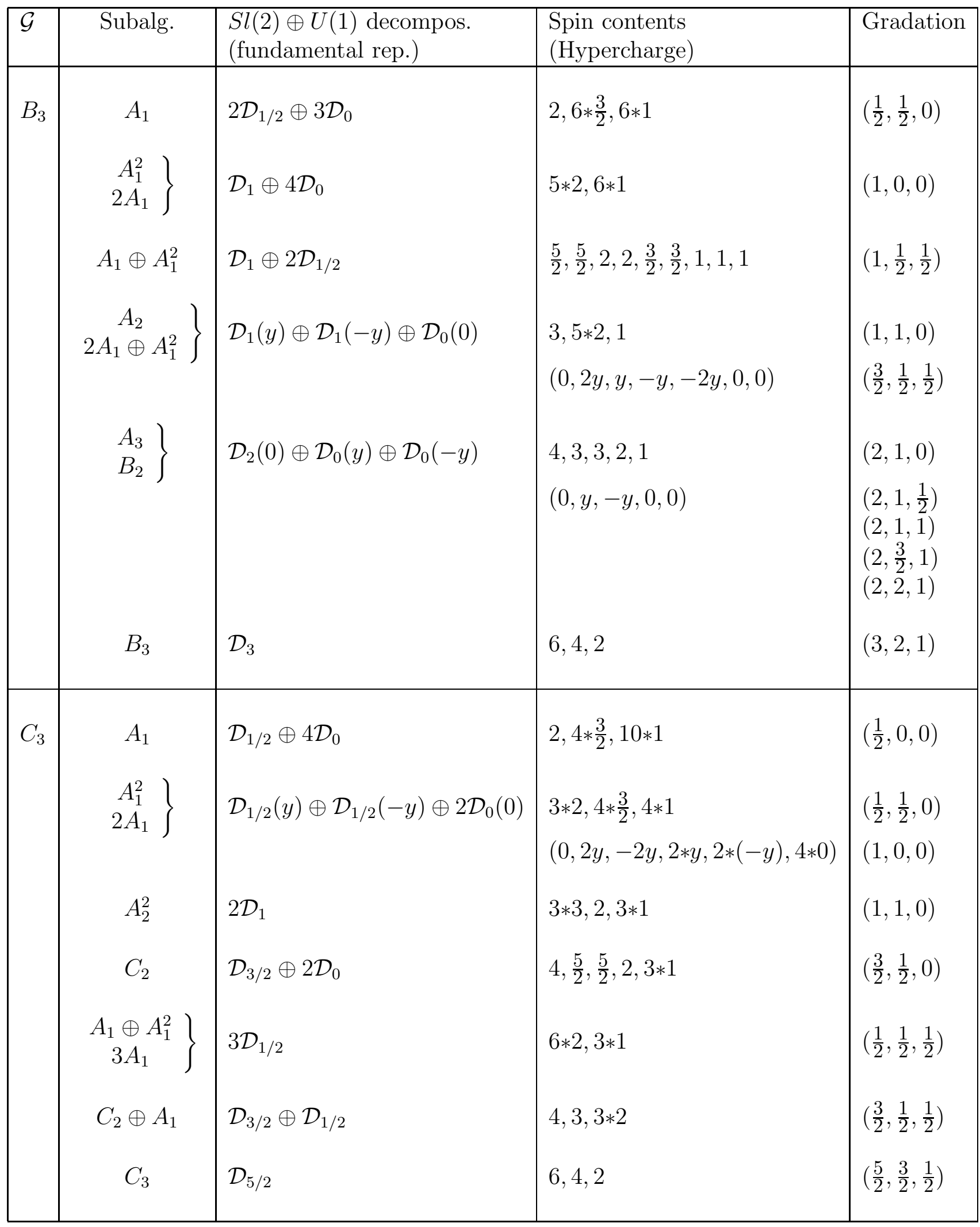

Table 3: $W$ algebras for $B_{3}$ and $C_{3}$. 


\begin{tabular}{|c|c|c|c|}
\hline Subalg. & $\begin{array}{l}S l(2) \oplus U(1) \text { decompos. } \\
\text { (fundamental rep.) }\end{array}$ & $\begin{array}{l}\text { Spin contents } \\
\text { (Hypercharge) }\end{array}$ & Gradation \\
\hline$A_{1}$ & $\mathcal{D}_{1 / 2}(y) \oplus 3 \mathcal{D}_{0}\left(\frac{-2 y}{3}\right)$ & $\begin{array}{l}2,6 * \frac{3}{2}, 9 * 1 \\
\left(0,3 * \frac{5 y}{3}, 3 * \frac{-5 y}{3}, 9 * 0\right)\end{array}$ & $\begin{array}{l}\left(\frac{1}{2}, 0,0,0, \frac{-1}{2}\right) \\
\left(\frac{4}{5}, \frac{-1}{5}, \frac{-1}{5}, \frac{-1}{5}, \frac{-1}{5}\right)\end{array}$ \\
\hline $2 A_{1}$ & $2 \mathcal{D}_{1 / 2}(y) \oplus \mathcal{D}_{0}(-4 y)$ & $\begin{array}{l}4 * 2,4 * \frac{3}{2}, 4 * 1 \\
(4 * 0,2 * 5 y, 2 *(-5 y), 4 * 0)\end{array}$ & $\begin{array}{l}\left(\frac{1}{2}, \frac{1}{2}, 0, \frac{-1}{2}, \frac{-1}{2}\right) \\
\left(\frac{3}{5}, \frac{3}{5}, \frac{-2}{5}, \frac{-2}{5}, \frac{-2}{5}\right)\end{array}$ \\
\hline$A_{2}$ & $\mathcal{D}_{1}(y) \oplus 2 \mathcal{D}_{0}\left(\frac{-3 y}{2}\right)$ & $\begin{array}{l}3,5 * 2,4 * 1 \\
\left(0,2 * \frac{5 y}{2}, 0,2 * \frac{-5 y}{2}, 4 * 0\right)\end{array}$ & $\begin{array}{l}(1,0,0,0,-1) \\
\left(\frac{6}{5}, \frac{1}{5}, \frac{-3}{10}, \frac{-3}{10}, \frac{-4}{5}\right) \\
\left(\frac{7}{5}, \frac{2}{5}, \frac{-3}{5}, \frac{-3}{5}, \frac{-3}{5}\right)\end{array}$ \\
\hline$A_{2} \oplus A_{1}$ & $\mathcal{D}_{1}(y) \oplus \mathcal{D}_{1 / 2}\left(\frac{-3 y}{2}\right)$ & $\begin{array}{l}3,2 * \frac{5}{2}, 2 * 2,2 * \frac{3}{2}, 1 \\
\left(0, \frac{5 y}{3}, \frac{-5 y}{3}, 0,0, \frac{5 y}{3}, \frac{-5 y}{3}, 0\right)\end{array}$ & $\begin{array}{l}\left(1, \frac{1}{2}, 0, \frac{-1}{2},-1\right) \\
\left(\frac{13}{20}, \frac{1}{20}, \frac{3}{10}, \frac{-19}{20}, \frac{-7}{10}\right)\end{array}$ \\
\hline$A_{3}$ & $\mathcal{D}_{3 / 2}(y) \oplus \mathcal{D}_{0}(-4 y)$ & $\begin{array}{l}4,3,2 * \frac{5}{2}, 2,1 \\
(0,0,5 y,-5 y, 2 * 0)\end{array}$ & $\begin{array}{l}\left(\frac{3}{2}, \frac{1}{2}, 0, \frac{-1}{2}, \frac{-3}{2}\right) \\
\left(\frac{9}{5}, \frac{4}{5}, \frac{-1}{5}, \frac{-6}{5}, \frac{-6}{5}\right) \\
\left(\frac{8}{5}, \frac{3}{5}, \frac{-2}{5}, \frac{-2}{5}, \frac{-7}{5}\right) \\
\left(\frac{17}{10}, \frac{7}{10}, \frac{-3}{10}, \frac{-4}{5}, \frac{-13}{10}\right)\end{array}$ \\
\hline$A_{4}$ & $\mathcal{D}_{2}$ & $5,4,3,2$ & $(2,1,0,-1,-2)$ \\
\hline
\end{tabular}

Table 4: $W$ algebras for $A_{4}$. 


\begin{tabular}{|c|c|c|c|}
\hline Subalg. & $\begin{array}{l}S l(2) \oplus U(1) \text { decompos. } \\
\text { (fundamental rep.) }\end{array}$ & $\begin{array}{l}\text { Spin contents } \\
\text { (Hypercharge) }\end{array}$ & Gradation \\
\hline$A_{1}$ & $2 \mathcal{D}_{1 / 2} \oplus 5 \mathcal{D}_{0}$ & $2,10 * \frac{3}{2}, 13 * 1$ & $\left(\frac{1}{2}, \frac{1}{2}, 0,0\right)$ \\
\hline $\left.\begin{array}{c}A_{1}^{2} \\
2 A_{1}\end{array}\right\}$ & $\mathcal{D}_{1} \oplus 6 \mathcal{D}_{0}$ & $7 * 2,15 * 1$ & $(1,0,0,0)$ \\
\hline$\left(2 A_{1}\right)^{\prime}$ & $4 \mathcal{D}_{1 / 2} \oplus \mathcal{D}_{0}$ & $6 * 2,4 * \frac{3}{2}, 10 * 1$ & $\left(\frac{1}{2}, \frac{1}{2}, \frac{1}{2}, \frac{1}{2}\right)$ \\
\hline $\left.\begin{array}{c}A_{1} \oplus A_{1}^{2} \\
3 A_{1}\end{array}\right\}$ & $\mathcal{D}_{1} \oplus 2 \mathcal{D}_{1 / 2} \oplus \mathcal{D}_{0}(y) \oplus \mathcal{D}_{0}(-y)$ & $\begin{array}{l}\frac{5}{2}, \frac{5}{2}, 4 * 2,6 * \frac{3}{2}, 6 * 1 \\
(0,0, y,-y, 0,0, y, y,-y,-y, 4 * 0)\end{array}$ & $\begin{array}{l}\left(1, \frac{1}{2}, \frac{1}{2}, 0\right) \\
\left(1, \frac{1}{2}, \frac{1}{2}, \frac{1}{2}\right)\end{array}$ \\
\hline $\left.\begin{array}{c}A_{2} \\
4 A_{1} \\
2 A_{1} \oplus A_{1}^{2}\end{array}\right\}$ & $\mathcal{D}_{1}(y) \oplus \mathcal{D}_{1}(-y) \oplus 3 \mathcal{D}_{0}(0)$ & $\begin{array}{l}3,9 * 2,4 * 1 \\
(0,3 * y, 3 *(-y), 2 y,-2 y, 5 * 0)\end{array}$ & $\begin{array}{l}(1,1,0,0) \\
\left(\frac{3}{2}, \frac{1}{2}, \frac{1}{2}, 0\right)\end{array}$ \\
\hline$A_{2} \oplus A_{1}^{2}$ & $3 \mathcal{D}_{1}$ & $3 * 3,6 * 2,3 * 1$ & $(1,1,1,0)$ \\
\hline$A_{3}$ & $2 \mathcal{D}_{3 / 2} \oplus \mathcal{D}_{0}$ & $4,3 * 3,2 * \frac{5}{2}, 2,3 * 1$ & $\left(\frac{3}{2}, \frac{3}{2}, \frac{1}{2}, \frac{1}{2}\right)$ \\
\hline $\left.\begin{array}{l}B_{2} \\
A_{3}\end{array}\right\}$ & $\mathcal{D}_{2} \oplus 4 \mathcal{D}_{0}$ & $4,4 * 3,2,6 * 1$ & $(2,1,0,0)$ \\
\hline$B_{2} \oplus A_{1}$ & $\mathcal{D}_{2} \oplus 2 \mathcal{D}_{1 / 2}$ & $4,2 * \frac{7}{2}, 2 * \frac{5}{2}, 2,2,3 * 1$ & $\left(2,1, \frac{1}{2}, \frac{1}{2}\right)$ \\
\hline $\left.\begin{array}{c}B_{2} \oplus 2 A_{1} \\
A_{3} \oplus A_{1}^{2}\end{array}\right\}$ & $\mathcal{D}_{2} \oplus \mathcal{D}_{1} \oplus \mathcal{D}_{0}$ & $4,4,3,3,4 * 2$ & $(2,1,1,0)$ \\
\hline $\left.\begin{array}{l}B_{3} \\
D_{4}\end{array}\right\}$ & $\mathcal{D}_{3}(0) \oplus \mathcal{D}_{0}(y) \oplus \mathcal{D}_{0}(-y)$ & $\begin{array}{l}6,3 * 4,2,1 \\
(0, y,-y, 3 * 0)\end{array}$ & $\begin{array}{l}(3,2,1,0) \\
(3,2,1, y)\end{array}$ \\
\hline$B_{4}$ & $\mathcal{D}_{4}$ & $8,6,4,2$ & $(4,3,2,1)$ \\
\hline
\end{tabular}

Table 5: $W$ algebras for $B_{4}$. 


\begin{tabular}{|c|c|c|c|}
\hline Subalg. & $\begin{array}{l}S l(2) \oplus U(1) \text { decompos. } \\
\text { (fundamental rep.) }\end{array}$ & \begin{tabular}{|l|}
$\begin{array}{l}\text { Spin contents } \\
\text { (Hypercharge) }\end{array}$ \\
\end{tabular} & Gradation \\
\hline$A_{1}$ & $\mathcal{D}_{1 / 2} \oplus 6 \mathcal{D}_{0}$ & $2,6 * \frac{3}{2}, 21 * 1$ & $\left(\frac{1}{2}, 0,0,0\right)$ \\
\hline $\left.\begin{array}{c}A_{1}^{2} \\
2 A_{1}\end{array}\right\}$ & $\mathcal{D}_{1 / 2}(y) \oplus \mathcal{D}_{1 / 2}(-y) \oplus 4 \mathcal{D}_{0}(0)$ & $\begin{array}{l}3 * 2,8 * \frac{3}{2}, 11 * 1 \\
(0,2 y,-2 y, 4 * y, 4 *(-y), 11 * 0)\end{array}$ & $\begin{array}{l}\left(\frac{1}{2}, \frac{1}{2}, 0,0\right) \\
(1,0,0,0)\end{array}$ \\
\hline $\left.\begin{array}{c}A_{1} \oplus A_{1}^{2} \\
3 A_{1}\end{array}\right\}$ & $3 \mathcal{D}_{1 / 2} \oplus 2 \mathcal{D}_{0}$ & $6 * 2,6 * \frac{3}{2}, 6 * 1$ & $\left(\frac{1}{2}, \frac{1}{2}, \frac{1}{2}, 0\right)$ \\
\hline $\left.\begin{array}{c}2 A_{1}^{2} \\
4 A_{1} \\
2 A_{1} \oplus A_{1}^{2}\end{array}\right\}$ & $4 \mathcal{D}_{1 / 2}$ & $10 * 2,6 * 1$ & $\left(\frac{1}{2}, \frac{1}{2}, \frac{1}{2}, \frac{1}{2}\right)$ \\
\hline$A_{2}^{2}$ & $2 \mathcal{D}_{1} \oplus 2 \mathcal{D}_{0}$ & $3,3,3,5 * 2,6 * 1$ & $(1,1,0,0)$ \\
\hline$A_{2}^{2} \oplus A_{1}$ & $2 \mathcal{D}_{1} \oplus \mathcal{D}_{1 / 2}$ & $3 * 3,2 * \frac{5}{2}, 2 * 2,2 * \frac{3}{2}, 3 * 1$ & $\left(1,1, \frac{1}{2}, 0\right)$ \\
\hline$C_{2}$ & $\mathcal{D}_{3 / 2} \oplus 4 \mathcal{D}_{0}$ & $4,4 * \frac{5}{2}, 2,10 * 1$ & $\left(\frac{3}{2}, \frac{1}{2}, 0,0\right)$ \\
\hline$C_{2} \oplus A_{1}$ & $\mathcal{D}_{3 / 2} \oplus \mathcal{D}_{1 / 2} \oplus 2 \mathcal{D}_{0}$ & $4,3,2 * \frac{5}{2}, 3 * 2,2 * \frac{3}{2}, 3 * 1$ & $\left(\frac{3}{2}, \frac{1}{2}, \frac{1}{2}, 0\right)$ \\
\hline $\left.\begin{array}{r}C_{2} \oplus A_{1}^{2} \\
C_{2} \oplus 2 A_{1}\end{array}\right\}$ & $\mathcal{D}_{3 / 2}(0) \oplus \mathcal{D}_{1 / 2}(y) \oplus \mathcal{D}_{1 / 2}(-y)$ & $\begin{array}{l}4,2 * 3,6 * 2,1 \\
(0, y,-y, 2 y,-2 y, y,-y, 3 * 0)\end{array}$ & $\begin{array}{l}\left(\frac{3}{2}, \frac{1}{2}, \frac{1}{2}, \frac{1}{2}\right) \\
\left(\frac{3}{2}, 1, \frac{1}{2}, 0\right)\end{array}$ \\
\hline $\left.\begin{array}{c}A_{3}^{2} \\
2 C_{2}\end{array}\right\}$ & $\mathcal{D}_{3 / 2}(y) \oplus \mathcal{D}_{3 / 2}(-y)$ & $\begin{array}{l}3 * 4,3,3 * 2,1 \\
(0,2 y,-2 y, 0,2 y,-2 y, 2 * 0)\end{array}$ & $\begin{array}{l}\left(\frac{3}{2}, \frac{3}{2}, \frac{1}{2}, \frac{1}{2}\right) \\
(2,1,1,0)\end{array}$ \\
\hline$C_{3}$ & $\mathcal{D}_{5 / 2} \oplus 2 \mathcal{D}_{0}$ & $6,4, \frac{7}{2}, \frac{7}{2}, 2,3 * 1$ & $\left(\frac{5}{2}, \frac{3}{2}, \frac{1}{2}, 0\right)$ \\
\hline$C_{3} \oplus A_{1}$ & $\mathcal{D}_{5 / 2} \oplus \mathcal{D}_{1 / 2}$ & $6,4,4,3,2,2$ & $\left(\frac{5}{2}, \frac{3}{2}, \frac{1}{2}, \frac{1}{2}\right)$ \\
\hline$C_{4}$ & $\mathcal{D}_{7 / 2}$ & $8,6,4,2$ & $\left(\frac{7}{2}, \frac{5}{2}, \frac{3}{2}, \frac{1}{2}\right)$ \\
\hline
\end{tabular}

Table 6: $W$ algebras for $C_{4}$. 


\begin{tabular}{|c|l|l|l|}
\hline Subalg. & $\begin{array}{l}S l(2) \oplus U(1) \text { decompos. } \\
\text { (fundamental rep.) }\end{array}$ & $\begin{array}{l}\text { Spin contents } \\
\text { (Hypercharge) }\end{array}$ & Gradation \\
\hline$A_{1}$ & $2 \mathcal{D}_{1 / 2} \oplus 4 \mathcal{D}_{0}$ & $2,8 * \frac{3}{2}, 9 * 1$ & $\left(\frac{1}{2}, \frac{1}{2}, 0,0\right)$ \\
$2 A_{1}$ & $\mathcal{D}_{1} \oplus 5 \mathcal{D}_{0}$ & $6 * 2,10 * 1$ & $(1,0,0,0)$ \\
$\left(2 A_{1}\right)^{\prime}$ & $4 \mathcal{D}_{1 / 2}$ & $6 * 2,10 * 1$ & $\left(\frac{1}{2}, \frac{1}{2}, \frac{1}{2}, \frac{1}{2}\right)$ \\
$3 A_{1}$ & $\mathcal{D}_{1} \oplus 2 \mathcal{D}_{1 / 2} \oplus \mathcal{D}_{0}$ & $\frac{5}{2}, \frac{5}{2}, 3 * 2,4 * \frac{3}{2}, 3 * 1$ & $\left(1, \frac{1}{2}, \frac{1}{2}, 0\right)$ \\
$A_{2}$ & $\mathcal{D}_{1}\left(y_{1}\right) \oplus \mathcal{D}_{1}\left(-y_{1}\right) \oplus$ & $3,7 * 2,2 * 1$ & $(1,1,0,0)$ \\
$4 A_{1}$ & $\oplus \mathcal{D}_{0}\left(y_{0}\right) \oplus \mathcal{D}_{0}\left(-y_{0}\right)$ & $\left(0, \pm y_{1} \pm y_{0}, \pm 2 y_{1}, 3 * 0\right)$ & $\left(\frac{3}{2}, \frac{1}{2}, \frac{1}{2}, 0\right)$ \\
& & & $(2,1,0,0)$ \\
& & $\left(1,1, \frac{1}{2}, 0\right)$ \\
& & $4,3 * 3,2,3 * 1$ & $\left(\frac{3}{2}, \frac{1}{2}, \frac{1}{2}, \frac{1}{2}\right)$ \\
$A_{3}$ & $2 \mathcal{D}_{3 / 2}$ & $\left(\frac{3}{2}, \frac{3}{2}, \frac{1}{2}, \frac{1}{2}\right)$ \\
$D_{3}$ & $\mathcal{D}_{2} \oplus 3 \mathcal{D}_{0}$ & $(2,1,0,0)$ \\
$B_{2} \oplus B_{1}$ & $\mathcal{D}_{2} \oplus \mathcal{D}_{1}$ & $4,3 * 3,2,3 * 1$ & $(2,1,1,0)$ \\
$D_{4}$ & $\mathcal{D}_{3} \oplus \mathcal{D}_{0}$ & $(3,2,1,0)$ \\
\hline
\end{tabular}

Table 7: $W$ algebras for $D_{4}$.

\begin{tabular}{|c|l|l|l|}
\hline $\begin{array}{c}\text { Minimal including } \\
\text { Regular Subalgebra }\end{array}$ & $\begin{array}{l}S l(2) \text { decomposition } \\
\text { (fundamental rep.) }\end{array}$ & Spin contents & $\begin{array}{l}\text { Defining } \\
\text { Vector }\end{array}$ \\
\hline$A_{1}$ & $2 \mathcal{D}_{1 / 2} \oplus 3 \mathcal{D}_{0}$ & $2,4 * \frac{3}{2}, 1,1,1$ & $\left(\frac{1}{2}, \frac{1}{2}, 0\right)$ \\
$A_{1}^{2}$ & $\mathcal{D}_{1} \oplus 2 \mathcal{D}_{1 / 2}$ & $\frac{5}{2}, \frac{5}{2}, 2,1,1,1$ & $\left(1, \frac{1}{2}, 0\right)$ \\
$A_{1} \oplus A_{1}^{2}$ & $2 \mathcal{D}_{1} \oplus \mathcal{D}_{0}$ & $3,2,2,2$ & $(1,0,0)$ \\
$G_{2}$ & $\mathcal{D}_{3}$ & 6,2 & $\left(2, \frac{3}{2}, \frac{1}{2}\right)$ \\
\hline
\end{tabular}

Table 8: Classification for $G_{2}$. 


\begin{tabular}{|c|c|c|}
\hline $\begin{array}{l}\text { Minimal including } \\
\text { Regular Subalgebra }\end{array}$ & $\begin{array}{l}S l(2) \text { decomposition } \\
\text { (fundamental rep.) }\end{array}$ & Spin contents \\
\hline$A_{1}$ & $6 \mathcal{D}_{1 / 2} \oplus 14 \mathcal{D}_{0}$ & $2,14 * \frac{3}{2}, 21 * 1$ \\
\hline $\left.\begin{array}{c}A_{1}^{2} \\
2 A_{1}\end{array}\right\}$ & $\mathcal{D}_{1} \oplus 8 \mathcal{D}_{1 / 2} \oplus 7 \mathcal{D}_{0}$ & $7 * 2,10 * \frac{3}{2}, 15 * 1$ \\
\hline $\left.\begin{array}{c}A_{1} \oplus A_{1}^{2} \\
3 A_{1}\end{array}\right\}$ & $3 \mathcal{D}_{1} \oplus 6 \mathcal{D}_{1 / 2} \oplus 5 \mathcal{D}_{0}$ & $2 * \frac{5}{2}, 6 * 2,10 * \frac{3}{2}, 6 * 1$ \\
\hline $\left.\begin{array}{c}4 A_{1} \\
2 A_{1} \oplus A_{1}^{2} \\
A_{2}\end{array}\right\}$ & $6 \mathcal{D}_{1} \oplus 8 \mathcal{D}_{0}$ & $3,13 * 2,8 * 1$ \\
\hline$A_{2}^{2}$ & $\mathcal{D}_{2} \oplus 7 \mathcal{D}_{1}$ & $7 * 3,2,14 * 1$ \\
\hline$A_{2} \oplus A_{1}^{2}$ & $\mathcal{D}_{2} \oplus 2 \mathcal{D}_{3 / 2} \oplus 3 \mathcal{D}_{1} \oplus 2 \mathcal{D}_{1 / 2}$ & $2 * 4,3 * 3,6 * 2,2 * \frac{3}{2}, 1$ \\
\hline$A_{1} \oplus A_{2}^{2}$ & $2 \mathcal{D}_{3 / 2} \oplus 3 \mathcal{D}_{1} \oplus 4 \mathcal{D}_{1 / 2} \oplus \mathcal{D}_{0}$ & $3 * 3,2 * \frac{5}{2}, 6 * 2,4 * \frac{3}{2}, 3 * 1$ \\
\hline $\left.\begin{array}{c}A_{2}^{2} \oplus A_{2} \\
A_{3} \oplus A_{1}^{2} \\
B_{2} \oplus A_{1}^{2} \\
B_{2} \oplus 2 A_{1}\end{array}\right\}$ & $3 \mathcal{D}_{2} \oplus 3 \mathcal{D}_{1} \oplus 2 \mathcal{D}_{0}$ & $2 * 4,4 * 3,6 * 2$ \\
\hline $\left.\begin{array}{l}B_{2} \\
A_{3}\end{array}\right\}$ & $\mathcal{D}_{2} \oplus 4 \mathcal{D}_{3 / 2} \oplus 5 \mathcal{D}_{0}$ & $4,4 * 3,4 * \frac{5}{2}, 2,6 * 1$ \\
\hline$B_{2} \oplus A_{1}$ & $2 \mathcal{D}_{2} \oplus 2 \mathcal{D}_{3 / 2} \oplus \mathcal{D}_{1} \oplus 2 \mathcal{D}_{1 / 2} \oplus \mathcal{D}_{0}$ & $4,2 * \frac{7}{2}, 3,4 * \frac{5}{2}, 3 * 2,3 * 1$ \\
\hline $\left.\begin{array}{l}B_{3} \\
D_{4}\end{array}\right\}$ & $3 \mathcal{D}_{3} \oplus 5 \mathcal{D}_{0}$ & $6,5 * 4,2,3 * 1$ \\
\hline$B_{4}$ & $\mathcal{D}_{5} \oplus \mathcal{D}_{4} \oplus \mathcal{D}_{2} \oplus \mathcal{D}_{0}$ & $8,2 * 6,4,3,2$ \\
\hline$C_{3}$ & $\mathcal{D}_{4} \oplus 2 \mathcal{D}_{5 / 2} \oplus \mathcal{D}_{2}$ & $6,2 * \frac{11}{2}, 4,2 * \frac{5}{2}, 2,3 * 1$ \\
\hline$C_{3} \oplus A_{1}$ & $\mathcal{D}_{4} \oplus \mathcal{D}_{3} \oplus 2 \mathcal{D}_{2}$ & $2 * 6,5,4,3,3 * 2$ \\
\hline$F_{4}$ & $\mathcal{D}_{8} \oplus \mathcal{D}_{4}$ & $12,8,6,2$ \\
\hline
\end{tabular}

Table 9: Classification for $F_{4}$. 


\section{Part II}

\section{Super $W$ algebras built on Lie superalgebras}

\section{The $O S p(1 \mid 2)$ subsuperalgebras of simple Lie superalgebras}

The determination of the different $O S p(1 \mid 2)$ subalgebras in a simple Lie superalgebra $\mathcal{G}=\mathcal{G}_{B} \oplus \mathcal{G}_{F}$ is greatly simplified by the two following remarks:

1) The $S l(2)$ part of $O S p(1 \mid 2)$ is in the (semi)simple bosonic part of the considered superalgebra. The knowledge of a method to classify the $S l(2)$ subalgebras of a simple Lie algebra can be obviously generalized to the case of a direct sum of two (or three, cf. $D(2,1 ; \alpha)$ ) simple algebras.

2) Any representation of $O S p(1 \mid 2)$ is completely irreducible, and any irreducible $O S p(1 \mid 2)$ representation $\mathcal{R}_{j}$ ( $j$ integer or half-integer) is the direct sum of two $S l(2)$ representations $\mathcal{D}_{j} \oplus \mathcal{D}_{j-1 / 2}$ with an exception for the trivial one-dimensional representation $\mathcal{R}_{0}=\mathcal{D}_{0}$. From the reduction of the fundamental representation of $\mathcal{G}$ into $S l(2)$ ones, it is therefore easy to verify whether the $S l(2)$ under consideration can be embedded into an $O S p(1 \mid 2)$ superalgebra.

Now, in the same way that the $S l(2)$ subalgebras of a simple Lie algebra $\mathcal{G}$ are principal subalgebras of the $\mathcal{G}$ regular subalgebras (up to exceptions arising in the $D_{n}$ case, see Section (3), it is rather clear that the $O S p(1 \mid 2)$ subsuperalgebras of a simple Lie superalgebra $\mathcal{G}$ are superprincipal in the $\mathcal{G}$ regular subsuperalgebras (up to exceptions arising in the $D(m, n)$ case). One recalls that the definition of a regular subsuperalgebra (SSA) is a direct generalization of that of an algebra (see 3.3), and such SSA can be obtained from the extended Dynkin diagrams for superalgebras, as for simple algebras [18. Of course, since several Dynkin diagrams can be in general associated to the same superalgebra, one has to apply the method to each allowed Dynkin diagram specifying the superalgebra. A SSA of $\mathcal{G}$ which is not regular is called singular. An example of singular SSA of $\mathcal{G}$ is the superprincipal $O S p(1 \mid 2)$, when it exists. It is defined as

$$
\begin{aligned}
& F_{+}=\sum_{\alpha \in \Delta} E_{\alpha} \text { and } \quad F_{-}=\sum_{\alpha \in \Delta} E_{-\alpha} \\
& E_{+}=\left\{F_{+}, F_{+}\right\} \quad, \quad E_{-}=\left\{F_{-}, F_{-}\right\} \quad \text { and } H=\left\{F_{+}, F_{-}\right\}
\end{aligned}
$$

where $\Delta$ is a simple root system of $\mathcal{G}$.

Not all the simple Lie superalgebras admit a superprincipal embedding. Actually, it is clear from the expression of the $O S p(1 \mid 2)$ generators, that a superprincipal 
embedding can be defined only if the superalgebra under consideration has a completely fermionic simple root system $\Delta$ (which corresponds to a Dynkin diagram with only grey or/and black dots). Notice that this condition is necessary but not sufficient (the superalgebra $P S l(n \mid n)$ does not admit a superprincipal embedding although it has a completely fermionic simple root system). The simple superalgebras admitting a superprincipal $O S p(1 \mid 2)$ are the following: $S l(n+1 \mid n)$, $S l(n \mid n+1), O S p(2 n \pm 1 \mid 2 n), O S p(2 n \mid 2 n), O S p(2 n+2 \mid 2 n)$ with $n \geq 1$ and $D(2,1 ; \alpha)$ with $\alpha \neq 0, \pm 1$.

Finally, the method for classifying the $O S p(1 \mid 2)$ SSAs in a simple Lie superalgebra $\mathcal{G}$ can be summarized as follows:

Any $O S p(1 \mid 2) S S A$ in a simple Lie superalgebra $\mathcal{G}$ can be considered as the superprincipal $O S p(1 \mid 2) S S A$ of a regular $S S A \widetilde{\mathcal{G}}$ of $\mathcal{G}$, up to the following exceptions:

i) For $\mathcal{G}=O S p(2 n \pm 2 \mid 2 n)$ with $n \geq 2$, besides the superprincipal $O S p(1 \mid 2)$ $S S A s$ described above, there exist $O S p(1 \mid 2) S S A s$ associated to the singular embeddings $O S p(2 k \pm 1 \mid 2 k) \oplus O S p(2 n-2 k \pm 1 \mid 2 n-2 k)$ with $1 \leq k \leq n-1$.

ii) For $\mathcal{G}=O S p(2 n \mid 2 n)$ with $n \geq 2$, besides the $O S p(1 \mid 2)$ superprincipal embedding, there exist $O S p(1 \mid 2) S S A s$ associated to the singular embeddings $O S p(2 k \pm 1 \mid 2 k) \oplus O S p(2 n-2 k \mp 1 \mid 2 n-2 k) \subset O S p(2 n \mid 2 n)$ with $1 \leq k \leq n-1$.

\section{$10 O S p(1 \mid 2)$ decompositions of simple Lie su- peralgebras}

Following the general method explained above, once the possible $O S p(1 \mid 2)$ embeddings are determined in the simple Lie superalgebra $\mathcal{G}$, one has to reduce the adjoint representation of $\mathcal{G}$ into $O S p(1 \mid 2)$ supermultiplets. Consider an $O S p(1 \mid 2)$ SSA of $\mathcal{G}$, and let $\widetilde{\mathcal{G}}$ be the minimal including regular SSA of $\mathcal{G}$ having this $O S p(1 \mid 2)$ as superprincipal embedding. We will show on the example of $S l(m \mid n)$ how to obtain the decomposition of a simple Lie superalgebra starting from the decompositions of its bosonic and fermionic parts with respect to the bosonic $S l(2)$ subalgebra of the $O S p(1 \mid 2)$ under consideration. Moreover, we will see that such a decomposition can be obtained in a systematic way from the decomposition of the fundamental representation of the superalgebra with respect to the $O S p(1 \mid 2)$.

\subsection{The unitary superalgebras $S l(m \mid n)$}

The bosonic part of $\mathcal{G}=S l(m \mid n)$ with $m \neq n$ is $\mathcal{G}_{B}=S l(m) \oplus S l(n) \oplus U(1)$ and the fermionic part $\mathcal{G}_{F}$ is the $(\underline{m}, \bar{n}) \oplus(\bar{m}, \underline{n})$ representation of $\operatorname{Sl}(m) \oplus \operatorname{Sl}(n)$. 
The regular SSAs of $S l(m \mid n)$ which admit a superprincipal embedding are of the $S l(p+1 \mid p)$ or $S l(p \mid p+1)$ type.

Consider an $O S p(1 \mid 2)$ SSA of $\mathcal{G}$ such that the minimal including regular SSA in $\mathcal{G}$ is $\widetilde{\mathcal{G}}=S l(p+1 \mid p)$ with $p \leq \inf (m-1, n)$. Under $S l(2)$ (of $O S p(1 \mid 2)$ ), the representations $\underline{m}$ and $\underline{n}$ of $S l(m)$ and $S l(n)$ decompose as

$$
\begin{aligned}
& \underline{m}=\mathcal{D}_{p / 2} \oplus(m-p-1) \mathcal{D}_{0} \\
& \underline{n}=\mathcal{D}_{(p-1) / 2} \oplus(n-p) \mathcal{D}_{0}
\end{aligned}
$$

Therefore the fermionic part $\mathcal{G}_{F}$ reduces to

$$
\begin{aligned}
(\underline{m}, \bar{n}) \oplus(\bar{m}, \underline{n})= & 2\left(\mathcal{D}_{p / 2} \oplus(m-p-1) \mathcal{D}_{0}\right) \times\left(\mathcal{D}_{(p-1) / 2} \oplus(n-p) \mathcal{D}_{0}\right) \\
= & 2 \mathcal{D}_{p-1 / 2} \oplus 2 \mathcal{D}_{p-3 / 2} \oplus \ldots \oplus 2 \mathcal{D}_{1 / 2} \oplus 2(m-p-1) \mathcal{D}_{(p-1) / 2} \\
& \oplus 2(n-p) \mathcal{D}_{p / 2} \oplus 2(m-p-1)(n-p) \mathcal{D}_{0}
\end{aligned}
$$

The bosonic part $\mathcal{G}_{B}$ is decomposed as

$$
\begin{aligned}
\mathcal{G}_{B}= & S l(m) \oplus S l(n) \oplus U(1) \\
= & \left(\mathcal{D}_{p / 2} \oplus(m-p-1) \mathcal{D}_{0}\right) \times\left(\mathcal{D}_{p / 2} \oplus(m-p-1) \mathcal{D}_{0}\right) \\
& \oplus\left(\mathcal{D}_{(p-1) / 2} \oplus(n-p) \mathcal{D}_{0}\right) \times\left(\mathcal{D}_{(p-1) / 2} \oplus(n-p) \mathcal{D}_{0}\right)-\mathcal{D}_{0} \\
= & \mathcal{D}_{p} \oplus 2 \mathcal{D}_{p-1} \oplus \ldots \oplus 2 \mathcal{D}_{1} \oplus 2(m-p-1) \mathcal{D}_{p / 2} \\
& \oplus 2(n-p) \mathcal{D}_{(p-1) / 2} \oplus\left[(m-p-1)^{2}+(n-p)^{2}+1\right] \mathcal{D}_{0}
\end{aligned}
$$

Gathering the $S l(2)$ representations $\mathcal{D}_{j}$ into $O S p(1 \mid 2)$ irreducible representations $\mathcal{R}_{j}$, one finds that the adjoint representation of $S l(m \mid n)$ decomposes under the superprincipal $O S p(1 \mid 2)$ of $S l(p+1 \mid p) \subset S l(m \mid n)$ ast:

$$
\begin{aligned}
\frac{\mathbf{A d}[S l(m \mid n)]}{S l(p+1 \mid p)}= & \mathcal{R}_{p} \oplus \mathcal{R}_{p-1 / 2} \oplus \mathcal{R}_{p-1} \oplus \ldots \oplus \mathcal{R}_{1 / 2} \oplus 2(n-p) \mathcal{R}_{p / 2} \oplus 2(m-p-1) \mathcal{R}_{p / 2}^{\prime} \\
& \oplus\left[(m-p-1)^{2}+(n-p)^{2}\right] \mathcal{R}_{0} \oplus 2(m-p-1)(n-p) \mathcal{R}_{0}^{\prime}
\end{aligned}
$$

Notice that the $W_{j+1 / 2}$ superfield corresponding to the representation $\mathcal{R}_{j}=\mathcal{D}_{j} \oplus$ $\mathcal{D}_{j-1 / 2}$ has two component fields $w_{j+1}$ and $w_{j+1 / 2}$ of spins $j+1$ and $j+1 / 2$ respectively. If the representation $\mathcal{D}_{j}$ comes from the bosonic (resp. fermionic) part, $w_{j+1}$ is commuting (resp. anticommuting), whereas $w_{j+1 / 2}$ is anticommuting (resp. commuting). Therefore, if $j$ is integer, the generators $w_{j+1}$ and $w_{j+1 / 2}$ have the "right" statistics, whereas they have the "wrong" statistics if $j$ is half-integer. The representations $\mathcal{R}_{j}$ denoted with a prime are used in the case of $W$ superfields obeying to the "wrong" statistics.

\footnotetext{
${ }^{4}$ In the following, we will use $\frac{\operatorname{Ad}[\mathcal{G}]}{\widetilde{\mathcal{G}}}$ to denote the decomposition of the adjoint representation of $\mathcal{G}$ with respect to the superprincipal $\operatorname{OSp}(1 \mid 2)$ of $\widetilde{\mathcal{G}} \subset \mathcal{G}$.
} 
Actually, this decomposition (which was obtained above in a rather heavy way) can be derived directly from the decomposition of the fundamental representation of the superalgebra $S l(m \mid n)$ with respect to the $O S p(1 \mid 2)$ under consideration. From (10.1), the fundamental representation of $S l(m \mid n)$, of dimension $m+n$, decomposes as

$$
\underline{m+n}=\mathcal{R}_{p / 2} \oplus(m-p-1) \mathcal{R}_{0} \oplus(n-p) \mathcal{R}_{0}^{\pi}
$$

where we have introduced two kinds of $O S p(1 \mid 2)$ representations. An $O S p(1 \mid 2)$ representation is denoted $\mathcal{R}_{j}$ if the representation $D_{j}$ comes from the decomposition of the fundamental of $S l(m)$ and $\mathcal{R}_{j}^{\pi}$ if $D_{j}$ comes from the decomposition of the fundamental of $S l(n)$.

Then the adjoint representation of $S l(m \mid n)$ is obtained from the fundamental one by

$$
\operatorname{Ad}[S l(m \mid n)]=(\underline{m+n}) \times(\overline{m+n})-\underline{1}
$$

Using the general formula giving the product of two $O S p(1 \mid 2)$ representations $\mathcal{R}_{q_{1}}$ and $\mathcal{R}_{q_{2}}$ :

$$
\mathcal{R}_{q_{1}} \times \mathcal{R}_{q_{2}}=\bigoplus_{q=\left|q_{1}-q_{2}\right|}^{q=q_{1}+q_{2}} \mathcal{R}_{q} \quad \text { with } q \text { integer and half-integer }
$$

one recovers the decomposition of the adjoint representation of $S l(m \mid n)$ under the superprincipal $O S p(1 \mid 2)$ of $S l(p+1 \mid p)$ given by (10.4).

Now, we consider the $O S p(1 \mid 2)$ superprincipal embedding of $S l(p \mid p+1)$ in $\mathcal{G}$ with $p \leq \inf (m, n-1)$. Then the decompositions of the representations $\underline{m}$ and $\underline{n}$ of $\operatorname{Sl}(m)$ and $S l(n)$ are:

$$
\begin{aligned}
& \underline{m}=\mathcal{D}_{(p-1) / 2} \oplus(m-p) \mathcal{D}_{0} \\
& \underline{n}=\mathcal{D}_{p / 2} \oplus(n-p-1) \mathcal{D}_{0}
\end{aligned}
$$

leading to the following decomposition of the fundamental representation $\underline{m+n}$ of $S l(m \mid n)$ :

$$
\underline{m+n}=\mathcal{R}_{p / 2}^{\pi} \oplus(m-p) \mathcal{R}_{0} \oplus(n-p-1) \mathcal{R}_{0}^{\pi}
$$

Therefore, the decomposition of the adjoint representation reads

$$
\begin{aligned}
\frac{\operatorname{Ad}[S l(m \mid n)]}{S l(p \mid p+1)}= & \mathcal{R}_{p} \oplus \mathcal{R}_{p-1 / 2} \oplus \mathcal{R}_{p-1} \oplus \ldots \oplus \mathcal{R}_{1 / 2} \oplus 2(m-p) \mathcal{R}_{p / 2} \oplus 2(n-p-1) \mathcal{R}_{p / 2}^{\prime} \\
& \oplus\left[(m-p)^{2}+(n-p-1)^{2}\right] \mathcal{R}_{0} \oplus 2(m-p)(n-p-1) \mathcal{R}_{0}^{\prime}
\end{aligned}
$$


More generally, if $\widetilde{\mathcal{G}}$ is a sum of SSAs of $S l(p+1 \mid p)$ or $S l(p \mid p+1)$ type, each factor $S l(p+1 \mid p)$ gives rise to an $O S p(1 \mid 2)$ representation $\mathcal{R}_{p / 2}$ and each factor $S l(p \mid p+1)$ to an $O S p(1 \mid 2)$ representation $\mathcal{R}_{p / 2}^{\pi}$ in the decomposition of the fundamental $\underline{m+n}$ of $S l(m \mid n)$, completed eventually by singlets $\mathcal{R}_{0}$ or $\mathcal{R}_{0}^{\pi}$. Then the decomposition of the adjoint representation of $S l(m \mid n)$ is obtained by applying (10.6).

Finally, let us consider the case of the superalgebra $\operatorname{PSl}(n \mid n)$ whose bosonic part is $S l(n) \oplus S l(n)$ and its fermionic part is the $(\underline{n}, \bar{n}) \oplus(\bar{n}, \underline{n})$ representation of the bosonic subalgebra. If the minimal including regular SSA is $S l(p+1 \mid p)$ with $p \leq n-1$, the fundamental representation of $P S l(n \mid n)$ decomposes as

$$
\underline{2 n}=\mathcal{R}_{p / 2} \oplus(n-p-1) \mathcal{R}_{0} \oplus(n-p) \mathcal{R}_{0}^{\pi}
$$

and the adjoint representation of $\operatorname{PSl}(n \mid n)$ is given by

$$
\operatorname{Ad}[P S l(n \mid n)]=(\underline{2 n}) \times(\overline{2 n})-2 \underline{1}
$$

One finds therefore

$$
\begin{aligned}
\frac{\operatorname{Ad}[P S l(n \mid n)]}{S l(p+1 \mid p)}= & \mathcal{R}_{p} \oplus \mathcal{R}_{p-1 / 2} \oplus \mathcal{R}_{p-1} \oplus \ldots \oplus \mathcal{R}_{1 / 2} \oplus 2(n-p) \mathcal{R}_{p / 2} \oplus 2(n-p-1) \mathcal{R}_{p / 2}^{\prime} \\
& \oplus\left[(n-p-1)^{2}+(n-p)^{2}-1\right] \mathcal{R}_{0} \oplus 2(n-p-1)(n-p) \mathcal{R}_{0}^{\prime}
\end{aligned}
$$

The computation is completely analogous if the minimal including regular SSA is $S l(p \mid p+1)$.

\subsection{The orthosymplectic superalgebras $O S p(M \mid 2 n)$}

\subsubsection{Products of $O S p(1 \mid 2)$ irreducible representations}

Consider an $O S p(1 \mid 2) \mathrm{SSA}$ of $\mathcal{G}=O S p(M \mid 2 n)$ and let $\widetilde{\mathcal{G}}$ be the minimal including SSA in $\mathcal{G}$. Under the superprincipal $O S p(1 \mid 2)$ of $\widetilde{\mathcal{G}}$, the fundamental representation of $\mathcal{G}$, of dimension $M+2 n$, decomposes in a sum of $O S p(1 \mid 2)$ representations, generically denoted as

$$
\underline{M+2 n}=\left(\bigoplus_{j} \mathcal{R}_{j}\right) \oplus\left(\bigoplus_{j^{\prime}} \mathcal{R}_{j^{\prime}}^{\pi}\right)
$$

where the representations $\mathcal{R}_{j}$ and $\mathcal{R}_{j^{\prime}}^{\pi}$ have the same meaning as in the previous section : a representation $\mathcal{R}_{j}$ (resp. $\mathcal{R}_{j^{\prime}}^{\pi}$ ) corresponds here to an $O S p(1 \mid 2)$ representation where the $\mathcal{D}_{j}$ comes from the decomposition of the $S O(M)$ (resp. $S p(2 n))$ part.

In order to know how to obtain the decomposition of the adjoint representation of $O S p(M \mid 2 n)$ from the decomposition of the fundamental one, we come back for 
a while to the Abelian case [10], specializing for the moment to the superalgebra $O S p(2 m+1 \mid 2 m)$. In that case, the fundamental representation of $O S p(2 m+1 \mid 2 m)$ of dimension $4 m+1$ decomposes under its superprincipal $O S p(1 \mid 2)$ as

$$
\underline{4 m+1}=\mathcal{R}_{m}
$$

and thus the adjoint representation of $O S p(2 m+1 \mid 2 m)$ decomposes as

$$
\operatorname{Ad}[O S p(2 m+1 \mid 2 m)]=\left(\mathcal{D}_{m} \times \mathcal{D}_{m}\right)_{A} \oplus\left(\mathcal{D}_{m-1 / 2} \times \mathcal{D}_{m-1 / 2}\right)_{S} \oplus\left(\mathcal{D}_{m} \times \mathcal{D}_{m-1 / 2}\right)
$$

The two first terms correspond to the adjoint representations of $S O(2 m+1)$ and $S p(2 m)$ respectively, and the last one to the fermionic representation $(\underline{2 m+1}, \underline{2 m})$ of the bosonic part. Therefore, one has

$$
\begin{aligned}
\operatorname{Ad}[\operatorname{OSp}(2 m+1 \mid 2 m)] & =\left(\bigoplus_{k=1}^{m} \mathcal{D}_{2 k-1}\right) \oplus\left(\bigoplus_{k=1}^{m} \mathcal{D}_{2 k-1}\right) \oplus\left(\bigoplus_{k=1 / 2}^{2 m-1 / 2} \mathcal{D}_{k}\right) \\
& =\left(\bigoplus_{k=1}^{m} \mathcal{D}_{2 k-1} \oplus \mathcal{D}_{2 k-3 / 2}\right) \oplus\left(\bigoplus_{k=1}^{m} \mathcal{D}_{2 k-1 / 2} \oplus \mathcal{D}_{2 k-1}\right) \\
& =\bigoplus_{k=1}^{m}\left(\mathcal{R}_{2 k-1} \oplus \mathcal{R}_{2 k-1 / 2}\right)
\end{aligned}
$$

By analogy with the bosonic $S O(2 m)$ case (cf. 4.5), we set (with $m$ integer)

$$
\left(\mathcal{R}_{m} \times \mathcal{R}_{m}\right)_{A}=\bigoplus_{k=1}^{m}\left(\mathcal{R}_{2 k-1} \oplus \mathcal{R}_{2 k-1 / 2}\right) \quad \text { with } \quad k \in \mathbb{Z}
$$

Now we specialize to the superalgebra $O S p(2 m-1 \mid 2 m)$. In that case, the fundamental representation of $O S p(2 m-1 \mid 2 m)$ of dimension $4 m-1$ decomposes under its superprincipal $O S p(1 \mid 2)$ as

$$
\underline{4 m-1}=\mathcal{R}_{m-1 / 2}^{\pi}
$$

and thus the adjoint representation of $O S p(2 m-1 \mid 2 m)$ decomposes as

$$
\operatorname{Ad}[O S p(2 m-1 \mid 2 m)]=\left(\mathcal{D}_{m-1} \times \mathcal{D}_{m-1}\right)_{A} \oplus\left(\mathcal{D}_{m-1 / 2} \times \mathcal{D}_{m-1 / 2}\right)_{S} \oplus\left(\mathcal{D}_{m-1} \times \mathcal{D}_{m-1 / 2}\right)
$$

Therefore, one has

$$
\begin{aligned}
\operatorname{Ad}[\operatorname{OSp}(2 m-1 \mid 2 m)] & =\left(\bigoplus_{k=1}^{m-1} \mathcal{D}_{2 k-1}\right) \oplus\left(\bigoplus_{k=1}^{m} \mathcal{D}_{2 k-1}\right) \oplus\left(\bigoplus_{k=1 / 2}^{2 m-3 / 2} \mathcal{D}_{k}\right) \\
& =\left(\bigoplus_{k=1}^{m} \mathcal{D}_{2 k-1} \oplus \mathcal{D}_{2 k-3 / 2}\right) \oplus\left(\bigoplus_{k=1}^{m-1} \mathcal{D}_{2 k-1 / 2} \oplus \mathcal{D}_{2 k-1}\right) \\
& =\bigoplus_{k=1}^{m-1}\left(\mathcal{R}_{2 k-1} \oplus \mathcal{R}_{2 k-1 / 2}\right) \oplus \mathcal{R}_{2 m-1}
\end{aligned}
$$


By analogy with the bosonic $S p(2 m)$ case (cf. 4.8), we set (with $m$ integer)

$$
\left(\mathcal{R}_{m-1 / 2}^{\pi} \times \mathcal{R}_{m-1 / 2}^{\pi}\right)_{S}=\bigoplus_{k=1}^{m-1}\left(\mathcal{R}_{2 k-1} \oplus \mathcal{R}_{2 k-1 / 2}\right) \oplus \mathcal{R}_{2 m-1} \quad \text { with } \quad k \in \mathbb{Z}
$$

Using the equations (10.7), (10.18) and (10.22), one obtains also the useful formulae (with $k$ and $m$ integer)

$$
\left(\mathcal{R}_{m-1 / 2} \times \mathcal{R}_{m-1 / 2}\right)_{A}=\bigoplus_{k=0}^{m-1}\left(\mathcal{R}_{2 k} \oplus \mathcal{R}_{2 k+1 / 2}\right)
$$

and

$$
\left(\mathcal{R}_{m}^{\pi} \times \mathcal{R}_{m}^{\pi}\right)_{S}=\bigoplus_{k=0}^{m-1}\left(\mathcal{R}_{2 k} \oplus \mathcal{R}_{2 k+1 / 2}\right) \oplus \mathcal{R}_{2 m}
$$

The products between $\mathcal{R}_{j}$ and $\mathcal{R}_{j}^{\pi}$ representations are given by

$$
\begin{aligned}
& \mathcal{R}_{j_{1}} \times \mathcal{R}_{j_{2}}= \begin{cases}\oplus \mathcal{R}_{j_{3}} & \text { if } j_{1}+j_{2} \text { is integer } \\
\oplus \mathcal{R}_{j_{3}}^{\prime} & \text { if } j_{1}+j_{2} \text { is half-integer }\end{cases} \\
& \mathcal{R}_{j_{1}}^{\pi} \times \mathcal{R}_{j_{2}}^{\pi}= \begin{cases}\oplus \mathcal{R}_{j_{3}} & \text { if } j_{1}+j_{2} \text { is integer } \\
\oplus \mathcal{R}_{j_{3}}^{\prime} & \text { if } j_{1}+j_{2} \text { is half-integer }\end{cases} \\
& \mathcal{R}_{j_{1}} \times \mathcal{R}_{j_{2}}^{\pi}= \begin{cases}\oplus \mathcal{R}_{j_{3}}^{\prime} & \text { if } j_{1}+j_{2} \text { is integer } \\
\oplus \mathcal{R}_{j_{3}} & \text { if } j_{1}+j_{2} \text { is half-integer }\end{cases}
\end{aligned}
$$

where the representations $\mathcal{R}_{j_{3}}$ and $\mathcal{R}_{j_{3}}^{\prime}$ correspond to $W$ superfields which obey to "right" or "wrong" statistics respectively.

Finally, one has

$$
\begin{aligned}
& \left(n \mathcal{R}_{j} \times n \mathcal{R}_{j}\right)_{A}=\frac{n(n+1)}{2}\left(\mathcal{R}_{j} \times \mathcal{R}_{j}\right)_{A} \oplus \frac{n(n-1)}{2}\left(\mathcal{R}_{j} \times \mathcal{R}_{j}\right)_{S} \\
& \left(n \mathcal{R}_{j} \times n \mathcal{R}_{j}\right)_{S}=\frac{n(n+1)}{2}\left(\mathcal{R}_{j} \times \mathcal{R}_{j}\right)_{S} \oplus \frac{n(n-1)}{2}\left(\mathcal{R}_{j} \times \mathcal{R}_{j}\right)_{A}
\end{aligned}
$$

and

$$
\begin{aligned}
& \left(\left(\mathcal{R}_{j_{1}} \oplus \mathcal{R}_{j_{2}}\right) \times\left(\mathcal{R}_{j_{1}} \oplus \mathcal{R}_{j_{2}}\right)\right)_{A}=\left(\mathcal{R}_{j_{1}} \times \mathcal{R}_{j_{1}}\right)_{A} \oplus\left(\mathcal{R}_{j_{2}} \times \mathcal{R}_{j_{2}}\right)_{A} \oplus\left(\mathcal{R}_{j_{1}} \times \mathcal{R}_{j_{2}}\right) \\
& \left(\left(\mathcal{R}_{j_{1}} \oplus \mathcal{R}_{j_{2}}\right) \times\left(\mathcal{R}_{j_{1}} \oplus \mathcal{R}_{j_{2}}\right)\right)_{S}=\left(\mathcal{R}_{j_{1}} \times \mathcal{R}_{j_{1}}\right)_{A} \oplus\left(\mathcal{R}_{j_{2}} \times \mathcal{R}_{j_{2}}\right)_{S} \oplus\left(\mathcal{R}_{j_{1}} \times \mathcal{R}_{j_{2}}\right)
\end{aligned}
$$

Of course, the same formulae hold for $\mathcal{R}^{\pi}$ representations.

It remains to obtain the decompositions of the adjoint representations of the simple Lie superalgebras from the decompositions of their fundamental representations for the different possible $O S p(1 \mid 2)$ embeddings in order to classify the super-Toda theories. The following subsections are devoted to the study of the superalgebras $O S p(2 m \mid 2 n), O S p(2 m+1 \mid 2 n), O S p(2 \mid 2 n)$ and to the irregular embeddings. 


\subsubsection{The superalgebras $O S p(2 m \mid 2 n)$}

The regular SSAs of $\mathcal{G}=O S p(2 m \mid 2 n)$ (with $m \geq 2$ ) which admit a superprincipal embedding are of the type $O S p(2 k \mid 2 k), O S p(2 k+2 \mid 2 k)$ and $S l(p \pm 1 \mid p)$.

Let $\widetilde{\mathcal{G}}=O S p(2 k \mid 2 k)$ with $1 \leq k \leq \inf (m, n)$. Under the superprincipal $O S p(1 \mid 2)$ of $\widetilde{\mathcal{G}}$, the fundamental representation of $O S p(2 m \mid 2 n)$ of dimension $2 m+$ $2 n$ decomposes as follows:

$$
\underline{2 m+2 n}=\mathcal{R}_{k-1 / 2}^{\pi} \oplus(2 m-2 k+1) \mathcal{R}_{0} \oplus(2 n-2 k) \mathcal{R}_{0}^{\pi}
$$

The decomposition of the adjoint representation of $O S p(2 m \mid 2 n)$ is obtained from the decomposition of the fundamental representation by taking the antisymmetric product of the othogonal part and the symmetric product of the symplectic part; more precisely, one has

$$
\begin{aligned}
\frac{\operatorname{Ad}[O S p(2 m \mid 2 n)]}{O S p(2 k \mid 2 k)}= & \left((2 m-2 k+1) \mathcal{R}_{0}\right) \times\left.\left((2 m-2 k+1) \mathcal{R}_{0}\right)\right|_{A} \\
& \oplus\left(\mathcal{R}_{k-1 / 2}^{\pi} \oplus(2 n-2 k) \mathcal{R}_{0}^{\pi}\right) \times\left.\left(\mathcal{R}_{k-1 / 2}^{\pi} \oplus(2 n-2 k) \mathcal{R}_{0}^{\pi}\right)\right|_{S} \\
& \oplus\left((2 m-2 k+1) \mathcal{R}_{0}\right) \times\left(\mathcal{R}_{k-1 / 2}^{\pi} \oplus(2 n-2 k) \mathcal{R}_{0}^{\pi}\right)(10.31)
\end{aligned}
$$

Using the formulae (10.18) and (10.22 10.29$)$, one finds

$$
\begin{aligned}
\frac{\operatorname{Ad}[O S p(2 m \mid 2 n)]}{O S p(2 k \mid 2 k)} & =\mathcal{R}_{2 k-1} \oplus \mathcal{R}_{2 k-5 / 2} \oplus \mathcal{R}_{2 k-3} \oplus \mathcal{R}_{2 k-9 / 2} \oplus \ldots \oplus \mathcal{R}_{3 / 2} \oplus \mathcal{R}_{1} \\
& \oplus(2 m-2 k+1) \mathcal{R}_{k-1 / 2} \oplus 2(n-k) \mathcal{R}_{k-1 / 2}^{\prime} \oplus 2(2 m-2 k+1)(n-k) \mathcal{R}_{0}^{\prime} \\
& \oplus[(2 m-2 k+1)(m-k)+(2 n-2 k+1)(n-k)] \mathcal{R}_{0}
\end{aligned}
$$

Now, let $\widetilde{\mathcal{G}}=O S p(2 k+2 \mid 2 k)$. Under the superprincipal $O S p(1 \mid 2)$ of $\widetilde{\mathcal{G}}$, the fundamental representation of $O S p(2 m \mid 2 n)$ decomposes as:

$$
\underline{2 m+2 n}=\mathcal{R}_{k} \oplus(2 m-2 k-1) \mathcal{R}_{0} \oplus(2 n-2 k) \mathcal{R}_{0}^{\pi}
$$

Therefore, one has

$$
\begin{aligned}
\frac{\operatorname{Ad}[O S p(2 m \mid 2 n)]}{O S p(2 k+2 \mid 2 k)}= & \left(\mathcal{R}_{k} \oplus(2 m-2 k-1) \mathcal{R}_{0}\right) \times\left.\left(\mathcal{R}_{k} \oplus(2 m-2 k-1) \mathcal{R}_{0}\right)\right|_{A} \\
& \oplus\left((2 n-2 k) \mathcal{R}_{0}^{\pi}\right) \times\left.\left((2 n-2 k) \mathcal{R}_{0}^{\pi}\right)\right|_{S} \\
& \oplus\left(\mathcal{R}_{k} \oplus(2 m-2 k-1) \mathcal{R}_{0}\right) \times\left((2 n-2 k) \mathcal{R}_{0}^{\pi}\right)
\end{aligned}
$$


and one obtains in that case

$$
\begin{aligned}
& \frac{\mathbf{A d}[O S p(2 m \mid 2 n)]}{O S p(2 k+2 \mid 2 k)}= \mathcal{R}_{2 k-1 / 2} \oplus \mathcal{R}_{2 k-1} \oplus \mathcal{R}_{2 k-5 / 2} \oplus \mathcal{R}_{2 k-3} \oplus \ldots \oplus \mathcal{R}_{3 / 2} \oplus \mathcal{R}_{1} \\
& \oplus(2 m-2 k-1) \mathcal{R}_{k} \oplus 2(n-k) \mathcal{R}_{k}^{\prime} \oplus 2(2 m-2 k-1)(n-k) \mathcal{R}_{0}^{\prime} \\
& \oplus \quad[(2 m-2 k-1)(m-k-1)+(2 n-2 k+1)(n-k)] \mathcal{R}(10.35)
\end{aligned}
$$

Finally, let us consider the case where $\widetilde{\mathcal{G}}$ belongs to the unitary series. First, we study the case $\widetilde{\mathcal{G}}=S l(2 k+1 \mid 2 k)$ with $4 k \leq m+n-2$. The decomposition of the fundamental representation of $O S p(2 m \mid 2 n)$ under the superprincipal $O S p(1 \mid 2)$ of $\widetilde{\mathcal{G}}$ is given by

$$
\underline{2 m+2 n}=2 \mathcal{R}_{k} \oplus 2(m-2 k-1) \mathcal{R}_{0} \oplus 2(n-2 k) \mathcal{R}_{0}^{\pi}
$$

Therefore, one has

$$
\begin{aligned}
\frac{\mathbf{A d}[O S p(2 m \mid 2 n)]}{S l(2 k+1 \mid 2 k)}= & \left(2 \mathcal{R}_{k} \oplus 2(m-2 k-1) \mathcal{R}_{0}\right) \times\left.\left(2 \mathcal{R}_{k} \oplus 2(m-2 k-1) \mathcal{R}_{0}\right)\right|_{A} \\
& \oplus\left(2(n-2 k) \mathcal{R}_{0}^{\pi}\right) \times\left.\left(2(n-2 k) \mathcal{R}_{0}^{\pi}\right)\right|_{S} \\
& \oplus\left(2 \mathcal{R}_{k} \oplus 2(m-2 k-1) \mathcal{R}_{0}\right) \times\left(2(n-2 k) \mathcal{R}_{0}^{\pi}\right)
\end{aligned}
$$

One obtains here

$$
\begin{aligned}
\frac{\operatorname{Ad}[O S p(2 m \mid 2 n)]}{S l(2 k+1 \mid 2 k)}= & \mathcal{R}_{2 k} \oplus 3 \mathcal{R}_{2 k-1} \oplus \mathcal{R}_{2 k-2} \oplus \ldots \oplus \mathcal{R}_{2} \oplus 3 \mathcal{R}_{1} \oplus \mathcal{R}_{0} \\
\oplus & 3 \mathcal{R}_{2 k-1 / 2} \oplus \mathcal{R}_{2 k-3 / 2} \oplus 3 \mathcal{R}_{2 k-5 / 2} \oplus \ldots \oplus 3 \mathcal{R}_{3 / 2} \oplus \mathcal{R}_{1 / 2} \\
\oplus & 4(m-2 k-1) \mathcal{R}_{k} \oplus 4(n-2 k) \mathcal{R}_{k}^{\prime} \oplus 4(m-2 k-1)(n-2 k) \mathcal{R}_{0}^{\prime} \\
\oplus & {\left.[(2 m-4 k-3)(m-2 k-1)+(2 n-4 k+1)(n-2 k)] \mathcal{R}_{b} 0.38\right) }
\end{aligned}
$$

The other cases are similar. One finds easily the following results. If $\widetilde{\mathcal{G}}=S l(2 k-$ $1 \mid 2 k)$ with $4 k \leq m+n$, one has

$$
\underline{2 m+2 n}=2 \mathcal{R}_{k-1 / 2}^{\pi} \oplus 2(m-2 k+1) \mathcal{R}_{0} \oplus 2(n-2 k) \mathcal{R}_{0}^{\pi}
$$

and

$$
\begin{aligned}
\frac{\operatorname{Ad}[O S p(2 m \mid 2 n)]}{S l(2 k-1 \mid 2 k)}= & 3 \mathcal{R}_{2 k-1} \oplus \mathcal{R}_{2 k-2} \oplus 3 \mathcal{R}_{2 k-3} \oplus \ldots \oplus \mathcal{R}_{2} \oplus 3 \mathcal{R}_{1} \oplus \mathcal{R}_{0} \\
\oplus & \mathcal{R}_{2 k-3 / 2} \oplus 3 \mathcal{R}_{2 k-5 / 2} \oplus \mathcal{R}_{2 k-7 / 2} \oplus \ldots \oplus 3 \mathcal{R}_{3 / 2} \oplus \mathcal{R}_{1 / 2} \\
\oplus & 4(m-2 k+1) \mathcal{R}_{k-1 / 2} \oplus 4(n-2 k) \mathcal{R}_{k-1 / 2}^{\prime} \oplus 4(m-2 k+1)(n-2 k) \mathcal{R}_{0}^{\prime} \\
\oplus & {[(2 m-4 k+1)(m-2 k+1)+(2 n-4 k+1)(n-2 k)] \mathcal{R}_{0} \quad(10.40) }
\end{aligned}
$$


If $\widetilde{\mathcal{G}}=S l(2 k \mid 2 k+1)$, one has

$$
\underline{2 m+2 n}=2 \mathcal{R}_{k}^{\pi} \oplus 2(m-2 k) \mathcal{R}_{0} \oplus 2(n-2 k-1) \mathcal{R}_{0}^{\pi}
$$

and

$$
\begin{aligned}
\frac{\mathbf{A d}[O S p(2 m \mid 2 n)]}{S l(2 k \mid 2 k+1)}= & 3 \mathcal{R}_{2 k} \oplus \mathcal{R}_{2 k-1} \oplus 3 \mathcal{R}_{2 k-2} \oplus \ldots \oplus 3 \mathcal{R}_{2} \oplus \mathcal{R}_{1} \oplus 3 \mathcal{R}_{0} \\
\oplus & \mathcal{R}_{2 k-1 / 2} \oplus 3 \mathcal{R}_{2 k-3 / 2} \oplus \mathcal{R}_{2 k-5 / 2} \oplus \ldots \oplus \mathcal{R}_{3 / 2} \oplus 3 \mathcal{R}_{1 / 2} \\
\oplus & 4(m-2 k) \mathcal{R}_{k}^{\prime} \oplus 4(n-2 k-1) \mathcal{R}_{k} \oplus 4(m-2 k)(n-2 k-1) \mathcal{R}_{0}^{\prime} \\
\oplus & {\left.[(2 m-4 k-1)(m-2 k)+(2 n-4 k-1)(n-2 k-1)] \mathcal{R}_{b} 0.42\right) }
\end{aligned}
$$

Finally, if $\widetilde{\mathcal{G}}=S l(2 k \mid 2 k-1)$, one has

$$
\underline{2 m+2 n}=2 \mathcal{R}_{k-1 / 2} \oplus 2(m-2 k) \mathcal{R}_{0} \oplus 2(n-2 k+1) \mathcal{R}_{0}^{\pi}
$$

and

$$
\begin{aligned}
\frac{\operatorname{Ad}[O S p(2 m \mid 2 n)]}{S l(2 k \mid 2 k-1)}= & \mathcal{R}_{2 k-1} \oplus 3 \mathcal{R}_{2 k-2} \oplus \mathcal{R}_{2 k-3} \oplus \ldots \oplus 3 \mathcal{R}_{2} \oplus \mathcal{R}_{1} \oplus 3 \mathcal{R}_{0} \\
\oplus & 3 \mathcal{R}_{2 k-3 / 2} \oplus \mathcal{R}_{2 k-5 / 2} \oplus 3 \mathcal{R}_{2 k-7 / 2} \oplus \ldots \oplus \mathcal{R}_{3 / 2} \oplus 3 \mathcal{R}_{1 / 2} \\
\oplus & 4(m-2 k) \mathcal{R}_{k-1 / 2}^{\prime} \oplus 4(n-2 k+1) \mathcal{R}_{k-1 / 2} \oplus 4(m-2 k)(n-2 k+1) \mathcal{R}_{0}^{\prime} \\
\oplus & {[(2 m-4 k-1)(m-2 k)+(2 n-4 k+3)(n-2 k+1)] \mathcal{R}_{0} \quad(10.44) }
\end{aligned}
$$

\subsubsection{The superalgebras $O S p(2 m+1 \mid 2 n)$}

The regular SSAs of $\mathcal{G}=O S p(2 m+1 \mid 2 n)$ which admit a superprincipal embedding are of the type $O S p(2 k \mid 2 k), O S p(2 k+2 \mid 2 k), O S p(2 k \pm 1 \mid 2 k)$ and $S l(p \pm 1 \mid p)$.

Let $\widetilde{\mathcal{G}}=O S p(2 k \mid 2 k)$. Under the superprincipal $O S p(1 \mid 2)$ of $\widetilde{\mathcal{G}}$, the fundamental representation of $O S p(2 m+1 \mid 2 n)$, of dimension $2 m+2 n+1$, decomposes as follows:

$$
\underline{2 m+2 n+1}=\mathcal{R}_{k-1 / 2}^{\pi} \oplus(2 n-2 k) \mathcal{R}_{0}^{\pi} \oplus(2 m-2 k+2) \mathcal{R}_{0}
$$

The decomposition of the adjoint representation is then

$$
\begin{aligned}
\frac{\mathbf{A d}[O S p(2 m+1 \mid 2 n)]}{O S p(2 k \mid 2 k)}= & \left((2 m-2 k+2) \mathcal{R}_{0}\right) \times\left.\left((2 m-2 k+2) \mathcal{R}_{0}\right)\right|_{A} \\
& \oplus\left(\mathcal{R}_{k-1 / 2}^{\pi} \oplus(2 n-2 k) \mathcal{R}_{0}^{\pi}\right) \times\left.\left(\mathcal{R}_{k-1 / 2}^{\pi} \oplus(2 n-2 k) \mathcal{R}_{0}^{\pi}\right)\right|_{S} \\
& \oplus\left((2 m-2 k+2) \mathcal{R}_{0}\right) \times\left(\mathcal{R}_{k-1 / 2}^{\pi} \oplus(2 n-2 k) \mathcal{R}_{0}^{\pi}\right)(10.46)
\end{aligned}
$$


i.e.

$$
\begin{aligned}
& \frac{\operatorname{Ad}[O S p(2 m+1 \mid 2 n)]}{O S p(2 k \mid 2 k)}=\mathcal{R}_{2 k-1} \oplus \mathcal{R}_{2 k-5 / 2} \oplus \mathcal{R}_{2 k-3} \oplus \mathcal{R}_{2 k-9 / 2} \oplus \ldots \oplus \mathcal{R}_{3 / 2} \oplus \mathcal{R}_{1} \\
& \oplus 2(m-k+1) \mathcal{R}_{k-1 / 2} \oplus 2(n-k) \mathcal{R}_{k-1 / 2}^{\prime} \oplus 4(m-k+1)(n-k) \mathcal{R}_{0}^{\prime} \\
& \oplus[(2 m-2 k+1)(m-k+1)+(2 n-2 k+1)(n-k)] \mathcal{R}_{0} \quad(10.47)
\end{aligned}
$$

Now, let $\widetilde{\mathcal{G}}=\operatorname{OSp}(2 k+2 \mid 2 k)$. Under the superprincipal $\operatorname{OSp}(1 \mid 2)$ of $\widetilde{\mathcal{G}}$, the fundamental representation of $O S p(2 m+1 \mid 2 n)$ decomposes as:

$$
\underline{2 m+2 n+1}=\mathcal{R}_{k} \oplus(2 m-2 k) \mathcal{R}_{0} \oplus(2 n-2 k) \mathcal{R}_{0}^{\pi}
$$

Then one obtains

$$
\begin{aligned}
\frac{\operatorname{Ad}[O S p(2 m+1 \mid 2 n)]}{O S p(2 k+2 \mid 2 k)}= & \left(\mathcal{R}_{k} \oplus(2 m-2 k) \mathcal{R}_{0}\right) \times\left.\left(\mathcal{R}_{k} \oplus(2 m-2 k) \mathcal{R}_{0}\right)\right|_{A} \\
& \oplus\left((2 n-2 k) \mathcal{R}_{0}^{\pi}\right) \times\left.\left((2 n-2 k) \mathcal{R}_{0}^{\pi}\right)\right|_{S} \\
& \oplus\left(\mathcal{R}_{k} \oplus(2 m-2 k) \mathcal{R}_{0}\right) \times\left((2 n-2 k) \mathcal{R}_{0}^{\pi}\right)
\end{aligned}
$$

i.e.

$$
\begin{aligned}
\frac{\operatorname{Ad}[O S p(2 m+1 \mid 2 n)]}{O S p(2 k+2 \mid 2 k)}= & \mathcal{R}_{2 k-1 / 2} \oplus \mathcal{R}_{2 k-1} \oplus \mathcal{R}_{2 k-5 / 2} \oplus \mathcal{R}_{2 k-3} \oplus \ldots \oplus \mathcal{R}_{3 / 2} \oplus \mathcal{R}_{1} \\
\oplus & 2(n-k) \mathcal{R}_{k}^{\prime} \oplus 2(m-k) \mathcal{R}_{k} \oplus 4(m-k)(n-k) \mathcal{R}_{0}^{\prime} \\
\oplus & {[(2 m-2 k-1)(m-k)+(2 n-2 k+1)(n-k)]\left(\mathbb{R}_{0.50)}\right.}
\end{aligned}
$$

Finally, let $\widetilde{\mathcal{G}}=O S p(2 k-1 \mid 2 k)$. Under the superprincipal $O S p(1 \mid 2)$ of $\widetilde{\mathcal{G}}$, the fundamental representation of $O S p(2 m+1 \mid 2 n)$ decomposes as

$$
\underline{2 m+2 n+1}=\mathcal{R}_{k-1 / 2}^{\pi} \oplus(2 n-2 k) \mathcal{R}_{0}^{\pi} \oplus(2 m-2 k+2) \mathcal{R}_{0}
$$

which is the same decomposition as the case $\widetilde{\mathcal{G}}=O S p(2 k \mid 2 k)$. Therefore, the two SSAs $O S p(2 k \mid 2 k)$ and $O S p(2 k-1 \mid 2 k$ ) (when both can be embedded in $\mathcal{G}$ ) lead to the same decomposition of the adjoint representation of $\mathcal{G}$ and consequently to the same theory. On the same lines, one finds that the two $\operatorname{SSAs} O S p(2 k+2 \mid 2 k)$ and $O S p(2 k+1 \mid 2 k)$ lead to the same theory.

The last case is $\widetilde{\mathcal{G}}=S l(p \pm 1 \mid p)$. We leave the different decompositions to the reader. The results are summarized in the table of Section 10.3 . 


\subsubsection{The irregular embeddings}

We will study now the irregular embeddings, which are present in $O S p(2 n \pm$ $2 \mid 2 n)$ and $O S p(2 n \mid 2 n)$.

Consider first the superalgebra $\mathcal{G}=O S p(2 n+2 \mid 2 n)$ and take the $O S p(1 \mid 2)$ SSA of $\mathcal{G}$ such that the minimal including SSA in $\mathcal{G}$ (which is now singular) is $\widetilde{\mathcal{G}}=O S p(2 k+1 \mid 2 k) \oplus O S p(2 n-2 k+1 \mid 2 n-2 k)$ and $1 \leq k \leq\left[\frac{n-1}{2}\right]$. Under the superprincipal $O S p(1 \mid 2)$ of $\widetilde{\mathcal{G}}$, the fundamental representation of $\mathcal{G}$, of dimension $4 n+2$, decomposes as

$$
\underline{4 n+2}=\mathcal{R}_{k} \oplus \mathcal{R}_{n-k}
$$

and we get for the $O S p(2 n+2 \mid 2 n)$ adjoint representation

$\frac{\operatorname{Ad}[O S p(2 n+2 \mid 2 n)]}{O S p(2 k+1 \mid 2 k) \oplus O S p(2 n-2 k+1 \mid 2 n-2 k)}=\left(\mathcal{R}_{k} \oplus \mathcal{R}_{n-k}\right) \times\left.\left(\mathcal{R}_{k} \oplus \mathcal{R}_{n-k}\right)\right|_{A}$

which leads to the following decomposition:

$$
\begin{aligned}
& \frac{\operatorname{Ad}[O S p(2 n+2 \mid 2 n)]}{O S p(2 k+1 \mid 2 k) \oplus O S p(2 n-2 k+1 \mid 2 n-2 k)}=\mathcal{R}_{2 n-2 k-1} \oplus \mathcal{R}_{2 n-2 k-3} \oplus \ldots \oplus \mathcal{R}_{1} \\
& \oplus \mathcal{R}_{2 n-2 k-1 / 2} \oplus \mathcal{R}_{2 n-2 k-3 / 2} \oplus \ldots \oplus \mathcal{R}_{3 / 2} \oplus \mathcal{R}_{2 k-1} \oplus \mathcal{R}_{2 k-3} \oplus \ldots \oplus \mathcal{R}_{1} \\
& \oplus \mathcal{R}_{2 k-1 / 2} \oplus \mathcal{R}_{2 k-3 / 2} \oplus \ldots \oplus \mathcal{R}_{3 / 2} \oplus \mathcal{R}_{n} \oplus \mathcal{R}_{n-1} \oplus \ldots \oplus \mathcal{R}_{n-2 k} \\
& \oplus \mathcal{R}_{n-1 / 2} \oplus \mathcal{R}_{n-3 / 2} \oplus \ldots \oplus \mathcal{R}_{n-2 k+1 / 2}
\end{aligned}
$$

Consider then the superalgebra $\mathcal{G}=O S p(2 n-2 \mid 2 n)$ with $\widetilde{\mathcal{G}}=O S p(2 k-$ $1 \mid 2 k) \oplus O S p(2 n-2 k-1 \mid 2 n-2 k)$ and $1 \leq k \leq\left[\frac{n-3}{2}\right]$. The fundamental representation of $\mathcal{G}$, of dimension $4 n-2$, decomposes under the superprincipal $\operatorname{OSp}(1 \mid 2)$ of $\widetilde{\mathcal{G}}$ as

$$
\underline{4 n-2}=\mathcal{R}_{k-1 / 2}^{\pi} \oplus \mathcal{R}_{n-k-1 / 2}^{\pi}
$$

The adjoint representation of $O S p(2 n-2 \mid 2 n)$ is given by

$$
\frac{\operatorname{Ad}[O S p(2 n-2 \mid 2 n)]}{O S p(2 k-1 \mid 2 k) \oplus O S p(2 n-2 k-1 \mid 2 n-2 k)}=\left(\mathcal{R}_{k-1 / 2}^{\pi} \oplus \mathcal{R}_{n-k-1 / 2}^{\pi}\right) \times\left.\left(\mathcal{R}_{k-1 / 2}^{\pi} \oplus \mathcal{R}_{n-k-1 / 2}^{\pi}\right)\right|_{S}
$$

i.e.

$$
\begin{aligned}
& \frac{\operatorname{Ad}[O S p(2 n-2 \mid 2 n)]}{O S p(2 k-1 \mid 2 k) \oplus O S p(2 n-2 k-1 \mid 2 n-2 k)}=\mathcal{R}_{2 n-2 k-1} \oplus \mathcal{R}_{2 n-2 k-3} \oplus \ldots \oplus \mathcal{R}_{1} \\
& \oplus \mathcal{R}_{2 n-2 k-5 / 2} \oplus \mathcal{R}_{2 n-2 k-7 / 2} \oplus \ldots \oplus \mathcal{R}_{3 / 2} \oplus \mathcal{R}_{2 k-1} \oplus \mathcal{R}_{2 k-3} \oplus \ldots \oplus \mathcal{R}_{1} \\
& \oplus \mathcal{R}_{2 k-5 / 2} \oplus \mathcal{R}_{2 k-7 / 2} \oplus \ldots \oplus \mathcal{R}_{3 / 2} \oplus \mathcal{R}_{n-1} \oplus \mathcal{R}_{n-2} \oplus \ldots \oplus \mathcal{R}_{n-2 k} \\
& \oplus \mathcal{R}_{n-3 / 2} \oplus \mathcal{R}_{n-5 / 2} \oplus \ldots \oplus \mathcal{R}_{n-2 k+1 / 2}
\end{aligned}
$$


Consider finally the superalgebra $\mathcal{G}=O S p(2 n \mid 2 n)$ with $\widetilde{\mathcal{G}}=O S p(2 k+1 \mid 2 k) \oplus$ $O S p(2 n-2 k-1 \mid 2 n-2 k)$ and $1 \leq k \leq\left[\frac{n-1}{2}\right]$. Under the superprincipal $\operatorname{OSp}(1 \mid 2)$ of $\widetilde{\mathcal{G}}$, the fundamental representation of $\mathcal{G}$, of dimension $4 n$, decomposes as

$$
\underline{4 n}=\mathcal{R}_{k} \oplus \mathcal{R}_{n-k-1 / 2}^{\pi}
$$

and we get for the $O S p(2 n \mid 2 n)$ adjoint representation

$$
\begin{aligned}
\frac{\operatorname{Ad}[O S p(2 n \mid 2 n)]}{O S p(2 k+1 \mid 2 k) \oplus O S p(2 n-2 k-1 \mid 2 n-2 k)}= & \left(\mathcal{R}_{k} \times \mathcal{R}_{k}\right)_{A} \oplus\left(\mathcal{R}_{n-k-1 / 2}^{\pi} \times \mathcal{R}_{n-k-1 / 2}^{\pi}\right)_{S} \\
& \oplus\left(\mathcal{R}_{k} \times \mathcal{R}_{n-k-1 / 2}^{\pi}\right)
\end{aligned}
$$

which leads to

$$
\begin{aligned}
& \frac{\operatorname{Ad}[O S p(2 n \mid 2 n)]}{O S p(2 k+1 \mid 2 k) \oplus O S p(2 n-2 k-1 \mid 2 n-2 k)}=\mathcal{R}_{2 n-2 k-1} \oplus \mathcal{R}_{2 n-2 k-3} \oplus \ldots \oplus \mathcal{R}_{1} \\
& \oplus \mathcal{R}_{2 n-2 k-5 / 2} \oplus \mathcal{R}_{2 n-2 k-7 / 2} \oplus \ldots \oplus \mathcal{R}_{3 / 2} \oplus \mathcal{R}_{2 k-1} \oplus \mathcal{R}_{2 k-3} \oplus \ldots \oplus \mathcal{R}_{1} \\
& \oplus \mathcal{R}_{2 k-1 / 2} \oplus \mathcal{R}_{2 k-3 / 2} \oplus \ldots \oplus \mathcal{R}_{3 / 2} \oplus \mathcal{R}_{n-1} \oplus \mathcal{R}_{n-2} \oplus \ldots \oplus \mathcal{R}_{n-2 k} \\
& \oplus \mathcal{R}_{n-1 / 2} \oplus \mathcal{R}_{n-3 / 2} \oplus \ldots \oplus \mathcal{R}_{n-2 k-1 / 2}
\end{aligned}
$$

If $\widetilde{\mathcal{G}}=O S p(2 k-1 \mid 2 k) \oplus O S p(2 n-2 k+1 \mid 2 n-2 k)$ with $1 \leq k \leq\left[\frac{n-2}{2}\right]$, the fundamental representation of $\mathcal{G}$, of dimension $4 n$, decomposes under the superprincipal $O S p(1 \mid 2)$ of $\widetilde{\mathcal{G}}$ as

$$
\underline{4 n}=\mathcal{R}_{n-k} \oplus \mathcal{R}_{k-1 / 2}^{\pi}
$$

and we have the following decomposition of the adjoint representation of $O S p(2 n \mid 2 n)$ :

$$
\begin{aligned}
& \frac{\operatorname{Ad}[O S p(2 n \mid 2 n)]}{O S p(2 k-1 \mid 2 k) \oplus O S p(2 n-2 k+1 \mid 2 n-2 k)}=\mathcal{R}_{2 n-2 k-1} \oplus \mathcal{R}_{2 n-2 k-3} \oplus \ldots \oplus \mathcal{R}_{1} \\
& \oplus \mathcal{R}_{2 n-2 k-1 / 2} \oplus \mathcal{R}_{2 n-2 k-3 / 2} \oplus \ldots \oplus \mathcal{R}_{3 / 2} \oplus \mathcal{R}_{2 k-1} \oplus \mathcal{R}_{2 k-3} \oplus \ldots \oplus \mathcal{R}_{1} \\
& \oplus \mathcal{R}_{2 k-5 / 2} \oplus \mathcal{R}_{2 k-7 / 2} \oplus \ldots \oplus \mathcal{R}_{3 / 2} \oplus \mathcal{R}_{n-1} \oplus \mathcal{R}_{n-2} \oplus \ldots \oplus \mathcal{R}_{n-2 k+1} \\
& \oplus \mathcal{R}_{n-1 / 2} \oplus \mathcal{R}_{n-3 / 2} \oplus \ldots \oplus \mathcal{R}_{n-2 k+1 / 2}
\end{aligned}
$$

\subsubsection{The superalgebras $O S p(2 \mid 2 n)$}

The superalgebra $O S p(2 \mid 2 n)$ requires a special attention. Actually, the regular SSAs of $\mathcal{G}=O S p(2 \mid 2 n)$ which admit a superprincipal embedding are only $O S p(2 \mid 2)$ and $S l(1 \mid 2)$.

Let $\widetilde{\mathcal{G}}=O S p(2 \mid 2)$. Under the superprincipal $O S p(1 \mid 2)$ of $\widetilde{\mathcal{G}}$, the fundamental representation of $\operatorname{OSp}(2 \mid 2 n)$, of dimension $2 n+2$, decomposes as follows:

$$
\underline{2 n+2}=\mathcal{R}_{1 / 2}^{\pi} \oplus \mathcal{R}_{0} \oplus(2 n-2) \mathcal{R}_{0}^{\pi}
$$


Therefore, the decomposition of the adjoint representation of $O S p(2 \mid 2 n)$ under the superprincipal $O S p(1 \mid 2)$ of $O S p(2 \mid 2) \subset O S p(2 \mid 2 n)$ is

$$
\frac{\operatorname{Ad}[O S p(2 \mid 2 n)]}{O S p(2 \mid 2)}=\mathcal{R}_{1} \oplus \mathcal{R}_{1 / 2} \oplus(2 n-2) \mathcal{R}_{1 / 2}^{\prime} \oplus(2 n-1)(n-1) \mathcal{R}_{0} \oplus(2 n-2) \mathbb{R}(6.64)
$$

Now, let $\widetilde{\mathcal{G}}=S l(1 \mid 2)$. Under the superprincipal $O S p(1 \mid 2)$ of $\widetilde{\mathcal{G}}$, the fundamental representation of $O S p(2 \mid 2 n)$ decomposes as:

$$
\underline{2 n+2}=2 \mathcal{R}_{1 / 2}^{\pi} \oplus(2 n-4) \mathcal{R}_{0}^{\pi}
$$

In that case, the decomposition of the adjoint representation is

$$
\left.\frac{\operatorname{Ad}[O S p(2 \mid 2 n)]}{\operatorname{Sl}(1 \mid 2)}=3 \mathcal{R}_{1} \oplus \mathcal{R}_{1 / 2} \oplus(4 n-8) \mathcal{R}_{1 / 2}^{\prime} \oplus[(2 n-3)(n-2)+1] \mathcal{R} 610.66\right)
$$

\subsection{Summary of the results}

The previous results can be easily extended to the case of sums of simple Lie SSAs. The decomposition of the fundamental representation is obtained by taking the corresponding $O S p(1 \mid 2)$ representation for each factor of the sum, which can be read in the following tableau. Then, starting from a decomposition of the fundamental representation of the form

$$
\mathbf{F}=\left(\bigoplus_{i} \mathcal{R}_{i}\right) \oplus\left(\bigoplus_{j} \mathcal{R}_{j}^{\pi}\right)
$$

the decomposition of the adjoint is given, in the orthosymplectic series, by

$$
\mathbf{A d}=\left(\bigoplus_{i} \mathcal{R}_{i}\right) \times\left.\left(\bigoplus_{i} \mathcal{R}_{i}\right)\right|_{A} \oplus\left(\bigoplus_{j} \mathcal{R}_{j}^{\pi}\right) \times\left.\left(\bigoplus_{j} \mathcal{R}_{j}^{\pi}\right)\right|_{S} \oplus\left(\bigoplus_{i} \mathcal{R}_{i}\right) \times\left(\underset{j}{\bigoplus_{j}} \mathcal{R}_{j}^{\pi}\right)
$$

and in the unitary series, by

$$
\begin{aligned}
& \mathbf{A d}=\left(\bigoplus_{i} \mathcal{R}_{i} \bigoplus_{j} \mathcal{R}_{j}^{\pi}\right) \times\left(\underset{i}{\bigoplus_{i}} \mathcal{R}_{i} \bigoplus_{j} \mathcal{R}_{j}^{\pi}\right)-\mathcal{R}_{0} \quad \text { for } \quad S L(m \mid n) m(096) \\
& \mathbf{A d}=\left(\bigoplus_{i} \mathcal{R}_{i} \bigoplus_{j} \mathcal{R}_{j}^{\pi}\right) \times\left(\bigoplus_{i} \mathcal{R}_{i} \bigoplus_{j} \mathcal{R}_{j}^{\pi}\right)-2 \mathcal{R}_{0} \quad \text { for } \quad \operatorname{PSl}(m \mid m)(10.70)
\end{aligned}
$$

For explicit formulae, one has to apply the product rules given in (10.18) and (10.22-10.29). 


\begin{tabular}{|c|c|c|}
\hline $\mathcal{G}$ & $\widetilde{\mathcal{G}}$ & Fund. Rep. of $\mathcal{G}$ \\
\hline \multirow[t]{2}{*}{$S l(m \mid n)$} & $S l(p+1 \mid p)$ & $\mathcal{R}_{p / 2} \oplus(m-p-1) \mathcal{R}_{0} \oplus(n-p) \mathcal{R}_{0}^{\pi}$ \\
\hline & $S l(p \mid p+1)$ & $\mathcal{R}_{p / 2}^{\pi} \oplus(m-p) \mathcal{R}_{0} \oplus(n-p-1) \mathcal{R}_{0}^{\pi}$ \\
\hline \multirow[t]{4}{*}{$O S p(2 m \mid 2 n)$} & $O S p(2 k \mid 2 k)$ & $\begin{array}{l}\mathcal{R}_{k-1 / 2}^{\pi} \oplus(2 n-2 k) \mathcal{R}_{0}^{\pi} \\
\quad \oplus(2 m-2 k+1) \mathcal{R}_{0}\end{array}$ \\
\hline & $O S p(2 k+2 \mid 2 k)$ & $\begin{array}{c}\mathcal{R}_{k} \oplus(2 m-2 k-1) \mathcal{R}_{0} \\
\oplus(2 n-2 k) \mathcal{R}_{0}^{\pi}\end{array}$ \\
\hline & $S l(p+1 \mid p)$ & $\begin{array}{c}2 \mathcal{R}_{p / 2} \oplus 2(m-p-1) \mathcal{R}_{0} \\
\oplus 2(n-p) \mathcal{R}_{0}^{\pi}\end{array}$ \\
\hline & $S l(p \mid p+1)$ & $\begin{array}{c}2 \mathcal{R}_{p / 2}^{\pi} \oplus 2(n-p-1) \mathcal{R}_{0}^{\pi} \\
\oplus 2(m-p) \mathcal{R}_{0}\end{array}$ \\
\hline \multirow[t]{4}{*}{$O S p(2 m+1 \mid 2 n)$} & $\begin{array}{c}O S p(2 k \mid 2 k) \\
O S p(2 k-1 \mid 2 k)\end{array}$ & $\begin{array}{l}\mathcal{R}_{k-1 / 2}^{\pi} \oplus(2 n-2 k) \mathcal{R}_{0}^{\pi} \\
\quad \oplus(2 m-2 k+2) \mathcal{R}_{0}\end{array}$ \\
\hline & $\begin{array}{l}O S p(2 k+2 \mid 2 k) \\
O S p(2 k+1 \mid 2 k)\end{array}$ & $\begin{array}{l}\mathcal{R}_{k} \oplus(2 m-2 k) \mathcal{R}_{0} \\
\oplus(2 n-2 k) \mathcal{R}_{0}^{\pi}\end{array}$ \\
\hline & $S l(p+1 \mid p)$ & $\begin{array}{l}2 \mathcal{R}_{p / 2} \oplus 2(m-p-1) \mathcal{R}_{0} \\
\quad \oplus \mathcal{R}_{0} \oplus 2(n-p) \mathcal{R}_{0}^{\pi}\end{array}$ \\
\hline & $S l(p \mid p+1)$ & $\begin{array}{l}2 \mathcal{R}_{p / 2}^{\pi} \oplus 2(n-p-1) \mathcal{R}_{0}^{\pi} \\
\quad \oplus \mathcal{R}_{0} \oplus 2(m-p) \mathcal{R}_{0}\end{array}$ \\
\hline \multirow[t]{2}{*}{$O S p(2 \mid 2 n)$} & $O S p(2 \mid 2)$ & $\mathcal{R}_{1 / 2}^{\pi} \oplus \mathcal{R}_{0} \oplus(2 n-2) \mathcal{R}_{0}^{\pi}$ \\
\hline & $S l(1 \mid 2)$ & $2 \mathcal{R}_{1 / 2}^{\pi} \oplus(2 n-4) \mathcal{R}_{0}^{\pi}$ \\
\hline
\end{tabular}




\begin{tabular}{|c|c|c|}
\hline $\mathcal{G}$ & $\widetilde{\mathcal{G}}$ & Fund. Rep. of $\mathcal{G}$ \\
\hline$O S p(2 n+2 \mid 2 n)$ & $\begin{array}{c}O S p(2 k+1 \mid 2 k) \oplus \\
O S p(2 n-2 k+1 \mid 2 n-2 k)\end{array}$ & $\mathcal{R}_{k} \oplus \mathcal{R}_{n-k}$ \\
\hline$O S p(2 n-2 \mid 2 n)$ & $\begin{array}{c}O S p(2 k-1 \mid 2 k) \oplus \\
O S p(2 n-2 k-1 \mid 2 n-2 k)\end{array}$ & $\mathcal{R}_{k-1 / 2}^{\pi} \oplus \mathcal{R}_{n-k-1 / 2}^{\pi}$ \\
\hline \multirow[t]{2}{*}{$O S p(2 n \mid 2 n)$} & $\begin{array}{c}O S p(2 k+1 \mid 2 k) \oplus \\
O S p(2 n-2 k-1 \mid 2 n-2 k)\end{array}$ & $\mathcal{R}_{k} \oplus \mathcal{R}_{n-k-1 / 2}^{\pi}$ \\
\hline & $\begin{array}{c}O S p(2 k-1 \mid 2 k) \oplus \\
O S p(2 n-2 k+1 \mid 2 n-2 k)\end{array}$ & $\mathcal{R}_{n-k} \oplus \mathcal{R}_{k-1 / 2}^{\pi}$ \\
\hline
\end{tabular}

\subsection{The exceptional superalgebra G(3)}

The superalgebra $G(3)$ has dimension 31 and rank 3, with $\mathcal{G}_{B}=G_{2} \oplus S l(2)$ as bosonic part and the representation $(\underline{7}, \underline{2})$ of $\mathcal{G}_{B}$ as fermionic part. The Dynkin diagrams of $G(3)$ are
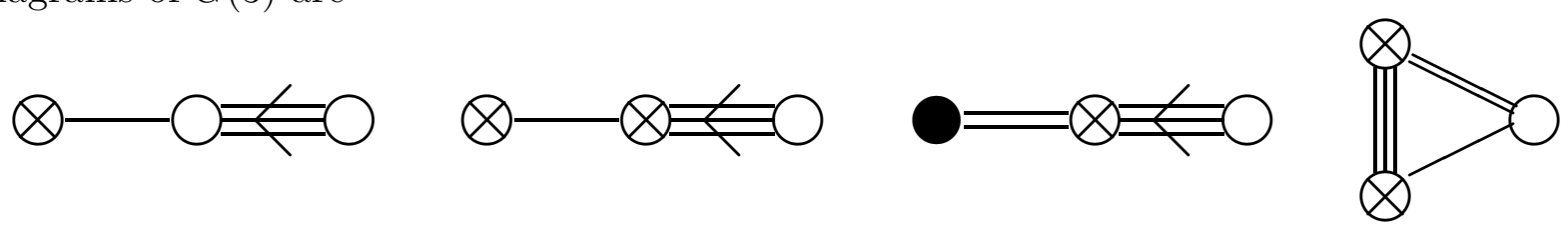

leading to the following regular sub(super)algebras:

$$
\begin{aligned}
& G_{2} \oplus A_{1}, G_{2}, A_{2}, A_{1} \\
& B(1,1) \oplus A_{1}, B(1,1), C(2), B(0,1), A_{2} \oplus B(0,1) \\
& A(0,2), A(0,1), A(1,0), D(2,1 ; 3), G(3)
\end{aligned}
$$

Only the superalgebras $B(0,1), C(2), B(1,1), A(0,1), A(1,0)$ and $D(2,1 ; 3)$ admit a superprincipal embedding. As an example, we will treat the case of $B(1,1) \equiv O S p(3 \mid 2)$. From the results of Section 5.4, the bosonic part $G_{2} \oplus S l(2)$ decomposes under the principal $S l(2)$ of $S O(3) \oplus S l(2)$ as

$$
\operatorname{Ad}\left[G_{2} \oplus S l(2)\right]=\mathcal{D}_{3 / 2} \oplus \overline{\mathcal{D}}_{3 / 2} \oplus 2 \mathcal{D}_{1} \oplus 3 \mathcal{D}_{0}
$$

and the fermionic part $(\underline{7}, \underline{2})$ as

$$
(\underline{7}, \underline{2})=\mathcal{D}_{3 / 2} \oplus 2 \mathcal{D}_{1} \oplus \mathcal{D}_{1 / 2} \oplus 2 \mathcal{D}_{0}
$$

Putting together the $S l(2)$ representations into $O S p(1 \mid 2)$ ones, one obtains the following decomposition under the superprincipal $\operatorname{OSp}(1 \mid 2)$ of $O S p(3 \mid 2) \subset G(3)$ :

$$
\frac{\operatorname{Ad}[G(3)]}{O S p(3 \mid 2)}=\mathcal{R}_{3 / 2} \oplus 2 \mathcal{R}_{3 / 2}^{\prime} \oplus \mathcal{R}_{1} \oplus 3 \mathcal{R}_{0} \oplus 2 \mathcal{R}_{0}^{\prime}
$$


The other cases are similar and are summarized in the table 15 .

\subsection{The exceptional superalgebra $\mathrm{F}(4)$}

The superalgebra $F(4)$ has dimension 40 and rank 4 , with $\mathcal{G}_{B}=S l(2) \oplus O(7)$ as bosonic part and the representation $(\underline{2}, \underline{8})$ of $\mathcal{G}_{B}$ as fermionic part. Its Dynkin diagrams are:
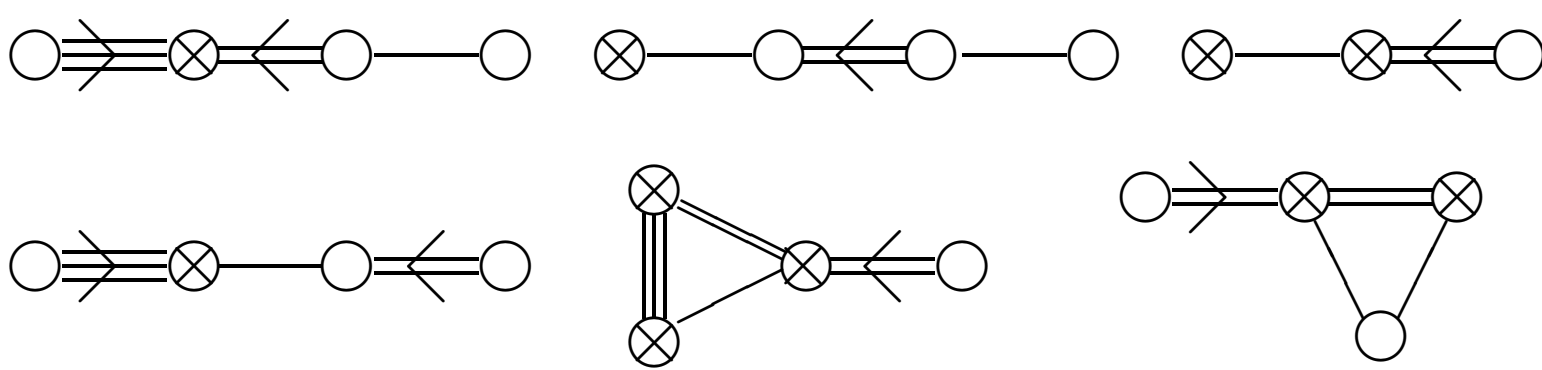

The SSAs of $F(4)$ which admit a superprincipal embedding are $A(0,1), A(1,0)$, $C(2)$ and $D(2,1 ; 2)$ (the extended Dynkin diagrams of $F(4)$ can be found in 18]). As an example, we will treat the case of $C(2) \equiv O S p(2 \mid 2)$. The bosonic part $S l(2) \oplus O(7)$ decomposes then as

$$
\operatorname{Ad}[S l(2) \oplus O(7)]=5 \mathcal{D}_{1} \oplus 9 \mathcal{D}_{0}
$$

and the fermionic part $(\underline{2}, \underline{8})$ as

$$
(\underline{2}, \underline{8})=8 \mathcal{D}_{1 / 2}
$$

Putting together the $S l(2)$ representations into $O S p(1 \mid 2)$ ones, one obtains the following decomposition under the superprincipal $O S p(1 \mid 2)$ of $O S p(2 \mid 2) \subset F(4)$ :

$$
\frac{\operatorname{Ad}[F(4)]}{O S p(2 \mid 2)}=5 \mathcal{R}_{1} \oplus 3 \mathcal{R}_{1 / 2} \oplus 6 \mathcal{R}_{0}
$$

The other cases are analogous and are summarized in the table 17 .

\section{$11 O S p(1 \mid 2) \oplus U(1)$ decompositions of simple Lie superalgebras}

\subsection{Introduction of the $U(1)$}

Now, we are in position to introduce the $U(1)$ factor. In the case of the unitary superalgebras, since the formulae for $S l(p+1 \mid p)$ are completely analogous to those of $S l(n)$ (the $\mathcal{R}_{j}$ representations replacing the $\mathcal{D}_{j}$ ones), one can write the following statement. 
A decomposition of the fundamental representation $\mathbf{F}$ of $\mathcal{G}=S l(m \mid n)$ under the superprincipal $O S p(1 \mid 2)$ of $\widetilde{\mathcal{G}} \subset \mathcal{G}$ being given,

$$
\mathbf{F}=\left(\bigoplus_{i} n_{i} \mathcal{R}_{i}\right) \oplus\left(\bigoplus_{j} n_{j} \mathcal{R}_{j}^{\pi}\right)
$$

the corresponding decomposition under $O S p(1 \mid 2) \oplus U(1)$ has the form

$$
\mathbf{F}=\left(\bigoplus_{i} n_{i} \mathcal{R}_{i}\left(y_{i}\right)\right) \oplus\left(\bigoplus_{j} n_{j} \mathcal{R}_{j}^{\pi}\left(y_{j}\right)\right)
$$

identical representations (i.e. labelled by the same index $i$ or $j$ ) having the same value of $y$. Moreover, one has to impose the supertraceless condition

$$
\sum_{i} n_{i} y_{i}-\sum_{j} n_{j} y_{j}=0
$$

Then the decomposition of the adjoint is given by

$$
\mathbf{A d}=\left(\bigoplus_{i} n_{i} \mathcal{R}_{i}\left(y_{i}\right) \bigoplus_{j} n_{j} \mathcal{R}_{j}^{\pi}\left(y_{j}\right)\right) \times\left(\bigoplus_{i} n_{i} \mathcal{R}_{i}\left(-y_{i}\right) \bigoplus_{j} n_{j} \mathcal{R}_{j}^{\pi}\left(-y_{j}\right)\right)-\mathcal{R}_{0}(\emptyset) 1
$$

For an explicit calculation of this expression, one uses the fact that

$$
\left(n_{i} \mathcal{R}_{i}\left(y_{i}\right)\right) \times\left(n_{j} \mathcal{R}_{j}\left(y_{j}\right)\right)=n_{i} n_{j} \bigoplus_{k=|i-j|}^{i+j} \mathcal{R}_{k}\left(y_{i}+y_{j}\right) \quad \text { with } k \text { integer and half-integer }
$$

and the same formula for $\mathcal{R}^{\pi}$ representations.

In the case of the orthosymplectic superalgebras, one considers the following decomposition of the $O S p(M \mid 2 n)$ fundamental representation:

$$
\mathbf{F}=\left(\bigoplus_{i} n_{i} \mathcal{R}_{i}\right) \oplus\left(\bigoplus_{j} n_{j} \mathcal{R}_{j}^{\pi}\right)
$$

which implies for the fundamental representations of $S O(M)$ and $S p(2 n)$ :

$$
\begin{aligned}
& \underline{M}=\left(\bigoplus_{i} n_{i} \mathcal{D}_{i}\right) \oplus\left(\bigoplus_{j} n_{j} \mathcal{D}_{j-1 / 2}\right) \\
& \underline{2 n}=\left(\bigoplus_{i} n_{i} \mathcal{D}_{i-1 / 2}\right) \oplus\left(\bigoplus_{j} n_{j} \mathcal{D}_{j}\right)
\end{aligned}
$$


For the $S O(M)$ part, one can introduce a non zero $U(1)$ eigenvalue $y_{i}$ only for representations $\mathcal{D}_{i}$ with $i$ integer, which appear twice and only twice. For the $S p(2 n)$ part, a non zero $U(1)$ eigenvalue $y_{i}$ is allowed only for representations $\mathcal{D}_{i}$ with $i$ half-integer, which appear twice and only twice.

For the superalgebra $\mathcal{G}$ itself, one has to group the $S l(2) \oplus U(1)$ representations $\mathcal{D}_{i}\left(y_{i}\right)$ into $O S p(1 \mid 2) \oplus U(1)$ representations $\mathcal{R}_{j}\left(y_{j}\right)=\mathcal{D}_{j}\left(y_{j}\right) \oplus \mathcal{D}_{j-1 / 2}\left(y_{j}\right)$. Therefore, if the decomposition of the $O S p(M \mid 2 n)$ fundamental representation $\mathbf{F}$ under a certain $O S p(1 \mid 2)$ is given by (11.6), non zero values $y$ of the $U(1)$ factor are allowed for the following combinations:

- the representation $\mathcal{R}_{i}$ appears twice and only twice $\left(n_{i}=2\right)$, and $i$ is integer, - the representation $\mathcal{R}_{i}^{\pi}$ appears twice and only twice $\left(n_{i}=2\right)$, and $i$ is halfinteger.

Moreover, $y$ can only take the values $0,1 / 4$ or $1 / 2$ if $i \neq 0$ (which lead to the values $0, \pm 1 / 2$ or \pm 1 for the $U(1)$ factor in the adjoint representation of $\mathcal{G}$ ). Finally, starting from a decomposition of the fundamental representation of $O S p(M \mid 2 n)$ under $O S p(1 \mid 2) \oplus U(1)$ of the form

$$
\mathbf{F}=\left(\bigoplus_{i} \mathcal{R}_{i}\left(y_{i}\right) \oplus \mathcal{R}_{i}\left(-y_{i}\right)\right) \oplus\left(\bigoplus_{j} \mathcal{R}_{j}^{\pi}\left(y_{j}\right) \oplus \mathcal{R}_{j}^{\pi}\left(-y_{j}\right)\right) \oplus\left(\bigoplus_{i, n_{i} \neq 2} n_{i} \mathcal{R}_{i}(0)\right) \oplus\left(\bigoplus_{j, n_{j} \neq 2} n_{j} \mathcal{R}_{j}^{\pi}(0)\right)
$$

the decomposition of the adjoint is given by

$$
\begin{aligned}
\mathbf{A d} & =\left(\bigoplus_{i} \mathcal{R}_{i}\left(y_{i}\right) \oplus \mathcal{R}_{i}\left(-y_{i}\right) \bigoplus_{i, n_{i} \neq 2} n_{i} \mathcal{R}_{i}(0)\right) \times\left.\left(\bigoplus_{i} \mathcal{R}_{i}\left(y_{i}\right) \oplus \mathcal{R}_{i}\left(-y_{i}\right) \underset{i, n_{i} \neq 2}{\bigoplus_{i}} n_{i} \mathcal{R}_{i}(0)\right)\right|_{A} \\
& \oplus\left(\bigoplus_{j} \mathcal{R}_{j}^{\pi}\left(y_{j}\right) \oplus \mathcal{R}_{j}^{\pi}\left(-y_{j}\right) \bigoplus_{j, n_{j} \neq 2} n_{j} \mathcal{R}_{j}^{\pi}(0)\right) \times\left.\left(\bigoplus_{j} \mathcal{R}_{j}^{\pi}\left(y_{j}\right) \oplus \mathcal{R}_{j}^{\pi}\left(-y_{j}\right) \underset{j, n_{j} \neq 2}{\bigoplus_{j}} n_{j} \mathcal{R}_{j}^{\pi}(0)\right)\right|_{S} \\
& \oplus\left(\bigoplus_{i} \mathcal{R}_{i}\left(y_{i}\right) \oplus \mathcal{R}_{i}\left(-y_{i}\right) \underset{i, n_{i} \neq 2}{\bigoplus_{i}} n_{i} \mathcal{R}_{i}(0)\right) \times\left(\bigoplus_{j} \mathcal{R}_{j}^{\pi}\left(y_{j}\right) \oplus \mathcal{R}_{j}^{\pi}\left(-y_{j}\right) \underset{j, n_{j} \neq 2}{\left.\bigoplus_{j} n_{j} \mathcal{R}_{j}^{\pi}(0)\right)}\right.
\end{aligned}
$$

The (anti)symmetric products of $\mathcal{R}$ representations are given by the formulae 10.18 and (10.22-10.29) modulo the following modifications due to the $U(1)$ eigenvalue:

$$
\begin{aligned}
\left(\mathcal{R}_{i}\left(y_{i}\right) \oplus \mathcal{R}_{i}\left(-y_{i}\right)\right) & \times\left.\left(\mathcal{R}_{i}\left(y_{i}\right) \oplus \mathcal{R}_{i}\left(-y_{i}\right)\right)\right|_{A}=\left(\mathcal{R}_{i} \times \mathcal{R}_{i}\right)_{A}\left(2 y_{i}\right) \oplus\left(\mathcal{R}_{i} \times \mathcal{R}_{i}\right)_{A}\left(-2 y_{i}\right) \\
& \oplus\left(\mathcal{R}_{i} \times \mathcal{R}_{i}\right)(0)
\end{aligned}
$$

and

$$
\begin{aligned}
\left(\mathcal{R}_{i}^{\pi}\left(y_{i}\right) \oplus \mathcal{R}_{i}^{\pi}\left(-y_{i}\right)\right) \times\left.\left(\mathcal{R}_{i}^{\pi}\left(y_{i}\right) \oplus \mathcal{R}_{i}^{\pi}\left(-y_{i}\right)\right)\right|_{S}=\left(\mathcal{R}_{i}^{\pi} \times \mathcal{R}_{i}^{\pi}\right)_{S}\left(2 y_{i}\right) \oplus\left(\mathcal{R}_{i}^{\pi} \times \mathcal{R}_{i}^{\pi}\right)_{S}\left(-2 y_{i}\right) \\
\oplus\left(\mathcal{R}_{i}^{\pi} \times \mathcal{R}_{i}^{\pi}\right)(0)
\end{aligned}
$$




\subsection{Superdefining vector}

The determination of the grading $H$ from the $O S p(1 \mid 2) \oplus U(1)$ decomposition of the fundamental representation is strictly the same as for the algebras case. One just has to "double the calculation" since the bosonic part of $\mathcal{G}$ is in general the direct sum of two simple algebras. Using the same basis for the Cartan algebras (see section 6.1), we will denote the defining vector as

$$
f=\left(f_{1}, \ldots, f_{n} ; f_{1}^{\prime}, \ldots, f_{n}^{\prime}\right)
$$

where $f_{i}$ refers to the first simple algebra and the $f_{i}^{\prime}$ to the second. For example, for the case of $S l(m \mid n)$ superalgebras, the contribution of a representation is:

$$
\begin{aligned}
& \mathcal{R}_{j}(y) \rightarrow\left(j+y, j-1+y, . .,-j+y, 0, . ., 0 ; j-\frac{1}{2}+y, j-\frac{3}{2}+y, . .,-j+\frac{1}{2}+y, 0, . ., 0\right) \\
& \mathcal{R}_{j}^{\pi}(y) \rightarrow\left(j-\frac{1}{2}+y, j-\frac{3}{2}+y, . .,-j+\frac{1}{2}+y, 0, . ., 0 ; j+y, j-1+y, . .,-j+y, 0, . ., 0\right)
\end{aligned}
$$

The other cases are analogous.

\section{$12 W$ superalgebras from Lie superalgebras of rank up to 4}

In the following tables, we present an exhaustive classification of super $W$ algebras arising from super Toda models based on classical superalgebras of rank up to 4 . The classification is listed in tables 10 to 17.

For the infinite series $\mathcal{G}=A(m, n)=S l(m+1 \mid n+1)$ with $m \neq n, A(n, n)=$ $S l(n+1 \mid n+1) / U(1), B(m, n)=O S p(2 m+1 \mid 2 n), C(n+1)=O S p(2 \mid 2 n)$ and $D(m, n)=O S p(2 m \mid 2 n)$, we give the decomposition of the fundamental representation of $\mathcal{G}$ with respect to $O S p(1 \mid 2) \oplus U(1)$, the minimal (i.e. the lowest dimensional) regular SSAs containing the $O S p(1 \mid 2)$ or (for the irregular cases) the corresponding singular embedding. Then, we give the superspin content with the same convention as for the bosonic tables. We recall that to a $W_{s}$ superfield correspond two fields $w_{s}$ and $w_{s+1 / 2}$. When the superspin is marked with a prime ('), the corresponding superfield $W_{s}$ has the "wrong" statistics (commuting fermions and anticommuting bosons). In the same column, we give under the superspin $s$ the hypercharge(s) $y$ when they exist.

For the two exceptional superalgebras $\mathcal{G}=G(3)$ and $F(4)$, we give the minimal regular SSA containing the $O S p(1 \mid 2)$ embedding, the decomposition of the adjoint representation of $\mathcal{G}$, and the superspin content. 


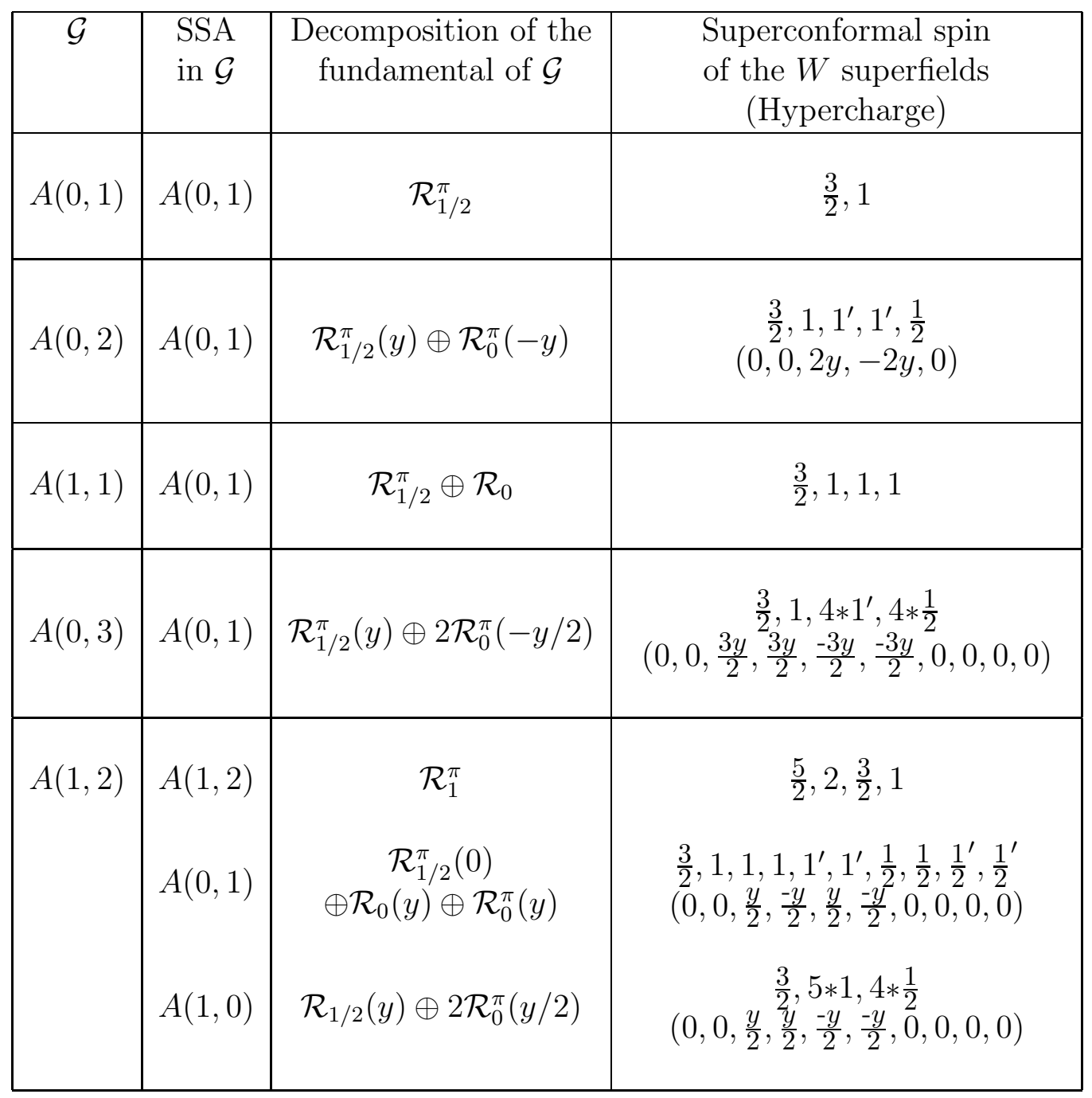

Table 10: $A(m, n)$ superalgebras up to rank 4 . 


\begin{tabular}{|c|c|c|c|}
\hline$\overline{\mathcal{G}}$ & $\begin{array}{l}\text { SSA } \\
\text { in } \mathcal{G}\end{array}$ & $\begin{array}{l}\text { Decomposition of the } \\
\text { fundamental of } \mathcal{G}\end{array}$ & $\begin{array}{c}\text { Superconformal spin } \\
\text { of the } W \text { superfields } \\
\text { (Hypercharge) }\end{array}$ \\
\hline$B(0,2)$ & $B(0,1)$ & $\mathcal{R}_{1 / 2}^{\pi} \oplus 2 \mathcal{R}_{0}^{\pi}$ & $\frac{3}{2}, 1^{\prime}, 1^{\prime}, \frac{1}{2}, \frac{1}{2}, \frac{1}{2}$ \\
\hline$B(1,1)$ & $\left.\begin{array}{c}B(1,1) \\
C(2) \\
B(0,1)\end{array}\right\}$ & $\begin{array}{c}\mathcal{R}_{1} \\
\mathcal{R}_{1 / 2}^{\pi} \oplus \mathcal{R}_{0}(y) \oplus \mathcal{R}_{0}(-y)\end{array}$ & $\begin{array}{c}2, \frac{3}{2} \\
\frac{3}{2}, 1,1, \frac{1}{2} \\
(0, y,-y, 0)\end{array}$ \\
\hline$B(0,3)$ & $B(0,1)$ & $\mathcal{R}_{1 / 2}^{\pi} \oplus 4 \mathcal{R}_{0}^{\pi}$ & $\frac{3}{2}, 1^{\prime}, 1^{\prime}, 1^{\prime}, 1^{\prime}, 10 * \frac{1}{2}$ \\
\hline$B(1,2)$ & $\left.\begin{array}{c}B(1,2) \\
B(1,1) \\
C(2) \\
B(0,1)\end{array}\right\}$ & $\begin{array}{c}\mathcal{R}_{3 / 2}^{\pi} \\
\mathcal{R}_{1} \oplus 2 \mathcal{R}_{0}^{\pi} \\
\mathcal{R}_{1 / 2}^{\pi} \oplus \mathcal{R}_{0}(y) \oplus \mathcal{R}_{0}(-y) \\
\oplus 2 \mathcal{R}_{0}^{\pi} \\
\mathcal{R}_{1 / 2}^{\pi}(y) \oplus \mathcal{R}_{1 / 2}^{\pi}(-y) \oplus \mathcal{R}_{0}\end{array}$ & $\begin{array}{c}\frac{7}{2}, 2, \frac{3}{2} \\
2, \frac{3}{2}, \frac{3^{\prime}}{2}, \frac{3^{\prime}}{2}, \frac{1}{2}, \frac{1}{2}, \frac{1}{2} \\
\frac{3}{2}, 1,1,1^{\prime}, 1^{\prime}, 4 * \frac{1}{2}, 4 * \frac{1^{\prime}}{2} \\
(0, y,-y, 6 * 0, y, y,-y,-y) \\
\frac{3}{2}, \frac{3}{2}, \frac{3}{2}, 1,1,1, \frac{1}{2} \\
(2 y,-2 y, 0, y,-y, 0,0)\end{array}$ \\
\hline$B(2,1)$ & $\left.\begin{array}{c}\left.\begin{array}{c}D(2,1) \\
B(1,1)\end{array}\right\} \\
C(2) \\
B(0,1)\end{array}\right\}$ & $\begin{array}{c}\mathcal{R}_{1} \oplus \mathcal{R}_{0}(y) \oplus \mathcal{R}_{0}(-y) \\
\mathcal{R}_{1 / 2}^{\pi} \oplus 4 \mathcal{R}_{0} \\
2 \mathcal{R}_{1 / 2} \oplus \mathcal{R}_{0}\end{array}$ & $\begin{array}{c}2, \frac{3}{2}, \frac{3}{2}, \frac{3}{2}, \frac{1}{2} \\
(0,2 y,-2 y, 0,0) \\
\frac{3}{2}, 4 * 1,6 * \frac{1}{2} \\
\frac{3}{2}, 1,1,1,1^{\prime}, 1^{\prime}, \frac{1}{2}, \frac{1}{2}, \frac{1}{2}\end{array}$ \\
\hline
\end{tabular}

Table 11: $B(m, n)$ superalgebras of rank 2 and 3. 


\begin{tabular}{|c|c|c|c|}
\hline $\mathcal{G}$ & $\begin{array}{l}\text { SSA } \\
\text { in } \mathcal{G}\end{array}$ & $\begin{array}{l}\text { Decomposition of the } \\
\text { fundamental of } \mathcal{G}\end{array}$ & $\begin{array}{c}\text { Superconformal spin } \\
\text { of the } W \text { superfields } \\
\text { (Hypercharge) }\end{array}$ \\
\hline$B(0,4)$ & $B(0,1)$ & $\mathcal{R}_{1 / 2}^{\pi} \oplus 6 \mathcal{R}_{0}^{\pi}$ & $\frac{3}{2}, 6 * 1^{\prime}, 21 * \frac{1}{2}$ \\
\hline$B(1,3)$ & $\begin{array}{c}B(1,2) \\
\left.\begin{array}{c}B(1,1) \\
C(2) \oplus B(0,1) \\
A(0,1)\end{array}\right\} \\
\left.\begin{array}{c}C(2) \\
B(0,1)\end{array}\right\}\end{array}$ & $\begin{array}{c}\mathcal{R}_{3 / 2}^{\pi} \oplus 2 \mathcal{R}_{0}^{\pi} \\
\mathcal{R}_{1} \oplus 4 \mathcal{R}_{0}^{\pi} \\
\mathcal{R}_{1 / 2}^{\pi}(y) \oplus \mathcal{R}_{1 / 2}^{\pi}(-y) \\
\oplus \mathcal{R}_{0} \oplus 2 \mathcal{R}_{0}^{\pi} \\
\mathcal{R}_{1 / 2}^{\pi} \oplus 4 \mathcal{R}_{0}^{\pi} \\
\oplus \mathcal{R}_{0}(y) \oplus \mathcal{R}_{0}(-y)\end{array}$ & $\begin{array}{c}\frac{7}{2}, 2,2^{\prime}, 2^{\prime}, \frac{3}{2}, \frac{1}{2}, \frac{1}{2}, \frac{1}{2} \\
2, \frac{3}{2}, \frac{3}{2}^{\prime}, \frac{3}{2}^{\prime}, \frac{3}{2}^{\prime}, \frac{3}{2}^{\prime}, 10 * \frac{1}{2} \\
3 * \frac{3}{2}, 1,1^{\prime}, 1^{\prime}, 1,1^{\prime}, 1^{\prime}, 1,4 * \frac{1}{2}, 2 * \frac{1}{2}^{\prime} \\
(2 y,-2 y, 0,3 * y, 3 *-y, 7 * 0) \\
\frac{3}{2}, 2 * 1,4 * 1^{\prime}, 11 * \frac{1}{2}, 9 * \frac{1}{2}^{\prime} \\
(0, y,-y, 15 * 0,4 * y, 4 *-y, 0)\end{array}$ \\
\hline$B(2,2)$ & $\begin{array}{c}\left.\begin{array}{c}B(2,2) \\
D(2,2) \\
B(1,2)\end{array}\right\} \\
\left.\begin{array}{c}D(2,1) \\
B(1,1)\end{array}\right\} \\
\left.\begin{array}{c}D(2,1) \oplus B(0,1) \\
B(1,1) \oplus C(2) \\
C(2) \oplus C(2) \\
A(0,1) \\
C(2) \\
B(0,1)\end{array}\right\} \\
A(1,0)\end{array}$ & $\begin{array}{c}\mathcal{R}_{2} \\
\mathcal{R}_{3 / 2}^{\pi} \oplus \mathcal{R}_{0}(y) \oplus \mathcal{R}_{0}(-y) \\
\mathcal{R}_{1} \oplus 2 \mathcal{R}_{0}^{\pi} \\
\oplus \mathcal{R}_{0}(y) \oplus \mathcal{R}_{0}(-y) \\
\mathcal{R}_{1} \oplus \mathcal{R}_{1 / 2}^{\pi} \oplus \mathcal{R}_{0} \\
\mathcal{R}_{1 / 2}^{\pi}(y) \oplus \mathcal{R}_{1 / 2}^{\pi}(-y) \\
\oplus 3 \mathcal{R}_{0} \\
\mathcal{R}_{1 / 2}^{\pi} \oplus 4 \mathcal{R}_{0} \oplus 2 \mathcal{R}_{0}^{\pi} \\
2 \mathcal{R}_{1 / 2} \oplus \mathcal{R}_{0} \oplus 2 \mathcal{R}_{0}^{\pi}\end{array}$ & $\begin{array}{c}4, \frac{7}{2}, 2, \frac{3}{2} \\
\frac{7}{2}, 2,2,2, \frac{3}{2}, \frac{1}{2} \\
(0, y,-y, 0,0,0) \\
2, \frac{3}{2}, \frac{3}{2}, \frac{3}{2}, \frac{3}{2}^{\prime}, \frac{3}{2}^{\prime}, 4 * \frac{1}{2}, 4 * \frac{1}{2}^{\prime} \\
(0,2 y,-2 y, 0,6 * 0, y, y,-y,-y) \\
2,2, \frac{3}{2}, \frac{3}{2}, \frac{3}{2}, \frac{3}{2}, 1,1 \\
\frac{3}{2}, \frac{3}{2}, \frac{3}{2}, 7 * 1, \frac{1}{2}, \frac{1}{2}, \frac{1}{2}, \frac{1}{2}^{\prime} \\
(2 y,-2 y, 0,3 * y, 3 *-y, 0,4 * 0) \\
\frac{3}{2}, 4 * 1,2 * 1^{\prime}, 9 * \frac{1}{2}, 8 * \frac{1}{2}^{\prime} \\
\frac{3}{2}, 7 * 1,1^{\prime}, 1^{\prime}, 6 * \frac{1}{2}, 2 * \frac{1}{2}^{\prime}\end{array}$ \\
\hline$B(3,1)$ & $\begin{array}{c}\left.\begin{array}{c}D(2,1) \\
B(1,1)\end{array}\right\} \\
\left.\begin{array}{c}C(2) \\
B(0,1)\end{array}\right\} \\
A(1,0)\end{array}$ & $\begin{array}{c}\mathcal{R}_{1} \oplus 4 \mathcal{R}_{0} \\
\mathcal{R}_{1 / 2}^{\pi} \oplus 6 \mathcal{R}_{0} \\
2 \mathcal{R}_{1 / 2} \oplus 3 \mathcal{R}_{0}\end{array}$ & $\begin{array}{c}2,5 * \frac{3}{2}, 6 * \frac{1}{2} \\
\frac{3}{2}, 6 * 1,15 * \frac{1}{2} \\
\frac{3}{2}, 3 * 1,6 * 1^{\prime}, 6 * \frac{1}{2}\end{array}$ \\
\hline
\end{tabular}




\begin{tabular}{|c|c|c|c|}
\hline$\overline{\mathcal{G}}$ & $\begin{array}{l}\mathrm{SSA} \\
\text { in } \mathcal{G}\end{array}$ & $\begin{array}{l}\text { Decomposition of the } \\
\text { fundamental of } \mathcal{G}\end{array}$ & $\begin{array}{c}\text { Superconformal spin } \\
\text { of the } W \text { superfields } \\
\text { (Hypercharge) }\end{array}$ \\
\hline$D(2,1)$ & $\begin{array}{c}D(2,1) \\
C(2) \\
A(1,0)\end{array}$ & $\begin{array}{c}\mathcal{R}_{1} \oplus \mathcal{R}_{0} \\
\mathcal{R}_{1 / 2}^{\pi} \oplus 3 \mathcal{R}_{0} \\
2 \mathcal{R}_{1 / 2}\end{array}$ & $\begin{array}{c}2, \frac{3}{2}, \frac{3}{2} \\
\frac{3}{2}, 1,1,1, \frac{1}{2}, \frac{1}{2}, \frac{1}{2} \\
\frac{3}{2}, 1,1,1, \frac{1}{2}, \frac{1}{2}, \frac{1}{2}\end{array}$ \\
\hline$D(2,2)$ & 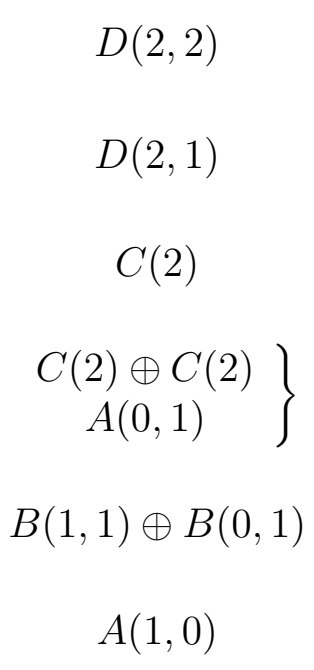 & $\begin{array}{c}\mathcal{R}_{3 / 2}^{\pi} \oplus \mathcal{R}_{0} \\
\mathcal{R}_{1} \oplus \mathcal{R}_{0} \oplus 2 \mathcal{R}_{0}^{\pi} \\
\mathcal{R}_{1 / 2}^{\pi} \oplus 3 \mathcal{R}_{0} \oplus 2 \mathcal{R}_{0}^{\pi} \\
\mathcal{R}_{1 / 2}^{\pi} \oplus \mathcal{R}_{0}(y) \oplus \mathcal{R}_{0}(-y) \\
\mathcal{R}_{1} \oplus \mathcal{R}_{1 / 2}^{\pi} \\
2 \mathcal{R}_{1 / 2} \oplus 2 \mathcal{R}_{0}^{\pi}\end{array}$ & $\begin{array}{c}\frac{7}{2}, 2,2, \frac{3}{2} \\
2, \frac{3}{2}, \frac{3}{2}, \frac{3^{\prime}}{2}, \frac{3^{\prime}}{2}, 5 * \frac{1}{2} \\
\frac{3}{2}, 1,1,1,1^{\prime}, 1^{\prime}, 6 * \frac{1}{2}, 6 * \frac{1}{2}^{\prime} \\
\frac{3}{2}, \frac{3}{2}, \frac{3}{2}, 5 * 1, \frac{1}{2}, \frac{1}{2} \\
(3 * 0, y,-y, 5 * 0) \\
2,2, \frac{3}{2}, \frac{3}{2}, \frac{3}{2}, 1 \\
\frac{3}{2}, 7 * 1,6 * \frac{1}{2}\end{array}$ \\
\hline$D(3,1)$ & $\begin{array}{c}D(2,1) \\
C(2) \\
A(1,0)\end{array}$ & $\begin{array}{c}\mathcal{R}_{1} \oplus 3 \mathcal{R}_{0} \\
\mathcal{R}_{1 / 2}^{\pi} \oplus 5 \mathcal{R}_{0} \\
2 \mathcal{R}_{1 / 2} \oplus 2 \mathcal{R}_{0}\end{array}$ & $\begin{array}{c}2,4 * \frac{3}{2}, \frac{1}{2}, \frac{1}{2}, \frac{1}{2} \\
\frac{3}{2}, 5 * 1,10 * \frac{1}{2} \\
\frac{3}{2}, 1,1,1,4 * 1^{\prime}, 4 * \frac{1}{2}\end{array}$ \\
\hline
\end{tabular}

Table 13: $D(m, n)$ superalgebras up to rank 4. 


\begin{tabular}{|c|c|c|c|}
\hline $\mathcal{G}$ & $\begin{array}{l}\text { SSA } \\
\text { in } \mathcal{G}\end{array}$ & $\begin{array}{l}\text { Decomposition of the } \\
\text { fundamental of } \mathcal{G}\end{array}$ & $\begin{array}{l}\text { Superconformal spin } \\
\text { of the } W \text { superfields } \\
\text { (Hypercharge) }\end{array}$ \\
\hline$C(3)$ & $\begin{array}{l}A(0,1) \\
C(2)\end{array}$ & $\begin{array}{c}\mathcal{R}_{1 / 2}^{\pi}(y) \oplus \mathcal{R}_{1 / 2}^{\pi}(-y) \\
\mathcal{R}_{1 / 2}^{\pi} \oplus \mathcal{R}_{0} \oplus 2 \mathcal{R}_{0}^{\pi}\end{array}$ & $\begin{array}{c}\frac{3}{2}, \frac{3}{2}, \frac{3}{2}, 1, \frac{1}{2} \\
(0,2 y,-2 y, 0,0) \\
\frac{3}{2}, 1,1^{\prime}, 1^{\prime}, \frac{1}{2}, \frac{1}{2}, \frac{1}{2}, \frac{1}{2}^{\prime}, \frac{1}{2}^{\prime}\end{array}$ \\
\hline$C(4)$ & $\begin{array}{l}A(0,1) \\
C(2)\end{array}$ & $\begin{array}{c}\mathcal{R}_{1 / 2}^{\pi}(y) \oplus \mathcal{R}_{1 / 2}^{\pi}(-y) \oplus 2 \mathcal{R}_{0}^{\pi} \\
\mathcal{R}_{1 / 2}^{\pi} \oplus \mathcal{R}_{0} \oplus 4 \mathcal{R}_{0}^{\pi}\end{array}$ & $\begin{array}{c}3 * \frac{3}{2}, 1,4 * 1^{\prime}, 4 * \frac{1}{2} \\
(0,2 y,-2 y, 0, y, y,-y,-y, 4 * 0) \\
\frac{3}{2}, 1,4 * 1^{\prime}, 10 * \frac{1}{2}, 4 * \frac{1}{2}^{\prime}\end{array}$ \\
\hline
\end{tabular}

Table 14: $C(n+1)$ superalgebras up to rank 4 .

\begin{tabular}{|c|c|c|}
\hline SSA & $\begin{array}{c}O S p(1 \mid 2) \text { decomposition } \\
\text { of } G(3)\end{array}$ & $\begin{array}{c}\text { Superconformal spin } \\
\text { of the } W \text { superfields }\end{array}$ \\
\hline$A(1,0)$ & $\mathcal{R}_{1} \oplus 3 \mathcal{R}_{1 / 2} \oplus 4 \mathcal{R}_{1 / 2}^{\prime} \oplus 3 \mathcal{R}_{0} \oplus 2 \mathcal{R}_{0}^{\prime}$ & $\frac{3}{2}, 1,1,1,4 * 1^{\prime}, \frac{1}{2}, \frac{1}{2}, \frac{1}{2}, \frac{1}{2}^{\prime}, \frac{1}{2}^{\prime}$ \\
\hline$A(1,0)^{\prime}$ & $2 \mathcal{R}_{3 / 2}^{\prime} \oplus \mathcal{R}_{1} \oplus 3 \mathcal{R}_{1 / 2} \oplus 3 \mathcal{R}_{0}$ & $2^{\prime}, 2^{\prime}, \frac{3}{2}, 1,1,1, \frac{1}{2}, \frac{1}{2}, \frac{1}{2}$ \\
\hline$B(0,1)$ & $\mathcal{R}_{1} \oplus 6 \mathcal{R}_{1 / 2} \oplus 8 \mathcal{R}_{0}$ & $\frac{3}{2}, 6 * 1,8 * \frac{1}{2}$ \\
\hline$B(1,1)$ & $\mathcal{R}_{3 / 2} \oplus 2 \mathcal{R}_{3 / 2}^{\prime} \oplus \mathcal{R}_{1} \oplus 3 \mathcal{R}_{0} \oplus 2 \mathcal{R}_{0}^{\prime}$ & $2,2^{\prime}, 2^{\prime}, \frac{3}{2}, \frac{1}{2}, \frac{1}{2}, \frac{1}{2}, \frac{1^{\prime}}{2}, \frac{1^{\prime}}{2}$ \\
\hline \multicolumn{2}{|c|}{$\mathcal{R}_{2} \oplus \mathcal{R}_{3 / 2} \oplus 3 \mathcal{R}_{1}$} & $\frac{5}{2}, 2, \frac{3}{2}, \frac{3}{2}, \frac{3}{2}$ \\
\hline
\end{tabular}

Table 15: The exceptional superalgebra $G(3)$. 


\begin{tabular}{|c|c|c|}
\hline SSA & $\begin{array}{c}O S p(1 \mid 2) \text { decomposition } \\
\text { of } F(4)\end{array}$ & $\begin{array}{c}\text { Superconformal spin } \\
\text { of the } W \text { superfields }\end{array}$ \\
\hline$A(1,0)$ & $\mathcal{R}_{1} \oplus 7 \mathcal{R}_{1 / 2} \oplus 14 \mathcal{R}_{0}$ & $\frac{3}{2}, 7 * 1,14 * \frac{1}{2}$ \\
\hline$A(0,1)$ & $\mathcal{R}_{1} \oplus 3 \mathcal{R}_{1 / 2} \oplus 6 \mathcal{R}_{1 / 2}^{\prime} \oplus 6 \mathcal{R}_{0} \oplus 2 \mathcal{R}_{0}^{\prime}$ & $\frac{3}{2}, 3 * 1,6 * 1^{\prime}, 6 * \frac{1}{2}, \frac{1}{2}^{\prime}, \frac{1}{2}^{\prime}$ \\
\hline$C(2)$ & $5 \mathcal{R}_{1} \oplus 3 \mathcal{R}_{1 / 2} \oplus 6 \mathcal{R}_{0}$ & $5 * \frac{3}{2}, 3 * 1,6 * \frac{1}{2}$ \\
\hline$D(2,1 ; 2)$ & $\mathcal{R}_{3 / 2} \oplus 2 \mathcal{R}_{3 / 2}^{\prime} \oplus 2 \mathcal{R}_{1} \oplus 2 \mathcal{R}_{1 / 2}^{\prime} \oplus 3 \mathcal{R}_{0}$ & $2,2^{\prime}, 2^{\prime}, \frac{3}{2}, \frac{3}{2}, 1^{\prime}, 1^{\prime}, \frac{1}{2}, \frac{1}{2}, \frac{1}{2}$ \\
\hline
\end{tabular}

Table 16: The exceptional superalgebra $F(4)$.

\begin{tabular}{|c|c|c|}
\hline SSA & $\begin{array}{c}\text { Decomposition of the } \\
\text { fundamental of } D(2,1 ; \alpha)\end{array}$ & $\begin{array}{c}\text { Superconformal spin } \\
\text { of the } W \text { superfields }\end{array}$ \\
\hline$D(2,1)$ & $\mathcal{R}_{1} \oplus \mathcal{R}_{0}$ & $2, \frac{3}{2}, \frac{3}{2}$ \\
\hline$C(2)$ & $\mathcal{R}_{1 / 2}^{\pi} \oplus 3 \mathcal{R}_{0}$ & $\frac{3}{2}, 1,1,1, \frac{1}{2}, \frac{1}{2}, \frac{1}{2}$ \\
\hline$A(1,0)$ & $2 \mathcal{R}_{1 / 2}$ & $\frac{3}{2}, 1,1,1, \frac{1}{2}, \frac{1}{2}, \frac{1}{2}$ \\
\hline
\end{tabular}

Table 17: The exceptional superalgebra $D(2,1 ; \alpha)$. 


\section{Quadratic-, Quasi- and $Z_{2} \times Z_{2}$ - Supercon- formal algebras}

We have a natural framework to study superconformal algebras. Let us first recall that a quadratic-superconformal algebra is a Zamolodchikov superalgebra made of one spin 2 field corresponding to $T(x)$ (and forming a Virasoro algebra), $N$ fermionic supersymmetry charges $G^{\alpha}(x)$ which are spin $\frac{3}{2}$ primary fields with respect to $T(x)$, and a Kac-Moody (KM) algebra (i.e. spin 1 primary fields). The spin $\frac{3}{2}$ generators are required to form a representation of the KM algebra, but the quadratic-superconformal superalgebra is not (in general) a Lie superalgebra in the sense that the $\mathrm{PB}\left\{G^{\alpha}(x), G^{\beta}\left(x^{\prime}\right)\right\}_{P B}$ contains quadratic terms in the KM currents [16, 19].

The "usual" superconformal algebras, i.e. the Ademollo et al. algebras 20] and the one parameter algebra found in [21], are the only closed Lie superconformal algebras we know. We will refer to them as Lie superconformal algebras and called the corresponding supersymmetries "true" supersymmetries.

The same definition holds for a quasi-superconformal algebra [16], except that its spin $\frac{3}{2}$ fields $G^{\alpha}(x)$ are bosonic ("wrong" statistics). As an example, the algebra explicited in section 7.3 , possessing two spin- $\frac{3}{2}$ and one spin-1 fields, is quasi-superconformal.

An algebra with both bosonic and fermionic spin $\frac{3}{2}$ currents is called $\mathbb{Z}_{2} \times \mathbb{Z}_{2}$ superconformal algebra. In that case, spin 1 fermions may also appear.

It should be clear to the reader that the part \ contains all the tools necessary for the determination of the quasi-superconformal algebras, whereas the quadratic and $\mathbb{Z}_{2} \times \mathbb{Z}_{2}$ superconformal algebras can be obtained from the part $\mathbb{I}$. Note however, that the supersymmetric treatment we have used (and which makes naturally appear a $N=1$ Lie superconformal algebra) leads to the emergence of spin $\frac{1}{2}$ fields. As it is now well-known, to avoid these fermions, one can factorize them [22]. These algebras (without spin $\frac{1}{2}$ fermions) have already been classified at the quantum level in [16]. We show hereafter that all the algebras of [16] can be realized at the classical level as symmetries of Toda models. Moreover, two new (with respect to [16]) $\mathbb{Z}_{2} \times \mathbb{Z}_{2}$ superconformal algebras can be identified from the study of $G(3)$ and $F(4)$.

\subsection{Quasi-superconformal algebras}

From the study of part \, we can see that such algebras, with only one spin 2 and no spin $s>2$, are obtained when the fundamental representation of $\operatorname{Sl}(n)$ and $S p(2 n)$ (resp. $S O(n)$ ) algebras contains only one (resp. two) $\mathcal{D}_{1 / 2}$ representation(s). This means that we are reducing these Lie algebras with respect to a regular $A_{1}$. Using the results of part [and [17] for the exceptional algebras $E_{6,7,8}$, 
we obtain the classification of table 18 .

\subsection{Quadratic-superconformal algebras}

They are obtained from the reduction of a superalgebra with respect to an $O S p(1 \mid 2)$ SSA. Note that "wrong" statistic superfields may appear and lead to $\mathbb{Z}_{2} \times \mathbb{Z}_{2}$ superconformal algebras. From the rules given in section 10.2.1, relating $\mathcal{R}^{\prime}$ representations of the adjoint, to $\mathcal{R}$ and $\mathcal{R}^{\pi}$ representations of the fundamental, it is easy to compute the allowed reductions. As an example, let us study the $S l(m \mid n)$ algebras: the reduction with respect to $S l(1 \mid 2)$ reads $\underline{n+m}=\mathcal{R}_{1 / 2}^{\pi}+(m-1) \mathcal{R}_{0}+(n-2) \mathcal{R}_{0}^{\pi}$, so that we must set $n=2$ to avoid "wrong" statistics. Thus, only the $S l(n \mid 2)$ (or $S l(2 \mid n)$ ) algebra leads to quadraticsuperconformal algebras. The same calculation leads to the list:

$$
S l(n \mid 2) \quad, \quad O S p(4 \mid 2 n) \quad, \quad O S p(n \mid 2) \quad, \quad F(4) \quad, \quad G(3)
$$

We summarize the results in the table 19. Note that the regular superalgebra which characterizes the $O S p(1 \mid 2)$, provides the number $N_{0}$ of "true" supersymmetries of the $W$ algebra: $N_{0}=1$ for a regular $O S p(1 \mid 2), N_{0}=2$ for the superprincipal $O S p(1 \mid 2)$ of $S l(1 \mid 2)$ and $O S p(2 \mid 2), N_{0}=3$ if the previous $S l(1 \mid 2)$ or $O S p(2 \mid 2)$ can be embedded in an $O S p(3 \mid 2) \mathrm{SSA}$, and $N_{0}=4$ if the $S l(2 \mid 1)$ or $O S p(2 \mid 2)$ is contained in $O S p(4 \mid 2)$ or $D(2,1 ; \alpha)$ SSAs.

\section{3 $Z_{2} \times Z_{2}$ superconformal algebras}

Their classification is easily deduced from the previous section. We begin with the $\mathbb{Z}_{2} \times \mathbb{Z}_{2}$ superconformal algebras that do not contain superspin $\frac{1}{2}$ bosons, so that we can define a (right statistic) super-KM algebra. These algebras are listed in table 20 .

If now one introduces the superspin $\frac{1}{2}$ bosons, the number of allowed superalgebras is much larger. In fact, in accordance with [16], we find one (resp. two) $\mathbb{Z}_{2} \times \mathbb{Z}_{2}$ superconformal algebras from each $A(m, n)$ and $C(n+1)$ (resp. $B(m, n)$ and $D(m, n))$ superalgebras. However, for $F(4)$ and $G(3)$, we find two new $\mathbb{Z}_{2} \times \mathbb{Z}_{2}$ superconformal algebras, different from the two quadraticsuperconformal algebras of [16], already listed in table 19. This seems to indicate that these two algebras exist only at the classical level. The results are summarized in table 21 . 


\begin{tabular}{|c|c|c|c|}
\hline $\begin{array}{c}\text { Algebra } \\
\mathcal{G}\end{array}$ & $\begin{array}{c}\text { Decomposition of } \\
\text { the fundamental of } \mathcal{G}\end{array}$ & $\begin{array}{c}\text { Conformal spin } \\
\text { of the } W \text { generators }\end{array}$ & $\begin{array}{c}\text { Residual Kac-Moody } \\
\text { algebra }\end{array}$ \\
\hline$S l(n)$ & $\underline{n}=\mathcal{D}_{1 / 2} \oplus(n-2) \mathcal{D}_{0}$ & $\begin{array}{c}2,2(n-2) * \frac{3}{2}, \\
(n-2))^{2} * 1\end{array}$ & $S l(n-2) \oplus U(1)$ \\
\hline$S O(n)$ & $\underline{n}=2 \mathcal{D}_{1 / 2}+(n-4) \mathcal{D}_{0}$ & $\begin{array}{c}2,2(n-4) * \frac{3}{2}, \\
{\left[\frac{(n-4)(n-5)}{2}+3\right] * 1}\end{array}$ & $S O(n-4) \oplus S l(2)$ \\
\hline$S p(2 n)$ & $\underline{2 n}=\mathcal{D}_{1 / 2}+(2 n-2)$ & $\begin{array}{c}2,(2 n-4) * \frac{3}{2}, \\
(n-2)(2 n-3) * 1\end{array}$ & $S p(2 n-2)$ \\
\hline$G_{2}$ & $\underline{7}=2 \mathcal{D}_{1 / 2}+3 \mathcal{D}_{0}$ & $2, \frac{3}{2}, \frac{3}{2}, \frac{3}{2}, \frac{3}{2}, 1,1,1$ & $S l(2)$ \\
\hline$F_{4}$ & $\underline{26}=10 \mathcal{D}_{1 / 2}+6 \mathcal{D}_{0}$ & $2,18 * \frac{3}{2}, 13 * 1$ & $S p(6)$ \\
\hline$E_{6}$ & $\underline{27}=6 \mathcal{D}_{1 / 2}+15 \mathcal{D}_{0}$ & $2,20 * \frac{3}{2}, 35 * 1$ & $S l(6)$ \\
\hline$E_{7}$ & $\underline{56}=12 \mathcal{D}_{1 / 2}+32 \mathcal{D}_{0}$ & $2,32 * \frac{3}{2}, 66 * 1$ & $S O(12)$ \\
\hline$E_{8}$ & $\underline{248}=\mathcal{D}_{1}+56 \mathcal{D}_{1 / 2}+133 \mathcal{D}_{0}$ & $2,56 * \frac{3}{2}, 133 * 1$ & $E_{7}$ \\
\hline
\end{tabular}

Table 18: Classification of quasi-superconformal algebras. 


\begin{tabular}{|c|c|c|c|c|}
\hline $\mathcal{G}$ & $\begin{array}{c}\text { Min. includ. } \\
\text { regular SSA }\end{array}$ & $N_{0}$ & $\begin{array}{c}\text { Superconformal spin } \\
\text { of the } W \text { generators }\end{array}$ & $\begin{array}{c}\text { Super KM } \\
\text { algebra }\end{array}$ \\
\hline$A(1, n)$ & $A(1,0)$ & 2 & $\frac{3}{2},(2 n+1) * 1, n^{2} * \frac{1}{2}$ & $A_{n-1} \oplus U(1)$ \\
\hline$D(2, n)$ & $A(1,0)$ & 4 & $\frac{3}{2},(4 n-1) * 1,[(n-1)(2 n-1)+3] * \frac{1}{2}$ & $C_{n-1} \oplus 3 U(1)$ \\
\hline$D(m, 1)$ & $C(2)$ & 4 & $\frac{3}{2},(2 m-1) * 1,(m-1)(2 m-1) * \frac{1}{2}$ & $B_{m-1}$ \\
\hline$B(m, 1)$ & $C(2)$ & $\left\{\begin{array}{l}3(m=1) \\
4(m>1)\end{array}\right.$ & $\frac{3}{2}, 2 m * 1, m(2 m-1) * \frac{1}{2}$ & $D_{m}$ \\
\hline$G(3)$ & $B(0,1)$ & 1 & $\frac{3}{2}, 6 * 1,8 * \frac{1}{2}$ & $A_{2}$ \\
\hline$F(4)$ & $A(1,0)$ & 2 & $\frac{3}{2}, 7 * 1,14 * \frac{1}{2}$ & $G_{2}$ \\
\hline
\end{tabular}

Table 19: Quadratic-superconformal algebras.

\begin{tabular}{|c|c|c|c|c|}
\hline $\mathcal{G}$ & $\begin{array}{c}\text { Min. includ. } \\
\text { regular SSA }\end{array}$ & $N_{0}$ & $\begin{array}{c}\text { Superconformal spin } \\
\text { of the } W \text { generators }\end{array}$ & $\begin{array}{c}\text { Super KM } \\
\text { algebra }\end{array}$ \\
\hline$A(1, n)$ & $A(1,0)$ & 2 & $\frac{3}{2}, 1,2 n * 1^{\prime}, n^{2} * \frac{1}{2}$ & $A_{n-1} \oplus U(1)$ \\
\hline$D(m, 1)$ & $A(1,0)$ & 4 & $\frac{3}{2}, 3 * 1,4(m-2) * 1^{\prime},[(m-2)(2 m-5)+3] * \frac{1}{2}$ & $D_{m} \oplus 3 U(1)$ \\
\hline$B(m, 1)$ & $A(1,0)$ & 4 & $\frac{3}{2}, 3 * 1,2(2 m-3) * 1^{\prime},[(m-2)(2 m-3)+3] * \frac{1}{2}$ & $B_{m-2} \oplus 3 U(1)$ \\
\hline$B(0, n)$ & $B(0,1)$ & 1 & $\frac{3}{2},(2 n-2) * 1^{\prime},(n-1)(2 n-1) * \frac{1}{2}$ & $B_{n-1}$ \\
\hline
\end{tabular}

Table 20: $\mathbb{Z}_{2} \times \mathbb{Z}_{2}$ superconformal algebras (no superspin $\frac{1}{2}$ bosonic superfield). 


\begin{tabular}{|c|c|c|c|}
\hline $\mathcal{G}$ & $\begin{array}{l}\text { Min. includ. } \\
\text { regular SSA }\end{array}$ & $N_{0}$ & $\begin{array}{l}\text { Superconformal spin } \\
\text { of the } W \text { generators }\end{array}$ \\
\hline$A(m, n)$ & $A(1,0)$ & 2 & $\begin{array}{c}\frac{3}{2},(2 m-1) * 1,2 n * 1^{\prime}, \\
{\left[(m-1)^{2}+n^{2}\right] * \frac{1}{2}, 2(m-1) n * \frac{1}{2}^{\prime}}\end{array}$ \\
\hline$D(m, n)$ & $\begin{array}{c}C(2) \\
A(1,0)\end{array}$ & 4 & $\begin{array}{c}\frac{3}{2},(2 m-1) * 1,(2 n-2) * 1^{\prime}, \\
{[(m-1)(2 m-1)+(n-1)(2 n-1)] * \frac{1}{2},} \\
(2 m-1)(2 n-2) * \frac{1}{2}^{\prime} \\
\frac{3}{2},(4 n-1) * 1,4(m-2) * 1^{\prime}, \\
{[(m-2)(2 m-5)+(n-1)(2 n-1)+3] * \frac{1}{2},} \\
4(m-2)(n-1) * \frac{1}{2}^{\prime}\end{array}$ \\
\hline$B(m, n)$ & $C(2)$ & $\left\{\begin{array}{l}3(m=1) \\
4(m>1)\end{array}\right.$ & $\begin{array}{c}\frac{3}{2}, 2 m * 1,(2 n-2) * 1^{\prime}, \\
{[m(2 m-1)+(n-1)(2 n-1)] * \frac{1}{2},} \\
4 m(n-1) * \frac{1}{2}^{\prime} \\
\frac{3}{2},(4 n-1) * 1,2(2 m-3) * 1^{\prime}, \\
{[(m-2)(2 m-3)+(n-1)(2 n-1)+3] * \frac{1}{2},} \\
2(2 m-3)(n-1) * \frac{1}{2}^{\prime}\end{array}$ \\
\hline$C(n+1)$ & $C(2)$ & 2 & $\begin{array}{c}\frac{3}{2}, 1,(2 n-2) * 1^{\prime}, \\
(n-1)(2 n-1) * \frac{1}{2},(2 n-2) * \frac{1}{2}^{\prime}\end{array}$ \\
\hline$G(3)$ & $A(1,0)$ & 4 & $\frac{3}{2}, 3 * 1,4 * 1^{\prime}, 3 * \frac{1}{2}, 2 * \frac{1^{\prime}}{2}$ \\
\hline$F(4)$ & $A(0,1)$ & 4 & $\frac{3}{2}, 3 * 1,6 * 1^{\prime}, 6 * \frac{1}{2}, 2 * \frac{1^{\prime}}{2}$ \\
\hline
\end{tabular}

Table 21: $\mathbb{Z}_{2} \times \mathbb{Z}_{2}$ superalgebras (with superspin $\frac{1}{2}$ bosons). 


\section{Conclusion}

In the classification we have obtained, each $W$ (super)algebra is characterized by its (super)conformal spin content and the couple $(S l(2), \mathcal{G})$ if $\mathcal{G}$ is a simple Lie algebra, respectively $(O S p(1 \mid 2), \mathcal{G})$ if $\mathcal{G}$ is a Lie superalgebra. The PB of the corresponding $W$ (super)algebra can then be determined via the general method reminded in section 2.1. However, rather important simplifications occur when the $U(1)$ factor commuting with $S l(2)$, resp. $O S p(1 \mid 2)$, exists: the admitted $Y$ values are also provided in our tables.

It has seemed to us necessary to reconsider in a first step the problem of the $S l(2)$ subalgebras in a simple Lie algebra $\mathcal{G}$, in order to make explicit our results in the algebraic case, and also to propose the generalization we have obtained for the supersymmetric one. We hope that the tables in which are gathered our results are presented in an enough convenient way to allow a direct use. This has been at least the case for us to easily recognize the superconformal algebras of [16].

Among the different problems one can immediately think of, an urgent one is of course the quantum case. Some interesting works [19, 23, 24, 25, 26] already exist, but a general treatment would be necessary. Another question we wish we could answer is how large is the class of $W$ (super)algebras which are symmetries of Toda theories, in the complete set of $W$ algebras.

\section{Acknowledgements}

It is a pleasure to thank A. Deckmyn, F. Delduc, K. Hornfeck and A. Saveliev for fruitful discussions. 


\section{References}

[1] A. Bilal and J.L. Gervais, Phys. Lett. 206B (1988) 412;

Nucl. Phys. B314 (1989) 646; ibid B318 (1989) 579.

[2] O. Babelon, Phys. Lett. B215 (1988) 523.

[3] J. Balog, L. Feher, P. Forgacs, L. O'Raifeartaigh and A. Wipf, Phys. Lett. B227 (1989) 214; ibid B244 (1990) 435; Ann. Phys. 203 (1990) 76.

L. Feher, L. O'Raifeartaigh, P. Ruelle, I. Tsutsui and A. Wipf, Ann. Phys. 213 (1992) 1.

L. O'Raifeartaigh, P. Ruelle, I. Tsutsui and A. Wipf, Comm. Math. Phys. 143 (1992) 333.

[4] A.N. Leznov and M.V. Saveliev, Comm. Math. Phys. 89 (1983) 59;

M.V. Saveliev, Comm. Math. Phys. 95 (1984) 199;

A.N. Leznov and M.V. Saveliev, Act. Appl. Math. 16 (1989) 1;

M.V. Saveliev, Mod. Phys. Lett. 5A, 27 (1990) 2223.

[5] F.A. Bais, T. Tjin and P. van Driel, Nucl. Phys. B357 (1991) 632.

[6] P. Bowcock and G.M.T. Watts, preprint EFI 91-63, DTP 91-63.

[7] L. Feher, L. O'Raifeartaigh, P. Ruelle, I. Tsutsui and A. Wipf, Dublin preprint DIAS-STP-91-29

[8] F. Delduc, E. Ragoucy and P. Sorba, Phys. Lett. B279 (1992) 319.

[9] D.A. Leites, M.V. Saveliev and V.V. Serganova, in Proceedings of the Third Yurmale Seminar (VUN Science, Utrecht, The Netherlands, 1986), p. 255.

[10] F. Delduc, E. Ragoucy and P. Sorba, Comm. Math. Phys. 146 (1992) 403.

[11] E. Dynkin, Amer. Math. Soc. Transl. Ser. 2, 6 (1957) 111.

[12] M. Lorente and B. Gruber, J. Math. Phys. 13 (1972) 1639.

[13] V. Drinfeld and V. Sokolov, J. Sov. Math. 30 (1984) 1975.

[14] A.M. Polyakov, Int. Jour. of Mod. Phys. A5(1990) 833.

[15] M. Bershadsky, Comm. Math. Phys. 139 (1991) 71.

[16] E.S. Fradkin and V.Y. Linestsky, preprint ITP-SB-92-34.

[17] R. Slansky, Phys. Rep. 79 (1981) $n^{o} 1$.

[18] L. Frappat, A. Sciarrino and P. Sorba, Comm. Math. Phys. 121 (1989) 457. 
[19] P. Bowcock, preprint EFI-92-09.

[20] M. Ademollo et al. Phys. Lett. B62 (1976) 105.

[21] A. Sevrin, W. Troost and A. Van Proeyen, Phys. Lett. B208 (1988) 447.

[22] P. Goddard and A. Schwimmer, Phys. Lett. B214 (1988) 209.

[23] Yao-Zhong Zhang, preprint BONN-HE-92-05.

F. Toppan, Yao-Zhong Zhang, preprint BONN-HE-92-16.

[24] G.W. Delius, M.T. Grisaru, P. Van Nieuwenhuizen, preprint CERNTH.6458/92.

[25] S. Penati, D. Zanon, preprint IFUM 421/FT.

[26] K. Ito, J.O. Madsen, J.L. Petersen, preprint NBI-HE-92-42. 